\title{
Dependence of dark spot growth on cathode/organic interfacial adhesion in Organic Light Emitting Devices
}

by

Radhika Phatak

\author{
A thesis \\ presented to the University of Waterloo \\ in fulfillment of the \\ thesis requirement for the degree of \\ Master of Applied Science \\ in
}

Electrical and Computer Engineering

Waterloo, Ontario, Canada, 2011

CRadhika Phatak 2011 


\section{Author's Declaration}

I hereby declare that I am the sole author of this thesis. This is a true copy of the thesis, including any required final revisions, as accepted by my examiners. I understand that my thesis may be made electronically available to the public. 


\begin{abstract}
Organic Light Emitting Devices(OLEDs) have several advantages over traditional semiconductor devices such as the possibility of being printable, the potential for low cost fabrication, and the potential ease of integration onto a flexible substrate. However, mass commercialization for OLEDs faces several hurdles including that of ambient stability. Due to issues such as low work function of the cathode in the OLEDs, exposure to oxygen or humidity leads to dark spot formation which detrimentally affects device performance. Protection of OLEDs from ambient conditions places high importance on strict encapsulation techniques. Rigid encapsulation techniques which provide high impermeability to ambient conditions are not easily applicable for flexible OLEDs. Flex-integrable thin film encapsulations do not possess the high integrity of rigid encapsulation structures. If the demand for strict thin film encapsulation could be eased via stronger inherent resistance to dark spot growth, it can be most beneficial for the future of OLEDs, especially in the flexible arena.
\end{abstract}

To ease the need for stringent encapsulation, alternate non-encapsulation related approaches for dark spot suppression are investigated. These include heating the substrate to an elevated temperature during cathode deposition and the use of cathode-organic interfacial layers in the OLED. Observations from microscopy, electrical measurements, surface morphology measurements, and adhesion tests are analyzed. Conclusions on the suppression of dark spot growth are discussed. The role of interfacial adhesion in dark spot suppression is investigated. Ambient stability of OLEDs is shown to be related to stronger interfacial adhesion between the cathode and the organic layers.

A few thin-film encapsulation structures for OLEDs using materials like titanium (Ti) and $\mathrm{MoO} 3$ are also discussed along with the results.

Though dark spot eradication via encapsulation may still prove challenging, dark spot suppression via cathode-organic interfacial adhesion strengthening can be shown to be a promising tentative solution to improve OLED lifetime yield. 


\section{Acknowledgements}

I am greatly indebted to my supervisor, Professor Hany Aziz, for all the wisdom and guidance provided to me during the last two years, while I completed my M.A.Sc.

I am grateful to Professor Ting Tsui for the opportunity to use the adhesion testing setup in his lab.

I would also like to thank Professor Ting Tsui and Professor Yuning Li for taking their time to review my thesis. 


\section{Table of Contents}

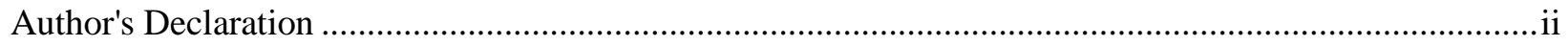

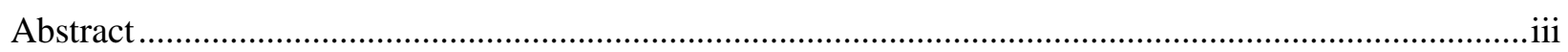

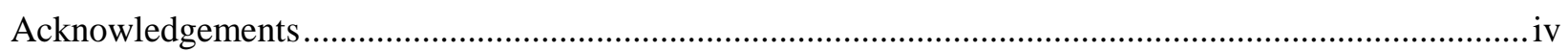

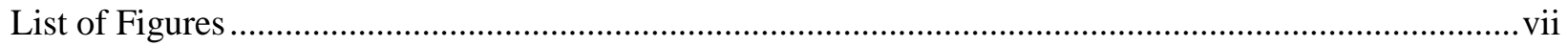

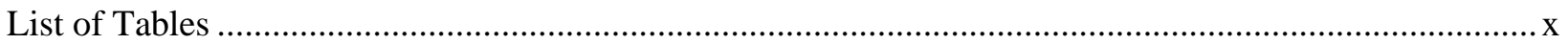

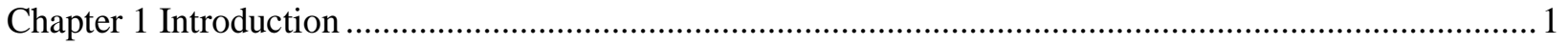

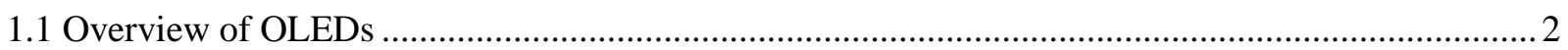

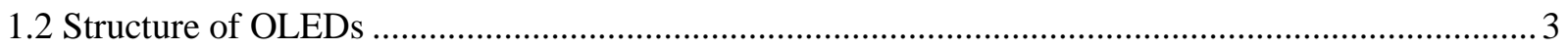

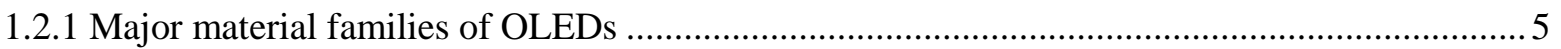

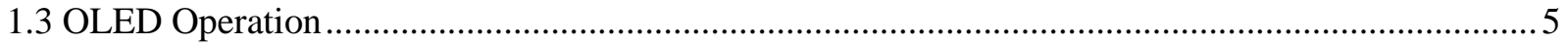

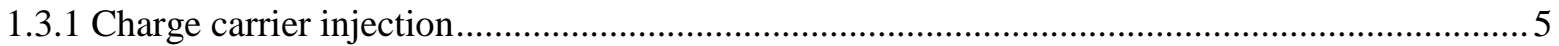

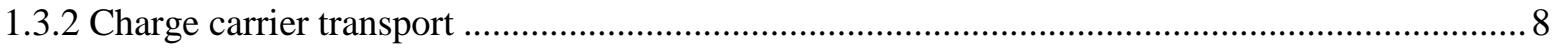

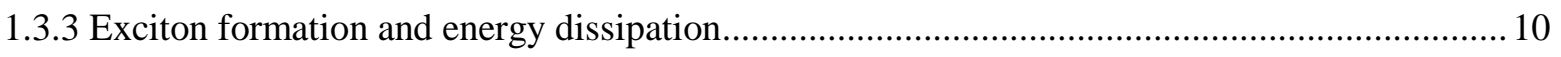

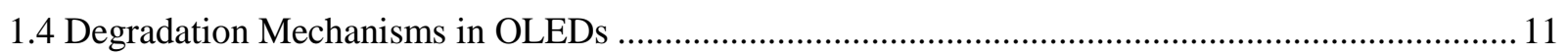

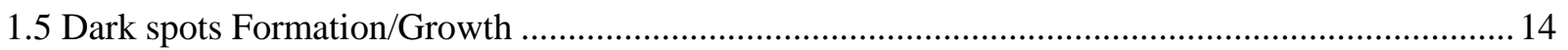

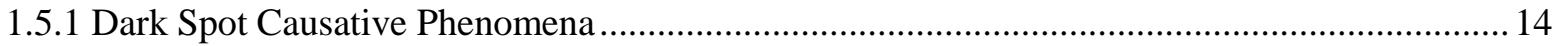

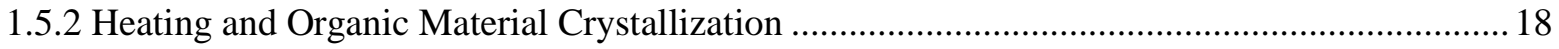

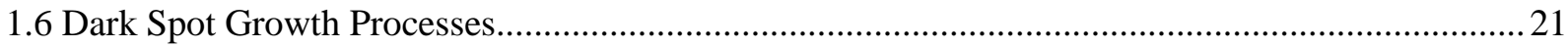

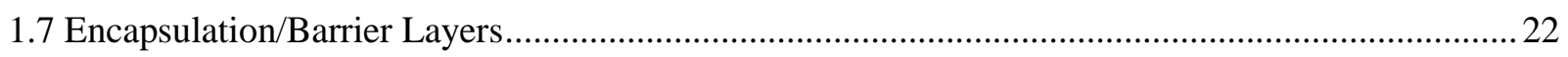

1.7.1 Barix Encapsulation Technology-Thin Film Barrier Layers.................................................. 24

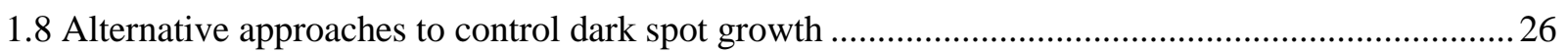

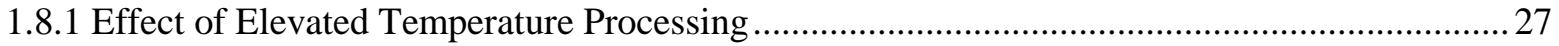

1.8.2 Effect of Cathode-organic interfacial Mixed Organic Metal Layer ......................................... 32

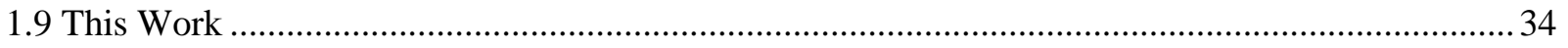

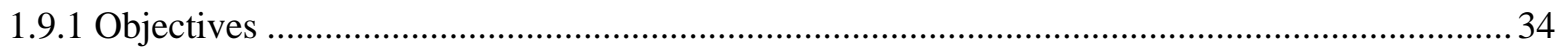

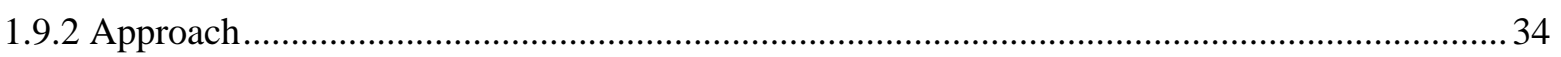

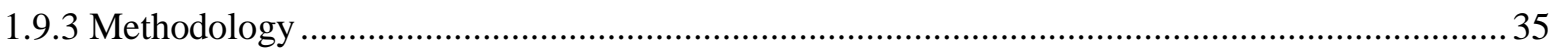

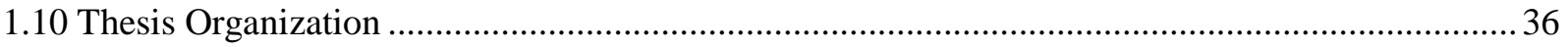

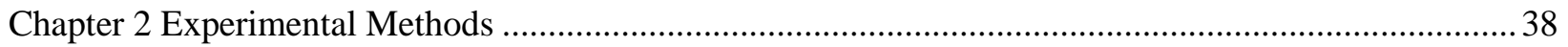

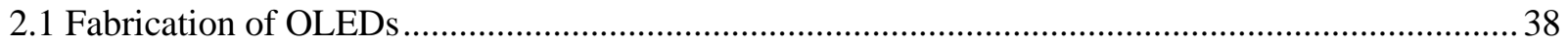

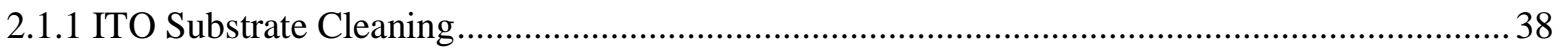

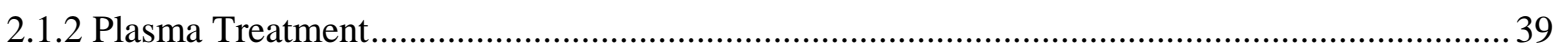




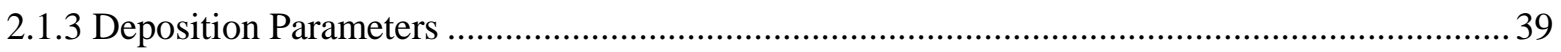

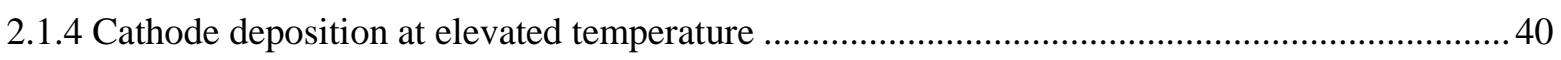

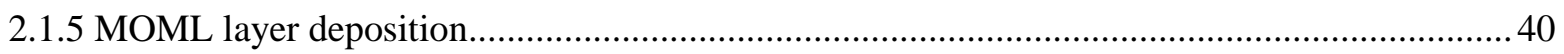

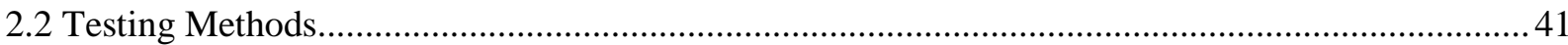

Chapter 3 Effect of cathode deposition temperature on dark spot growth and cathode-organic interfacial

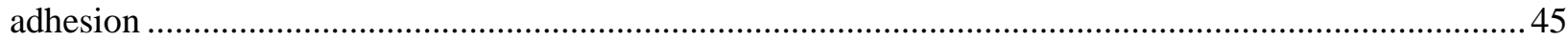

3.1 Effect of elevated temperature cathode deposition on dark spot growth .....................................46

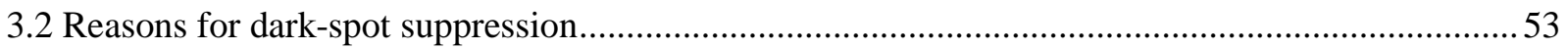

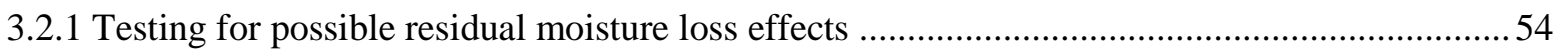

3.2.2 Testing for possible heat induced bare-ITO morphological changes......................................54

3.2.3 Testing for possible morphological changes in the cathode, and at the cathode/organic interface

3.3 Adhesion forces at the cathode-organic interface …....................................................................5

Chapter 4 Effect of using cathode-organic interfacial layers on dark spot growth and cathode-organic

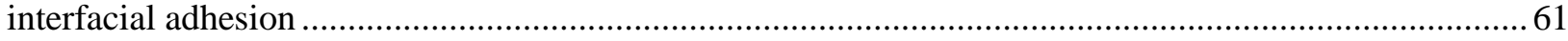

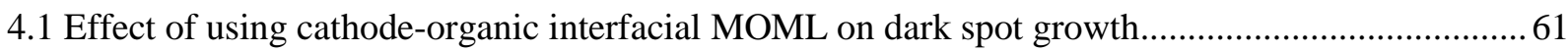

4.2 Effect of using cathode-organic interfacial MOML on cathode-organic interfacial adhesion..........64

4.3 Effect of using abrupt vs. seamless MOML-cathode transition on dark spot growth......................66

4.4 Effect of using $65^{\circ} \mathrm{C}$ deposited MOML $+65^{\circ} \mathrm{C}$ deposited cathode on dark spot growth.................69

4.5 Effect of using electron injection layers on cathode-organic interfacial adhesion.......................... 71

Chapter 5 Exploring novel thin-film encapsulation layers .................................................................... 73

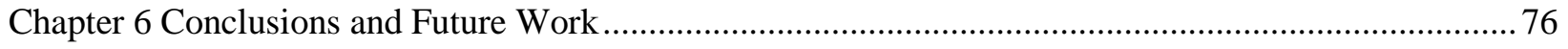

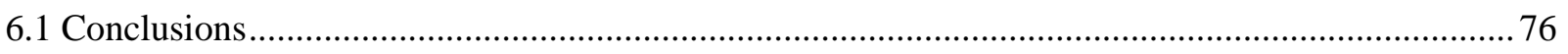

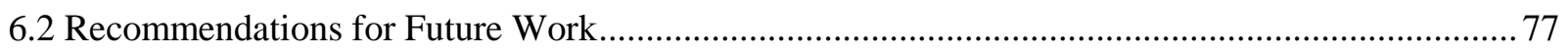

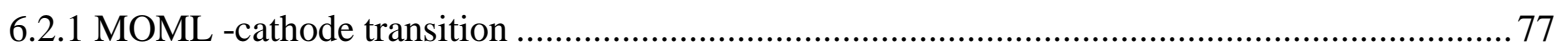

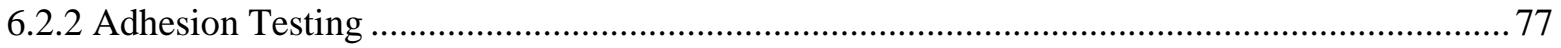

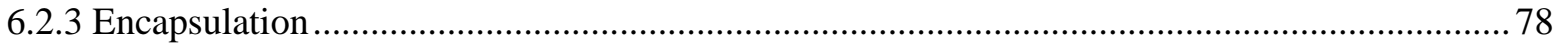

References 


\section{List of Figures}

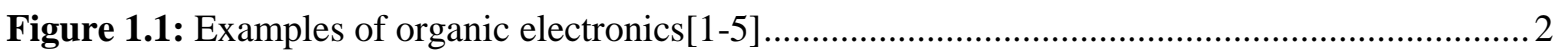

Figure 1.2: Typical structure of single and bi-layer OLEDs........................................................... 3

Figure 1.3: Energy level diagrams for single layer and bi-layer hetero-junction OLEDs[19]. (This figure shows Aluminum as cathode and Indium-tin oxide as anode) ....................................... 4

Figure 1.4: Isolated metal and semiconductor energy band diagram[22] ................................... 6

Figure 1.5: Energy band diagram when metal and semiconductor are brought in contact[22]......... 6

Figure 1.6: Single-layer OLED energy level diagrams[24] ................................................... 7

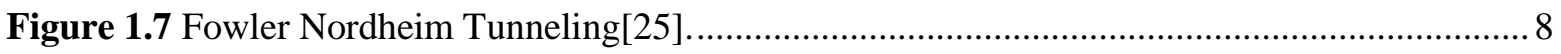

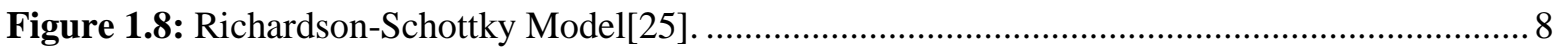

Figure 1.9: Hopping transport(left) vs. Band transport(right). LUMO: Lowest unoccupied molecular orbital, HOMO: Highest occupied molecular orbital[24] ....................................

Figure 1.10: Schematic description of the exciton (left) and singlet and triplet spin descriptions

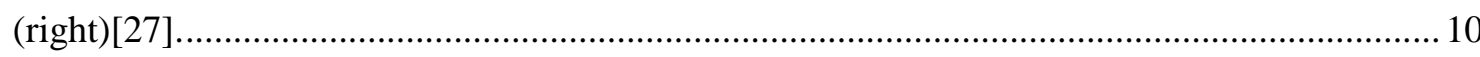

Figure 1.11: Jablonski diagram demonstrating exciton energy dissipation phenomena................... 11

Figure 1.12: Typical dark spot growth after $0 \mathrm{hr}(\mathrm{a})$, and $2 \mathrm{hr}(\mathrm{b})$ exposure to $100 \% \mathrm{RH}$ for a OLED with structure: ITO/NPB/Alq $3 / \mathrm{Mg}: \mathrm{Ag} / \mathrm{Ag}$. The circular rings observed in the bottom part of the images are the magnifying focus of the microscope and can be ignored.

Figure 1.13: Intrinsic degradation - Typical luminance decay $\left(\mathrm{L} / \mathrm{L}_{\mathrm{o}}\right)$ over time for OLEDs. This particular image from literature[29], displays OLEDs with different hole transport layers(HTL).

Figure 1.14: Illustration of foreign materials or irregular "chunks" on the OLED substrate/organic layers and the moisture/oxygen pathways which are created (indicated with arrows)[30]......... 15

Figure 1.15: Bubble like formations labeled 1,2,3 as seen on the OLED emissive surface[35]....... 16

Figure 1.16: Formation of dark spots as a result of gas evolution (This figure shows aluminum as the cathode)[38].

Figure 1.17: Transmission polarization micrograph of an $60 \mathrm{~nm} \mathrm{Alq}$ film stored in humid air at $100 \%$ RH for 6 h showing $\mathrm{Alq}_{3}$ crystallization[39].

Figure 1.18: $\mathrm{AFM}$ scans of $\mathrm{Alq}_{3}$ layer (a) without heat treatment, (b)deposited at $65^{\circ} \mathrm{C}$, (c)annealed at $65^{\circ} \mathrm{C}$. The $\mathrm{Alq}_{3}$ layer here is deposited on top of a pre-existing room temperature deposited NPB layer[45].(Reprinted with permission of Elsevier).

Figure 1.19: Dark spot and bright spots seen after cathode peel off and re-deposition[46].(Reprinted with permission of AIP). .22 
Figure 1.20: Traditional glass/metal rigid encapsulation[47].................................................23

Figure 1.21: WVTR/OTR requirements for various electronics.................................................24

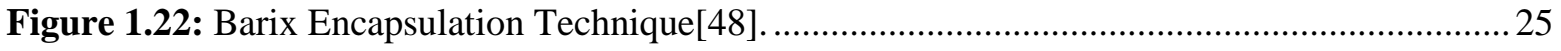

Figure 1.23: Dark spot growth at $0,120,1200$ mins exposure to $40 \% \mathrm{RH}$.Device $\mathrm{A}$ is not heated treated, Device C has its HTL and ETL deposited at $160^{\circ} \mathrm{C}[41]$.(Reprinted with permission of

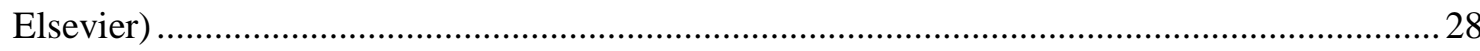

Figure 1.24: Current efficiency and Luminance characteristics for OLEDs with various layers being annealed . The general OLED structure is: $\mathrm{ITO} / \mathrm{NPB} / \mathrm{Alq}_{3} / \mathrm{LiF} / \mathrm{Al}[50]$. (Reprinted with permission of Elsevier) 30

Figure 1.25: Dark spot growth at $0,120,1200$ mins exposure to $40 \% \mathrm{RH}$. The heated device is subject to a temperature of $70^{\circ} \mathrm{C}[52]$. (Reprinted with permission of Elsevier). 31

Figure 1.26: Device A shows the structure of an OLED with a MOML cathode-organic interfacial layer. Device B represents the general non-MOML OLED structure[53]..... 32

Figure 1.27: Dark spot growth after 24 hours ambient exposure for an OLED with a MOML layer (left) and an OLED without a MOML layer (right)[53].

Figure 2.1: Patterned ITO substrate.

Figure 2.2: Four point Bend Configuration. $P$ is the total force exerted onto the sample, $l$ is the moment arm between the inner and outer pins.

Figure 2.3: Typical Force vs. Displacement curve. This particular example uses a compromised $\mathrm{Alq}_{3} / \mathrm{Mg}:$ Ag interfacial stack showing a poor $(<1 \mathrm{~N})$ load at the interface.

Figure 3.1: OLED structure of GRP_1 (a) and GRP_2(b) devices. .46

Figure 3.2: Dark spot growth after 2,17,27hrs exposure to $100 \%$ RH conditions for (a) GRP_1 OLEDs, and (b) GRP_2 OLEDs . 48

Figure 3.3: $\Delta \mathrm{V}$ graph showing voltage changes after 2,17,27hrs exposure to $100 \% \mathrm{RH}$ conditions for GRP_2 and GRP_1 devices.

Figure 3.4: Dark spot growth for GRP_2 (a) vs. GRP_3 (b) at 2,17, and 27hours exposure to 100\% $\mathrm{RH}$ conditions.

Figure 3.5: $\Delta \mathrm{V}$ graph showing voltage changes after 2,17,27hrs exposure to $100 \% \mathrm{RH}$ conditions for GRP_2 and GRP_3 devices.

Figure 3.6: AFM Scans of the top of the cathode for GRP_1 (RT cathode) and GRP_2 $\left(65^{\circ} \mathrm{C}\right.$ deposited cathode) devices...... .56

Figure 3.7: AFM Scans of the top of the organic $\left(\mathrm{Alq}_{3}\right)$ layer for GRP_1 (RT cathode) and GRP_2 $\left(65^{\circ} \mathrm{C}\right.$ deposited cathode) devices . .57

Figure 3.8: General structure of $S \_1, S \_2, S \_3$ samples. .58 
Figure 4.1: Structure of an OLED with a cathode-organic interfacial MOML

Figure 4.2: Dark spot growth for GRP_1 (a) vs. GRP_4 (b) at 2,17, and 27hours exposure to $100 \%$ RH conditions.

Figure 4.3: $\Delta \mathrm{V}$ graph showing voltage changes after $2,17,27 \mathrm{hrs}$ exposure to $100 \% \mathrm{RH}$ conditions for GRP_1 and GRP_4 devices. 64

Figure 4.4: S_4 sample structure .65

Figure 4.5: Dark spot growth after 27 hour exposure to 100\% RH conditions for abrupt(GRP_5) vs. seamless MOML $\rightarrow$ cathode transition (GRP_6) OLEDs .67

Figure 4.6: $\Delta \mathrm{V}$ graph for GRP_5 and GRP_6 OLEDs shown in Fig 3.15... .68

Figure 4.7: Dark spot growth for GRP_4 (a) vs. GRP_7(b) at 2,17, and 27hours exposure to $100 \%$ RH conditions. . .70

Figure 4.8: $\mathrm{S} \_5 / 6$ sample structure .72 


\section{List of Tables}

Table 1.1 OLED characteristics for various annealing strategies[50]. (Reprinted with permission of

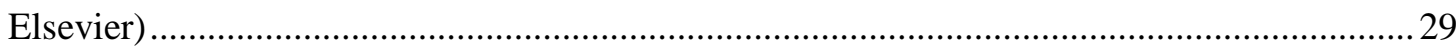

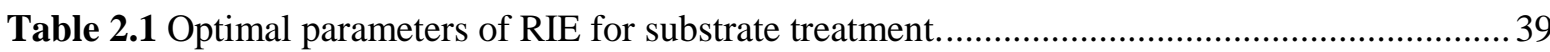

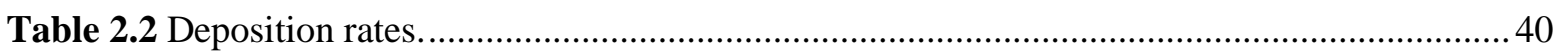

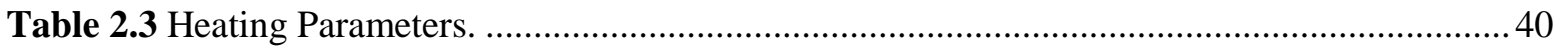

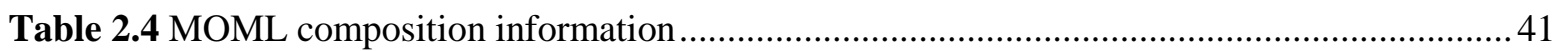

Table 3.1 AFM roughness measurements for the top of the cathode layer .....................................56

Table 3.2 AFM roughness measurements for the top of the organic layer underlying the cathode ...57

Table 3.3 Energy release rates at the cathode-organic interface for $S \_1, S \_2, S \_3$ samples ...............60

Table 4.1 Energy release rates at the cathode-organic interface for $\mathrm{S} \_1$ and $\mathrm{S} \_4$ samples.

Table 4.2 Average $\Delta \mathrm{V}$ after $27 \mathrm{hrs}$ exposure to $100 \% \mathrm{RH}$ conditions for seamless(GRP_6) and

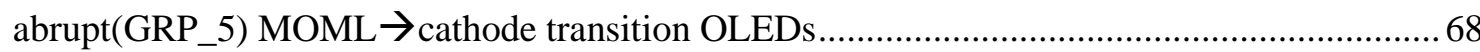

Table 4.3 Energy release rates at the cathode-organic interface for samples $S \_1, S \_5$, and S_6 ....... 72

Table 5.1 Average brightness and voltage measurements for OLEDs with a varying cathode thicknesses. The titanium layer thickness is maintained at $200 \mathrm{~nm}$.

Table 5.2 Average brightness and voltage measurements for OLEDs with a varying titanium thicknesses. The Mg:Ag cathode layer thickness is maintained at $100 \mathrm{~nm}$. 


\section{Chapter 1Introduction}

Organic electronics seem to be gaining popularity in today's market due to their salient advantages such as potential of lower cost manufacturing, low power consumption ,ecofriendliness. Some of the other advantages of organic electronics over conventional electronics are that they can be printable and coatable and have the potential to be used in flexible electronics.

The organic electronic applications are widespread and they can be seen in Charge Transport systems like transistors and diodes (organic active matrix backplanes for displays, printed integrated circuits, etc.), in electroluminescent systems like Polymer Light Emitting Devices, Organic Light Emitting Devices (OLEDs) (full color active matrix displays, large area uniform displays, etc.), and in Photovoltaic cells (battery chargers for mobile devices, smart packaging, traffic signs etc). Figure 1.1 shows several applications of organic electronics[1-5].

OLEDs have entered the commercial market in devices such as displays and solid state lighting systems and possess very promising properties such as faster refresh rates, wider viewing angles, and better color contrast. OLEDs are forecasted to account for US $\$ 5.6 \mathrm{~B}$ in the organic electronics market by 2015[6]. However to overcome poor ambient stability, attributed to moisture/oxygen susceptible low work function cathode and active organic layers, OLEDs usually utilize high integrity encapsulation for commercial viability. Although rigid encapsulation has become ubiquitous for non-flexible OLEDs, encapsulation of flexible OLEDs still presents a challenge. Thin film encapsulation might provide a solution for flexible OLEDs, but it lacks the high integrity moisture/oxygen impermeability as seen in glass/rigid barriers. Hence, a need exists to strengthen the inherent ambient stability of OLEDs to ease the need for stringent encapsulation in the flexible arena. 


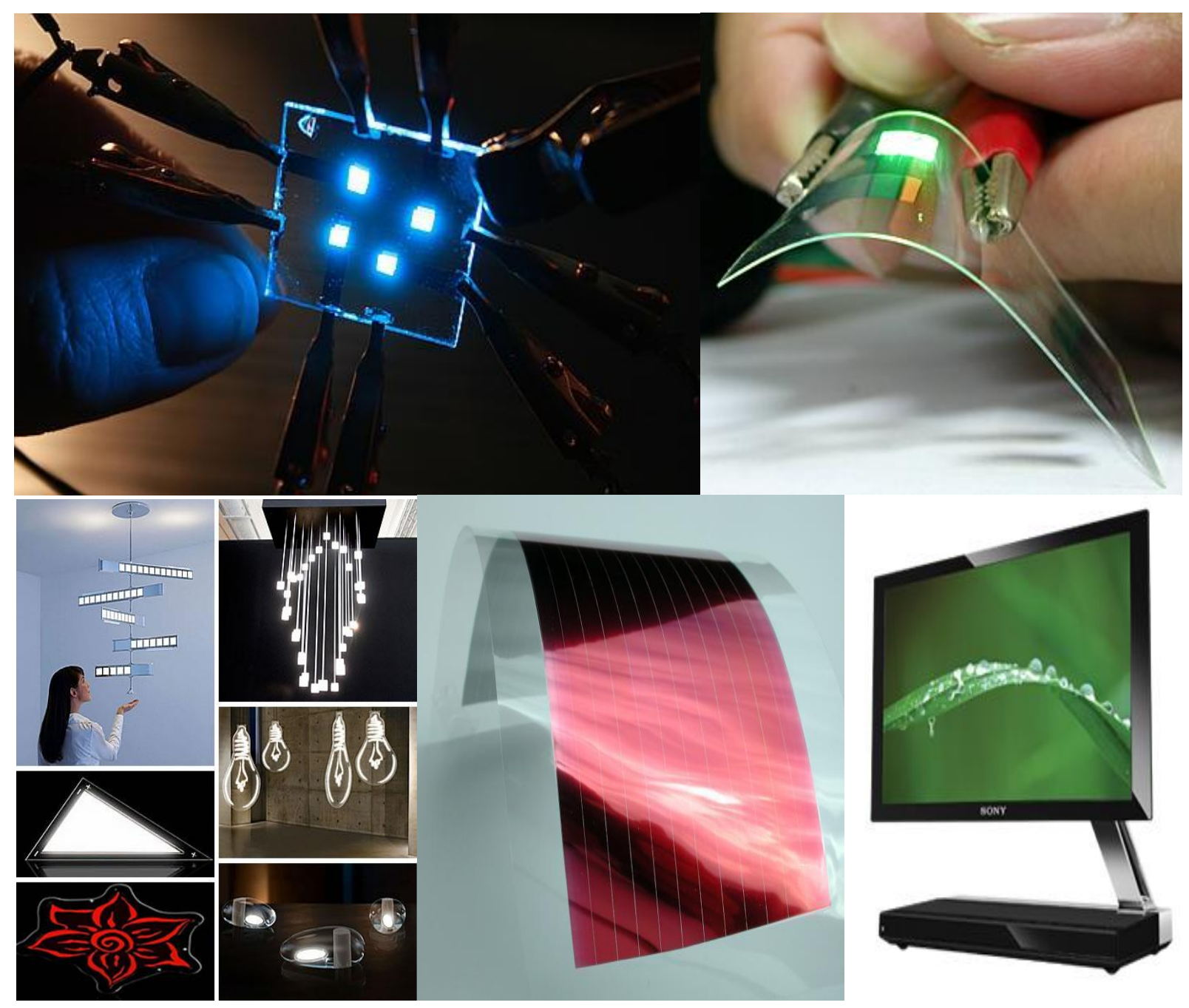

Figure 1.1: Examples of organic electronics[1-5]

\subsection{Overview of OLEDs}

OLEDs emit light based on the phenomena of electroluminescence(EL). When supplied with external power, OLEDs can generate light based on the organic light-emitting materials used to fabricate the OLED. Although the concept of electroluminescence in organic materials was realized as early as 1950s, the first OLED diode structure was only reported in 1987[7]. Prior to 1987, electroluminescence was observed from single crystals of aromatic hydrocarbons like anthracene [8](Helfrich \& Scneider in 1965). In 1987 Ching W. Tang and Steven Van Slyke reported the first diode device consisting of a two-layer structure with separate hole transporting 
and electron transporting layers such that recombination and light emission occurred in the middle of the organic layer. This resulted in a reduction in operating voltage and improvements in efficiency [7](when compared to single layer devices) and led to the current era of OLED research and device production.

\subsection{Structure of OLEDs}

A typical structure of an organic light-emitting device consists of one or more organic layers sandwiched between two electrodes which can be either metallic or semi-conducting [59]. The organic layers can act both as emissive layers and as charge transport and/or injection layers to improve the performance of the devices.[9-18]. Fig 1.2 shows two basic OLED configurations: a) single-layer structure, and b) bi-layer structure.

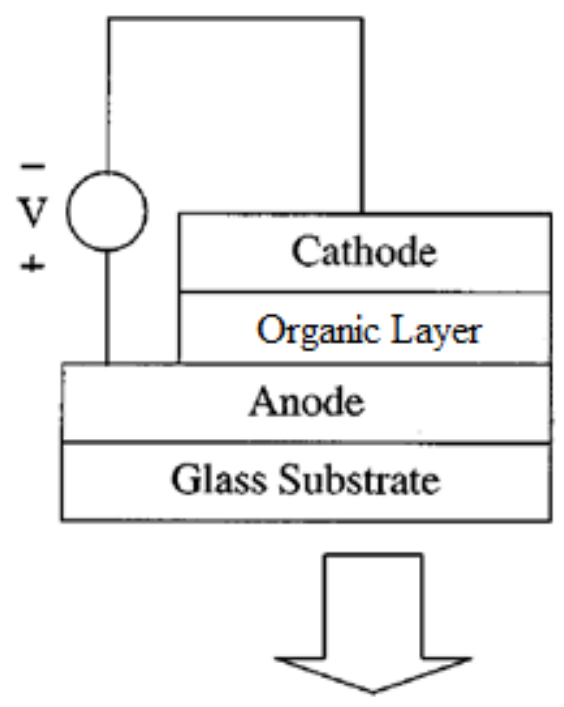

EL Emission

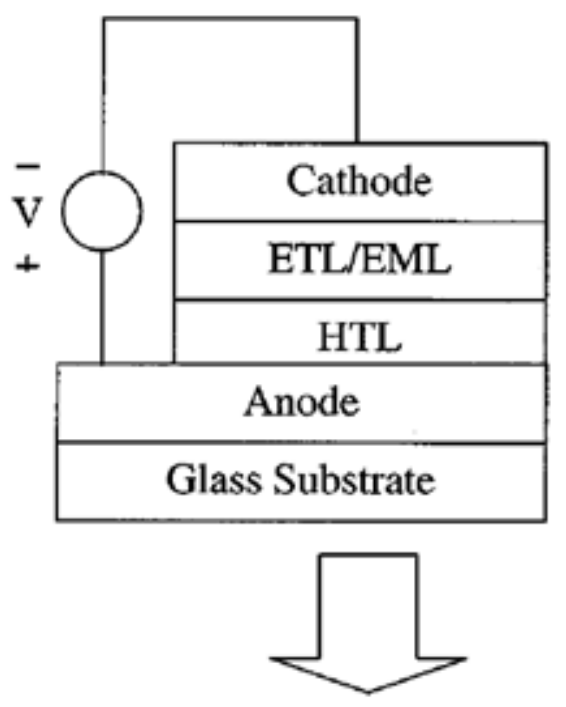

EL Emission

Figure 1.2: Typical structure of single and bi-layer OLEDs.

For a bi-layer heterojunction OLED the device structure consists of a transparent high workfunction anode, a hole transport layer (HTL), a electron transport layer (ETL) and a low work function cathode. When a forward bias is applied to an OLED, holes and electrons are injected 
from anode and cathode, and are transported through HTL and ETL, respectively, recombining to form excitons in the emissive layer (EML). Finally the excitons either decay radiatively to emit photons or dissipate non-radiatively.

On the other hand, for a single layer OLED, the structure consists of just one organic layer sandwiched between two electrodes. When a forward bias is applied to a single-layer OLED, the holes and electrons are injected into the common organic layer which acts both as a transport layer for the charge carriers and also as the site for exciton formation and light emission (Fig 1.3). However, in these types of OLEDs, since charge recombination and light emission happens in the single organic layer, the area near the contacting electrodes can exhibit luminescence quenching which affects overall device performance[19].

\section{Single-Layer OLEDs}

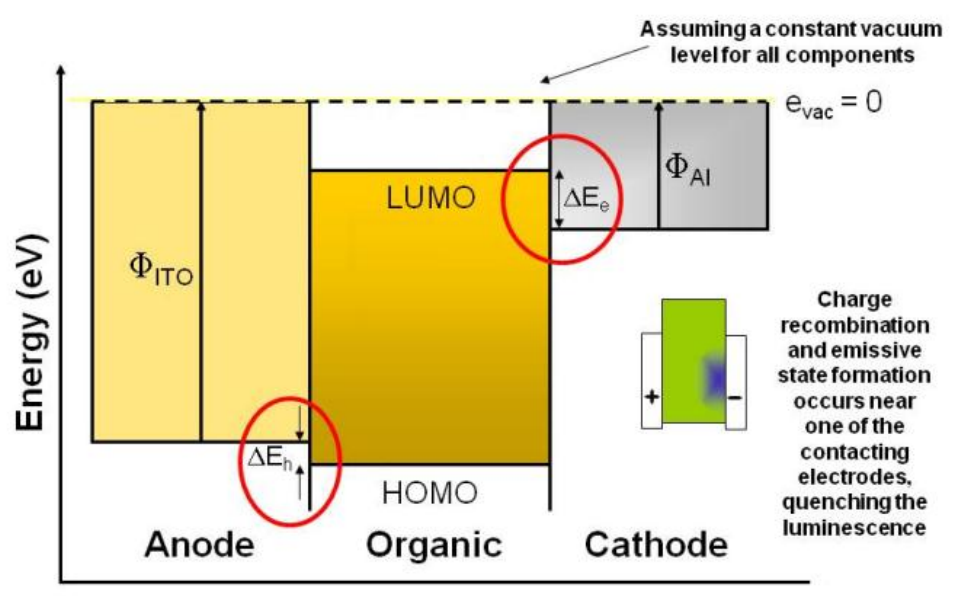

\section{Bi-Layer Heterojunction OLEDs}

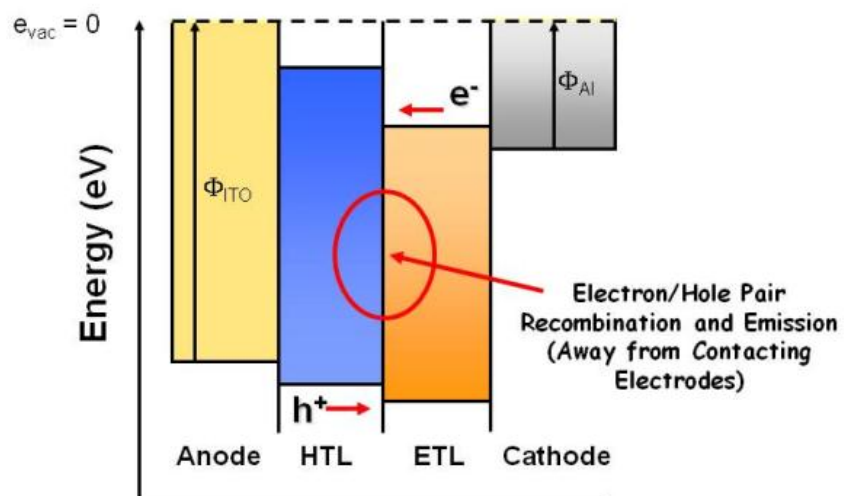

Figure 1.3: Energy level diagrams for single layer and bi-layer hetero-junction OLEDs[19].

(This figure shows Aluminum as cathode and Indium-tin oxide as anode) 


\subsubsection{Major material families of OLEDs}

a) SM-OLEDs: These types of OLEDs utilize small molecule organic materials in between their electrodes, hence giving them the name: small molecule organic light emitting devices (SM-OLEDs). Molecules commonly used in SM-OLEDs include organometallic chelates, fluorescent and phosphorescent dyes and conjugated dendrimers[20]. SM-OLEDs usually employ thermal evaporation procedures for fabrication making the production process more expensive and not as easily applicable for large area devices. However, thermal evaporation yields well controlled, homogeneous films, and due to its high flexibility in layer design, it helps in achieving high efficiencies for the SM-OLEDs[20]. The OLEDs utilized in this thesis are SMOLEDs.

b) PLEDs: Polymer Light Emitting Devices (PLEDs) utilize electroluminescent conductive polymers that emit light when connected to an external voltage. Typical polymers used in PLED displays include derivatives of poly(p-phenylene vinylene) and polyfluorene[20]. PLEDs usually employ spin-coating as the method for depositing thin polymer films. Although spin-coating is promising for large-area devices, the construction of complex multi-layer structures proves difficult with this approach.

\subsection{OLED Operation}

The OLED operational mechanism depends on three processes: charge carrier injection, charge carrier transport and exciton formation and dissipation.

\subsubsection{Charge carrier injection}

Carrier injection takes place at the two electrode-semiconductor interfaces. In the case of metal-semiconductor interfaces, the Mott-Schottky model [21]can provide some insight about carrier injection. Fig 1.4 [22] shows the energy band diagrams of an isolated metal adjacent to an isolated n-type semiconductor. 


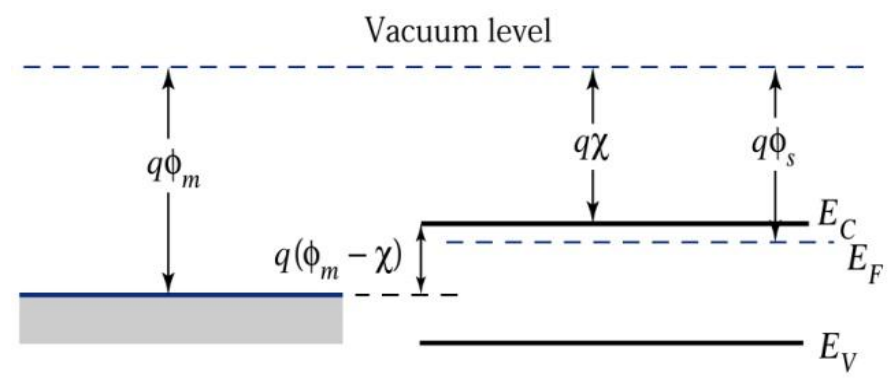

Metal

Semiconductor

Figure 1.4: Isolated metal and semiconductor energy band diagram[22].

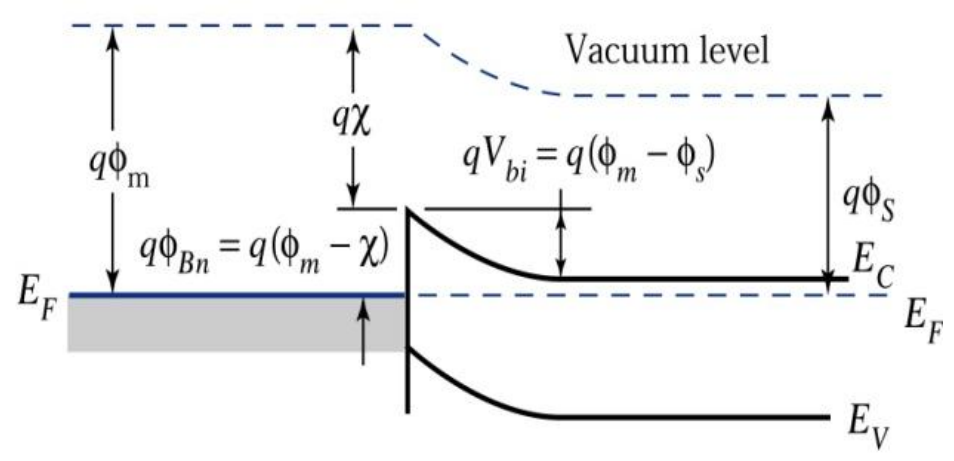

Figure 1.5: Energy band diagram when metal and semiconductor are brought in contact[22].

When the electrodes and semiconductors are brought into contact, it leads to energy level realignment as shown in Fig 1.5[22]. Bringing the metal and semiconductor in contact will lead to flow of electrons (from the side of higher $E_{f}$ (Fermi energy) to the other) until alignment of $E_{f}$ in the two materials is reached. Although recent research has proved that the Fermi energy levels do not perfectly align, and there exists a vacuum level shift $\Delta$ offset value to be considered[23], we will regard the Mott-Schottky model as a simplistic model in understanding energy alignment issues at the metal-semiconductor interface.

In organic electronic devices, band bending is closely related to the potential profile across the organic layers in the devices, with an applied external electric field. In OLEDs, the electric field is often assumed to be constant and the potential is proportional to the position . Hence a simple illustration of energy level changes in OLEDs can be shown in Fig 1.6[24]. As 
seen in the figure, when the OLED is subject to forward bias, the energy barrier for electrons and holes is low, and it aids in easy charge injection at the electrode-semiconductor interface. On the other hand, when the OLED is subject to reverse bias, the energy barrier for carrier injection becomes much higher, leading to inefficient injection at the electrode-semiconductor interfaces. Due to this an insignificant current flow is observed when the OLED is in reverse bias.
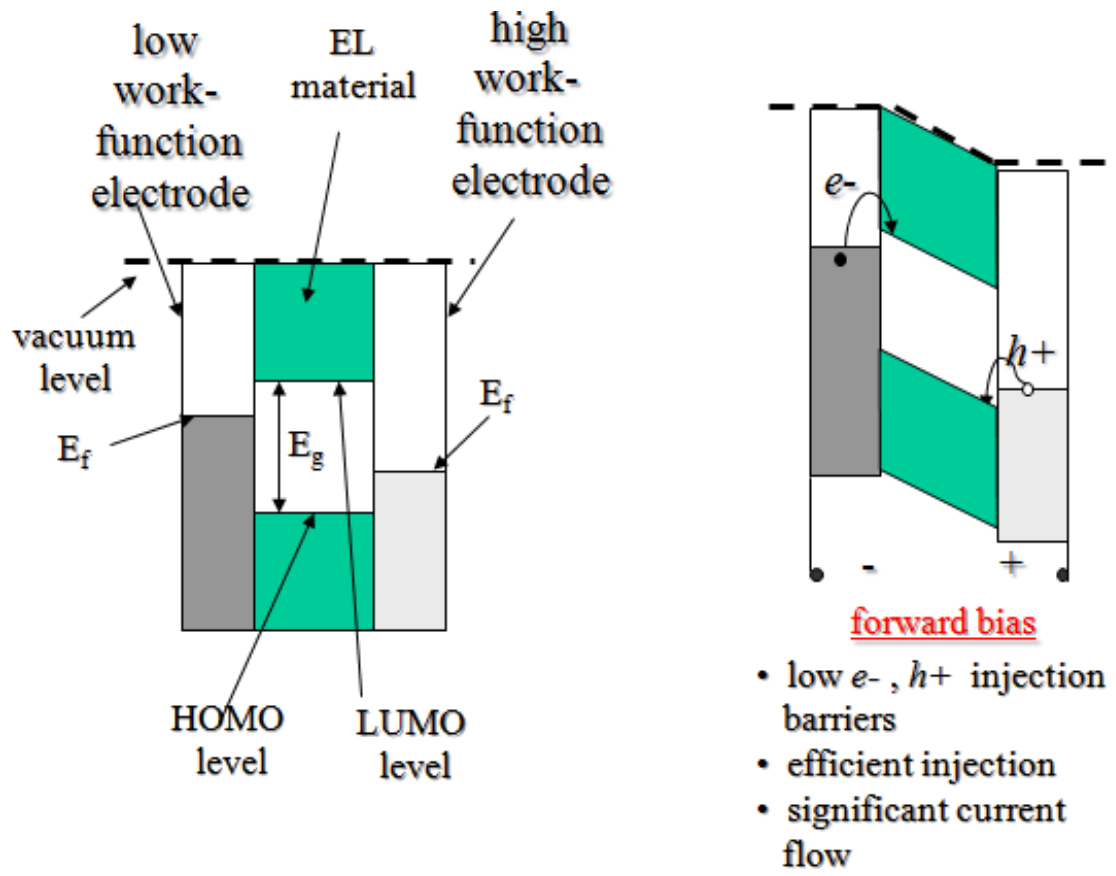

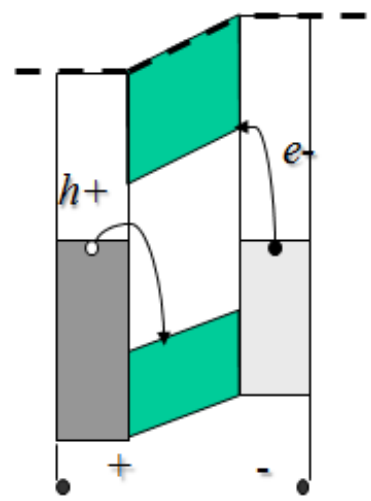

reverse bias

- high $e^{-}, h+$ injection barriers

- inefficient injection

- insignificant current flow

Figure 1.6: Single-layer OLED energy level diagrams[24].

Two models are often used to describe injection of the carriers from the metal electrode into the semiconductor. The first one is called Fowler-Nordheim Model and it explains the direct-tunnelling of charges through the triangular energy barrier as shown in Fig 1.7. The higher the electric field across the interface, the stronger the tunnelling observed. The second model is called the Richardson-Schottky Model (illustrated in Fig 1.8) and it demonstrates the fieldassisted thermionic injection over the image force barrier*. Any deformations at the electrodesemiconductor interface can affect charge injection in an OLED and severely reduce device

\footnotetext{
* Image force potential: An electron at a distance $\mathrm{X}$ from the surface of a metal experiences a force as if there were a positive charge of $+\mathrm{e}$ at a distance of $2 \mathrm{x}$ from it. This force, which tries to prevent the escape of the electron from the metal, gives a potential energy called the image force potential.
} 
efficiency and performance. Hence, it is of utmost importance to maintain the integrity of the electrode-semiconductor interface in order to ensure superior device performance and lifetime.

$$
j_{F N}=\frac{A^{*}}{\Phi_{B}}\left(\frac{q F}{\alpha k_{B}}\right)^{2} \exp \left(-\frac{2 \alpha \Phi_{B}^{3 / 2}}{3 q F}\right)
$$

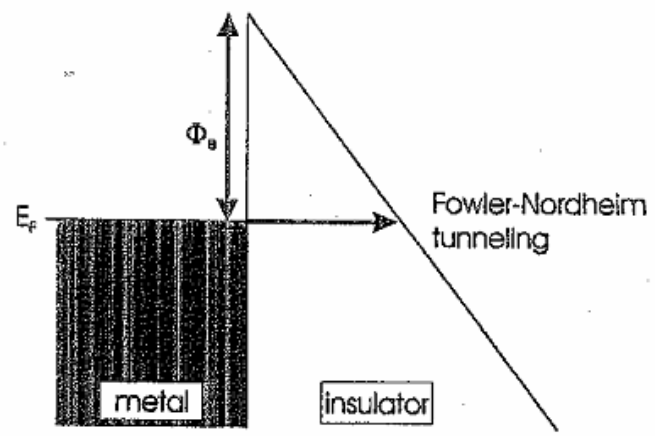

Figure 1.7 Fowler Nordheim Tunneling[25].

$$
j_{R S}=A^{*} T^{2} \exp \left(-\frac{\Phi_{B}-\Delta \Phi}{k_{B} T}\right), \quad \Delta \Phi=\sqrt{\frac{q^{3} \cdot F}{4 \pi \cdot \varepsilon_{r} \cdot \varepsilon_{0}}}
$$

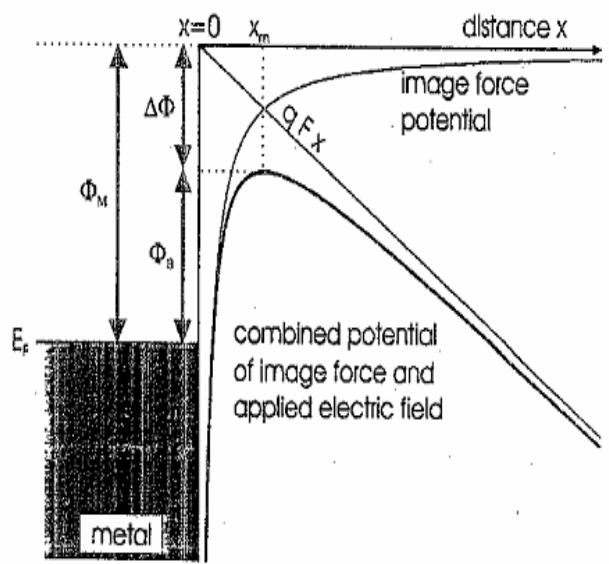

Figure 1.8: Richardson-Schottky Model[25].

\subsubsection{Charge carrier transport}

In conventional inorganic semiconductors, the crystals are bonded together by means of covalent or ionic bonds, and charge carriers in the crystals are delocalized over the crystal lattice. 
In this case, carrier transport is through charges which can move freely in the energy bands. This is called band transport. On the other hand, organic semiconductor materials are bonded together by relatively weak Van der Waals forces and the charge carriers are localized to individual molecular sites. Charge transport in amorphous organic semiconductor materials occurs by hopping through Gaussian distribution of localized states with superimposed potential disorder. The relaxation time of carriers between scattering events is lesser than the carrier residence time on the scattering site - i.e. the carriers are not as mobile as seen in band transport[24].

The following equation [24]represents the mobility as a result of hopping transport seen in organic semiconductor materials:

$$
\mu \propto \mu_{0} \exp \left[-\left(\frac{2 \sigma}{3}\right)^{2}\right] \exp \left[C\left(\sigma^{2}-\Sigma^{2}\right) \sqrt{F}\right]
$$

where $\sigma=$ represents Density of States (DOS), C= constant, $\Sigma=$ represents positional disorder, $F=$ represents electric field

From this equation we can see that hopping transport in organic semiconductor materials is dependent on electric field strength. Fig 1.9[24] shows a diagram of hopping transport vs. band transport.
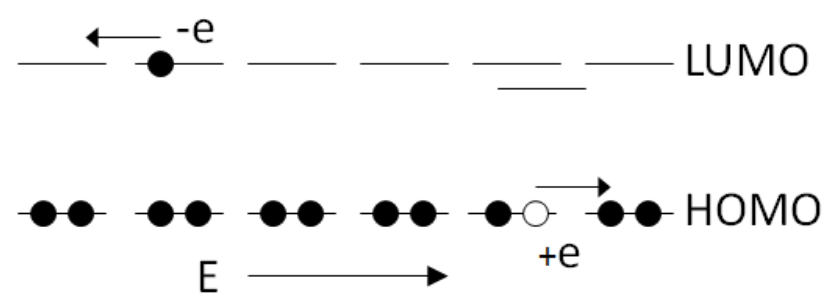

Hopping Transport
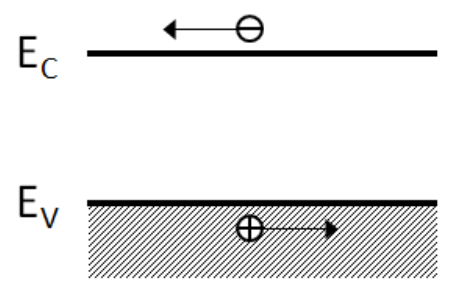

Band Transport

Figure 1.9: Hopping transport(left) vs. Band transport(right). LUMO: Lowest unoccupied molecular orbital, HOMO: Highest occupied molecular orbital[24]. 


\subsubsection{Exciton formation and energy dissipation}

The charge carrier, once transported into the emissive layer of the OLED, will recombine to form excitons. An exciton is a bound state of an electron and hole, in a non-ground energy level. The electron and the hole are attracted to each other by the electrostatic Coulomb force[26]. Depending on the degree of delocalization of the electron around the hole, excitons can be classified as a)Frenkel excitons(highly localized), Wannier-Mott excitons(weakly localized), and Charge-transfer excitons(intermediate degree of localization).

Excitons can also be categorized as being in singlet or triplet state, based on the spin configuration of the electrons in the highest occupied molecular orbital (HOMO) and the lowest unoccupied molecular orbital (LUMO). If the two unpaired electrons have the same spin, the exciton can be considered to be in triplet state. However, if the spins of the two unpaired electrons are opposite, it is considered a singlet.

As can be seen from Fig 1.10[27], three out of the four possible spin state configurations lead to the creation of triplets. Hence there is a $75 \%$ chance that excitons are in a triplet state rather than in a singlet state $(25 \%$ chance).
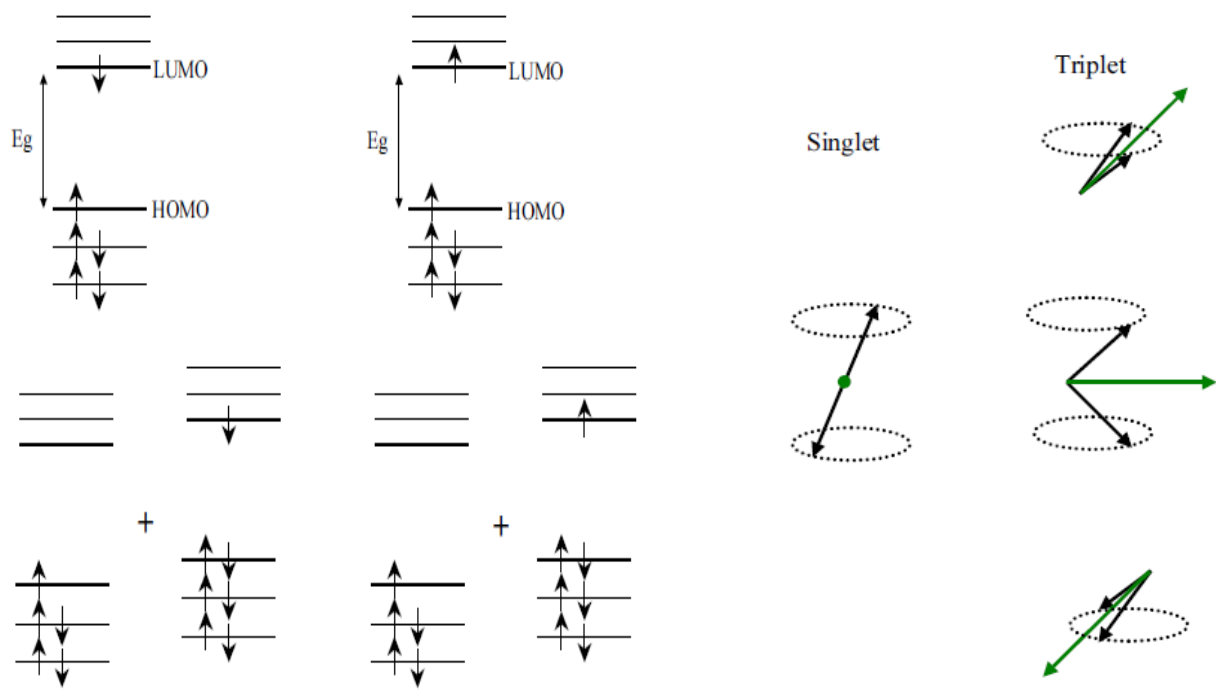

Figure 1.10: Schematic description of the exciton (left) and singlet and triplet spin descriptions (right)[27]. 
The energy dissipation of an exciton can take two routes: radiative transition or radiationless transition [27]. Phenomena such a fluorescence, delayed fluorescence, and phosphorescence are categorized as radiative transitions. Internal conversion and intersystem crossing are classified as radiation-less transitions. Fig 1.11 shows a Jablonski diagram demonstrating the aforementioned dissipation phenomena.

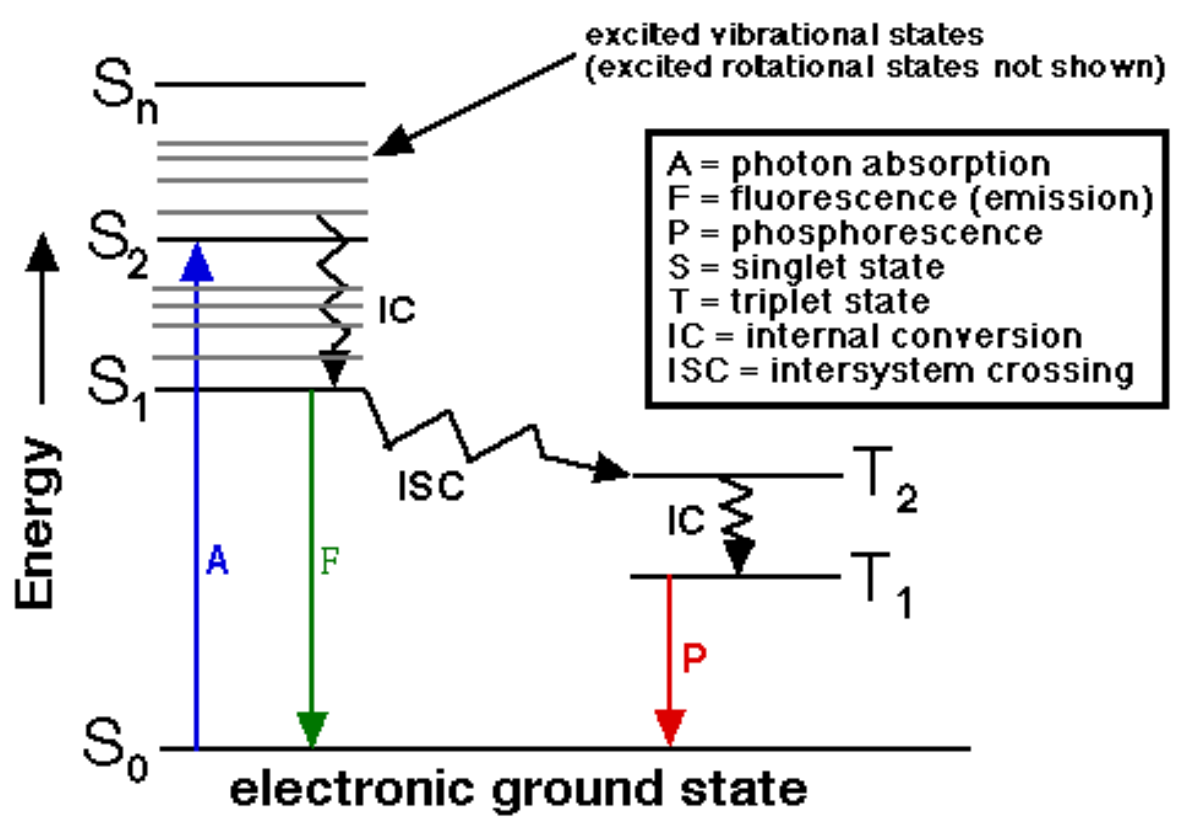

Figure 1.11: Jablonski diagram demonstrating exciton energy dissipation phenomena

\subsection{Degradation Mechanisms in OLEDs}

Degradation of an OLED's performance over time can be attributed to three major categories[28]. These categories, which are listed below, detrimentally affect the device luminance and thereby diminish device integrity.

1) Dark-spot degradation: Dark-spot degradation refers to OLED degradation as a result of formation of non-emissive dark spots within the emissive area of the device. These nonemissive areas result in the decrease of total device luminance and lead to device degradation. Dark spot degradation is accelerated by the presence of moisture and oxygen, leading to severe device degradation. This type of degradation can be controlled 
via device encapsulation. However encapsulation of flexible OLEDs still poses a challenge as rigid encapsulation cannot be utilized, and existing thin film encapsulation techniques lack the high impermeability of glass encapsulation. Due to the lack of suitable flexible encapsulation, dark spot degradation can be considered as a major mode of degradation in flexible OLEDs. Fig 1.12 shows typical dark spot growth after 0 and 2 hours exposure to $100 \% \mathrm{RH}$ in air, for an unencapsulated OLED. Various causes for dark spots formation and growth have been proposed, and are discussed in depth in section 1.5 below.

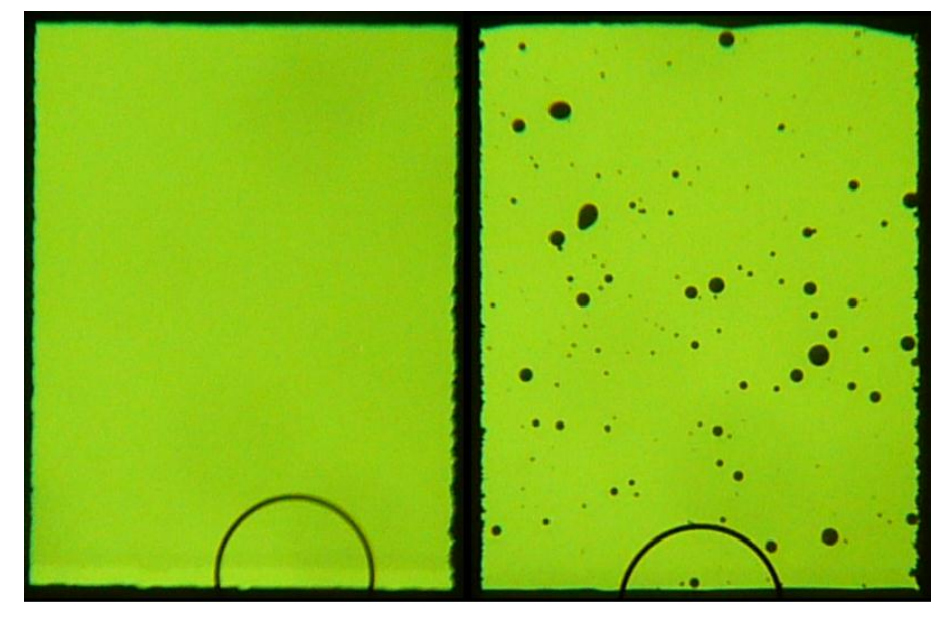

a)

b)

Figure 1.12: Typical dark spot growth after $0 \mathrm{hr}(\mathrm{a})$, and $2 \mathrm{hr}(\mathrm{b})$ exposure to $100 \% \mathrm{RH}$ for a OLED with structure: ITO/NPB/Alq $3 / \mathrm{Mg}: \mathrm{Ag} / \mathrm{Ag}^{\varpi}$. The circular rings observed in the bottom part of the images are the magnifying focus of the microscope and can be ignored.

2) Catastrophic degradation: Catastrophic degradation refers to a sudden decrease or total loss of OLED luminance due to the development of electrical shorts across the device[28]. These shorts result from pre-existing morphological defects in the organic layers or the electrodes of the OLED. This type of degradation can be easily controlled by using materials with high morphological integrity and by utilizing deposition techniques that yield uniform films with minimal defects.

\footnotetext{
${ }^{\varpi} \mathrm{NPB}: \mathrm{N}, \mathrm{N}^{\prime}$-di(naphthalene-1-yl)-N,N'-diphenyl-benzidine $\mathrm{AlQ}_{3}$ : $\operatorname{Tris}(8$-hydroxyquinoline) aluminum
} 
3) Intrinsic degradation: Intrinsic degradation refers to the progressive decrease in the luminance of the OLED in time that occurs during device operation[28]. This type of degradation leads to an intrinsic decrease in the electroluminescence quantum efficiency of the OLED. Fig 1.13 shows the typical progressive decrease of luminance over device operation time for OLEDs as a result of intrinsic degradation[29].

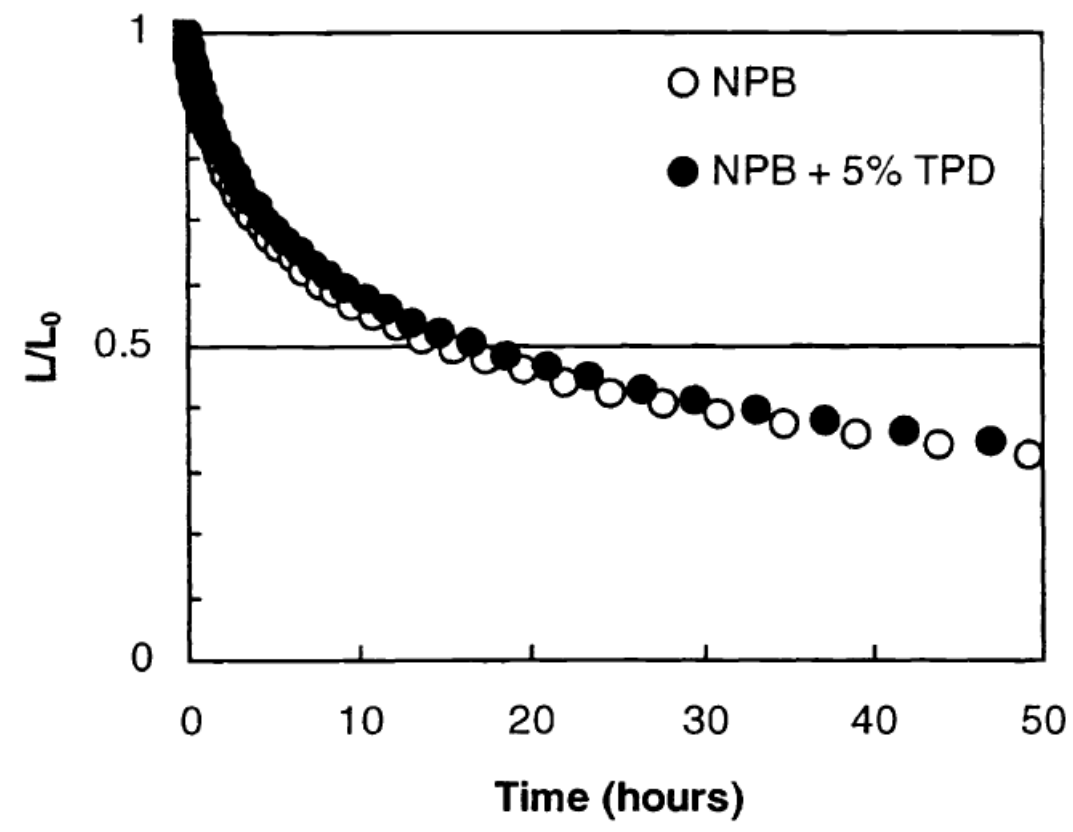

Figure 1.13: Intrinsic degradation - Typical luminance decay $\left(\mathrm{L} / \mathrm{L}_{\mathrm{o}}\right)$ over time for OLEDs. This particular image from literature[29], displays OLEDs with different hole transport layers(HTL).

The following are some of the models proposed to explain intrinsic degradation[28]:

i. Morphological instability model

ii. Unstable cationic $\mathrm{Alq}_{3}$ model

iii. Indium migration model

iv. Mobile ionic impurities model

v. Immobile positive charge accumulation model 
It is important to note that this thesis study is directed towards improving OLED ambient stability via controlling ambient degradation for flexible OLEDs. Due to the lack of high efficiency, easily integrable encapsulation for flexible OLEDs, dark spot degradation is the most relevant mode of device degradation in this work.

\subsection{Dark spots Formation/Growth}

Dark spot degradation is caused by the evolution of structural defects in the OLED layers, and more specifically at the two electrode/organic interfaces but to a larger extent at the cathode/organic interface[28].

\subsubsection{Dark Spot Causative Phenomena}

Dark spots can be attributed to the following causative phenomena in an OLED:

1. Foreign materials lodged onto the substrate or onto the organic layers while deposition lead to direct pathways for organic layer and cathode-organic interfacial compromise by oxygen/moisture(Fig 1.14). Fig. 1.14( a) shows particle or an asperity that pre-exists on the surface of ITO/glass before the organic deposition[30]. Also shown in Fig 1.14(b) is an organic "chunk" that might be deposited by "spitting'" of the organic material during organic deposition[30]. These foreign particles may be larger in size than the thickness of the organic layers. This may lead to incomplete coverage by organic deposition leading to clear conduits for moisture/oxygen propagation. Hence cleanliness of the substrate and fabrication chamber is of utmost importance in order to reduce dark spots. 


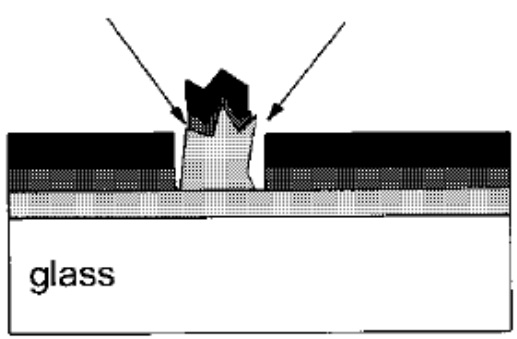

(a)

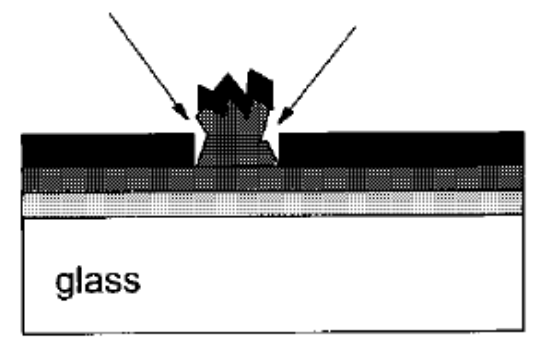

(b)

\section{ITO \\ organic \\ $\mathrm{Mg} / \mathrm{Ag}$}

Figure 1.14: Illustration of foreign materials or irregular "chunks" on the OLED substrate/organic layers and the moisture/oxygen pathways which are created (indicated with arrows)[30].

2. Cathode Pinholes: pinholes are minute pathways created in a deposited layer which result due to uneven layer deposition. Pinholes created in the cathode and/or protective layers on top of the cathode provide pathways for oxygen and/or moisture to penetrate through the sensitive metal layers(low work function- prone to oxidation) and reach the susceptible active-organic layers. The result of this diffusion is device compromise. Oxidation of the cathode can lead to inhibited electron injection at the cathode-organic interface, and the compromise of the organic layers leads to device performance degradation. Studies on the growth rate of dark spots have shown that the growth rate is governed by pinhole size[31]. The larger the pinhole, the faster the growth rate- leading to short device lifetimes[31]. Hence, the need for extremely uniform deposition of cathode layers is crucial for improving device lifetimes. 
3. "Bubble"-formation/gas evolution: Gas evolution produced by electrochemical processes at the cathode/organic interface in the presence of ambient moisture/oxygen leads to the formation of bubble like structures at the cathode/organic interface. These dome-like or bubble-like structures lead to cathode delamination from the organic layers underneath[32-37]. This negatively affects electron injection at the cathode-organic interface and dark spots are observed at the site of contact loss. Figures 1.15 and1.16 show the bubble formation as a result of gas evolution at the cathode-organic interface

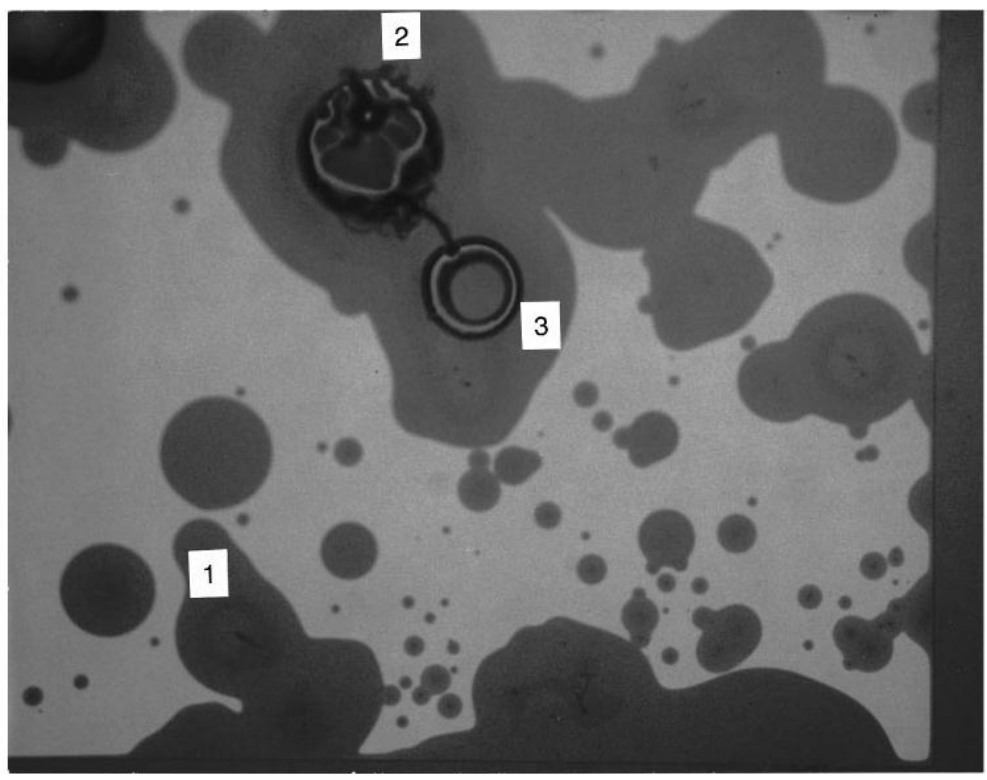

Figure 1.15: Bubble like formations labeled 1,2,3 as seen on the OLED emissive surface[35]. 

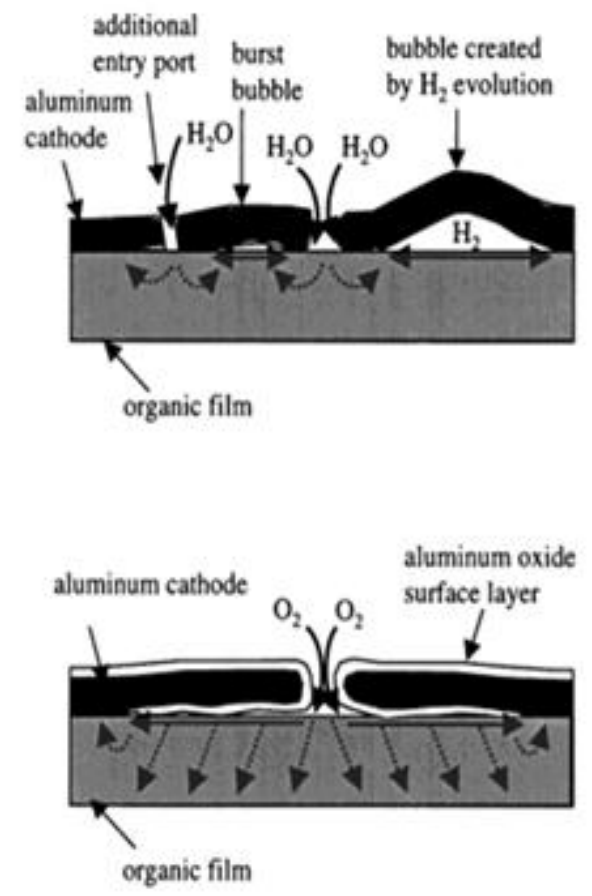

Figure 1.16: Formation of dark spots as a result of gas evolution (This figure shows aluminum as the cathode)[38].

4. $\mathrm{Alq}_{3}$ crystallization: Aziz et al. reported that exposure to humidity(propagated through pinholes) induces the formation of $\mathrm{Alq}_{3}$ crystallites in originally amorphous $\mathrm{Alq}_{3}$ films[39]. These crystals form protruding lumps that are several times thicker than the original film, and have a water content higher than that in the amorphous $\mathrm{Alq}_{3}$ regions[39]. The diffusion of water into the device through microscopic defects in the cathode may lead to crystallization of $\mathrm{Alq}_{3}$ in the OLED structure itself, leading to growth of $\mathrm{Alq}_{3}$ crystals. These crystals enlarge the defects in the cathode and ultimately appear on the cathode surface as large grains. These crystallized features can be seen in Fig 1.17. This irregular surface could potentially affect the interfacial adhesion between the organic and metal layers, further weakening device stability. Delamination occurs because the crystalline $\mathrm{Alq}_{3}$ clusters, being thicker than the surrounding amorphous regions, lift the cathode, leading to loss of contact between $\mathrm{Alq}_{3}$ and the cathode. The areas with loss of contact become sites for non-emissive dark spots[39]. 


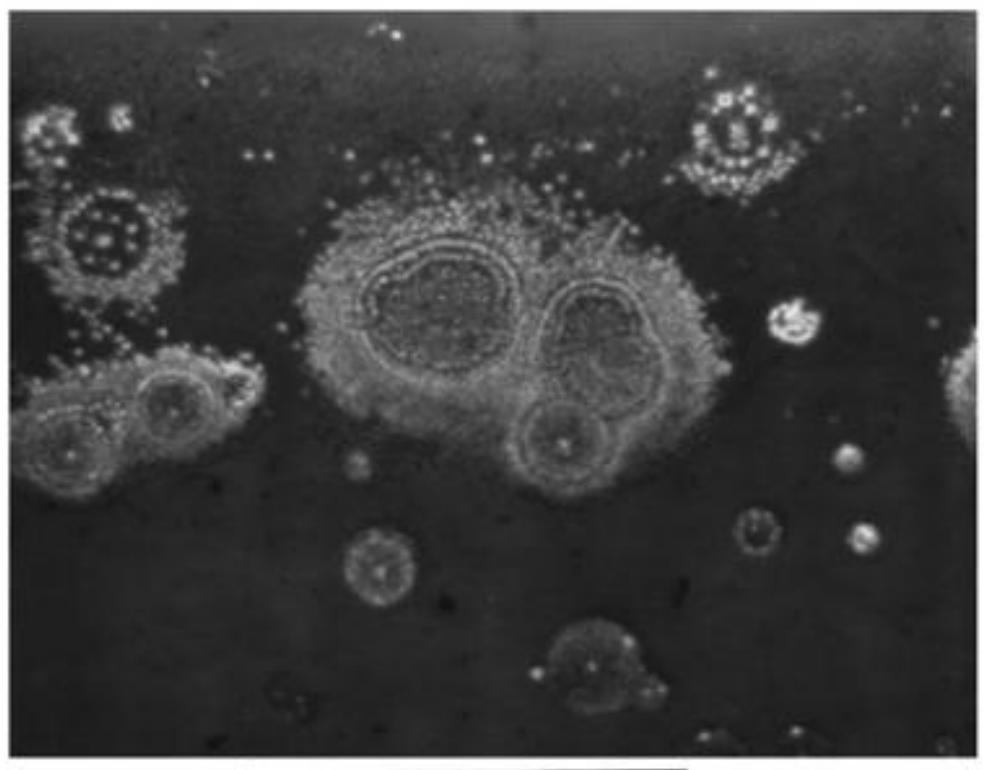

\section{$0.1 \mathrm{~mm}$}

Figure 1.17: Transmission polarization micrograph of an $60 \mathrm{~nm} \mathrm{Alq}_{3}$ film stored in humid air at $100 \% \mathrm{RH}$ for $6 \mathrm{~h}$ showing $\mathrm{Alq}_{3}$ crystallization[39].

\subsubsection{Heating and Organic Material Crystallization}

As seen above, crystallization can be induced in $\mathrm{Alq}_{3}$ as a result of moisture exposure. However Joule heating, as a result of current flow, can also induce crystallization in organic materials. In addition, direct heating(not due to Joule heat) of the organic layers like the hole transport layer(HTL) and/or the electron transport layer(ETL) above their respective glass transition temperatures $\left(\mathrm{T}_{\mathrm{g}}\right)$ can also result in crystallization of the amorphous organic matter .

For further discussion, we will consider the effects of heating on NPB and $\mathrm{Alq}_{3^{-}}$as these are the materials used in this thesis for hole transport and electron transport respectively. The choice of these materials will be discussed in section 1.9.

Glass transition of NPB is approximately $95-96^{\circ} \mathrm{C}$ [40]. Studies have shown that annealing(post-deposition heating) NPB at temperatures greater than $120-135^{\circ} \mathrm{C}$ [50] results in crystallization of NPB which aids in decreasing hole mobility in the OLED. This balances the hole-electron mobility in the OLED leading to improved device performance. In this aspect crystallization can prove to be advantageous. However, NPB crystallization has no direct impact 
on the electron injection at the cathode-organic interface, nor any direct relationship with the cathode-organic interfacial processes, and will not be discussed in this thesis.

Literature shows that crystallites are comparatively less accessible to moisture and hence less susceptible to chemical decomposition[42-43]. However, one must consider that high degree of crystallization also induces an irregular surface morphology. Crystallization also leads to the formation of gaps or pinholes between grains created upon phase transition from amorphous to ploy-crystalline phase. Hence controlling the effect of heat on the ETL - $\mathrm{Alq}_{3}$ is crucial for cathode-organic interface integrity which in turn controls device lifetime and performance. For good cathode-organic integrity, $\mathrm{Alq}_{3}$ films should not exhibit small grain morphology as seen in amorphous organic materials, however, neither should they exhibit highly irregular crystalline morphology. Another important factor to consider with $\mathrm{Alq}_{3}$ is that the $\mathrm{T}_{\mathrm{g}}$ of $\mathrm{Alq}_{3}$ is near $130^{\circ} \mathrm{C}$ to $140^{\circ} \mathrm{C}$. This is not an ideal fabrication temperature for integration with flexible substrates. Hence, we will discuss the effect of heating below the crystallization temperature range of $\mathrm{Alq}_{3}$ and in range of flexible substrate tolerance temperatures $\left(\leq 100^{\circ} \mathrm{C}\right)$.

When $\mathrm{Alq}_{3}$ is deposited on top of the hole transport layer at room temperature, it is in amorphous state and exhibits rough surface morphology. Amorphous state exhibits small grain morphology with a high grain boundary density leading to a high number of percolation paths for moisture and oxygen. Studies have shown that by annealing(post-deposition heating) $\mathrm{Alq}_{3}$ deposited on top of $\mathrm{NPB}$ at $100^{\circ} \mathrm{C}$ - which is well below the $\mathrm{T}_{\mathrm{g}}$ of $\mathrm{Alq}_{3}\left(129-139^{\circ} \mathrm{C}\right.$ [44]) - the surface morphology of $\mathrm{Alq}_{3}$ becomes smooth and featureless"[45]. A more organized morphology exhibits fewer grain boundaries, which means fewer percolation paths for moisture and oxygen. Furthermore, studies have shown that $\mathrm{Alq}_{3}$ deposited at $100^{\circ} \mathrm{C}$ on top of ITO/NPB leads to better lifetime than annealed ITO/NPB/ $\mathrm{Alq}_{3}$ stack [45]. AFM scans reveal that substrate heating while $\mathrm{Alq}_{3}$ deposition $\left(100^{\circ} \mathrm{C}\right)$ leads to a slightly rougher morphology when compared to the annealed $\mathrm{Alq}_{3} / \mathrm{NPB} / \mathrm{ITO}$ stack[45]. This morphological change can be seen in Figure 1.18.This minimal roughness might be beneficial in regards to increasing cathode adhesion by increasing surface-adhesion area. Moreover, larger scale surface roughness due to bigger grains might likely results in more difficult moisture penetration. Hence substrate heating while

\footnotetext{
* Content from reference [45] is reproduced in this section with the permission of Elsevier.
} 
depositing $\mathrm{Alq}_{3}$ or annealing $\mathrm{Alq}_{3}$ at a temperature below $\mathrm{T}_{\mathrm{g}}$ of $\mathrm{Alq}_{3}$ can result in large grain films with advantageous properties in regards to device lifetime.
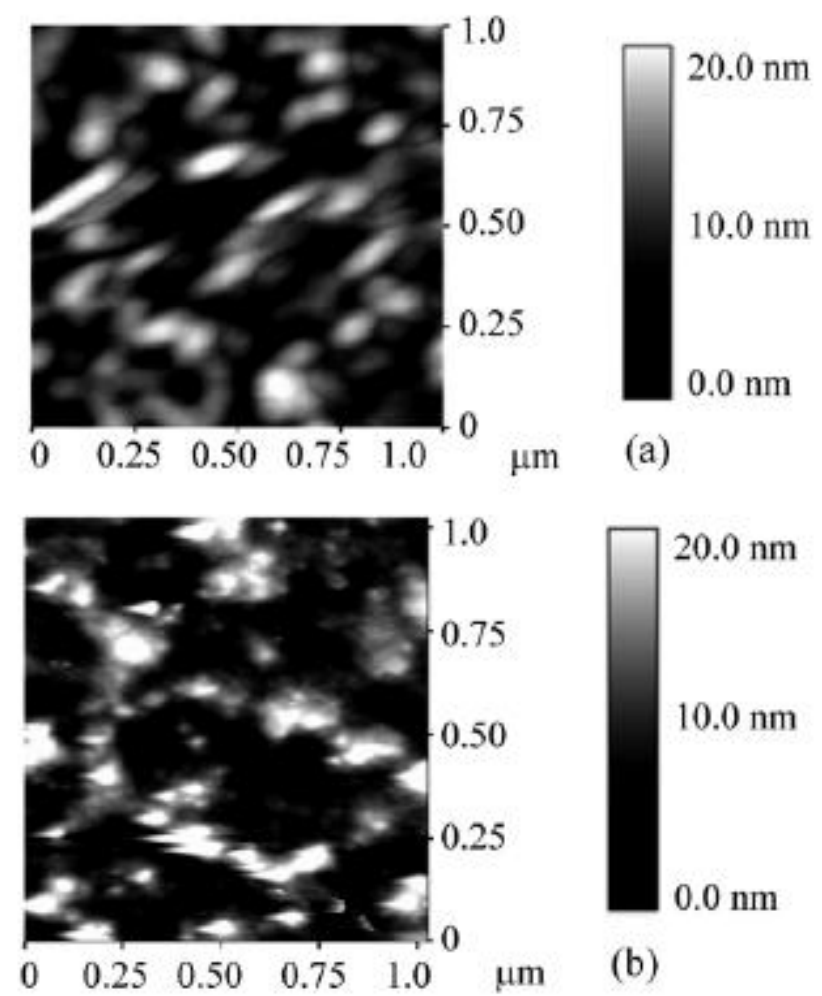

(b)
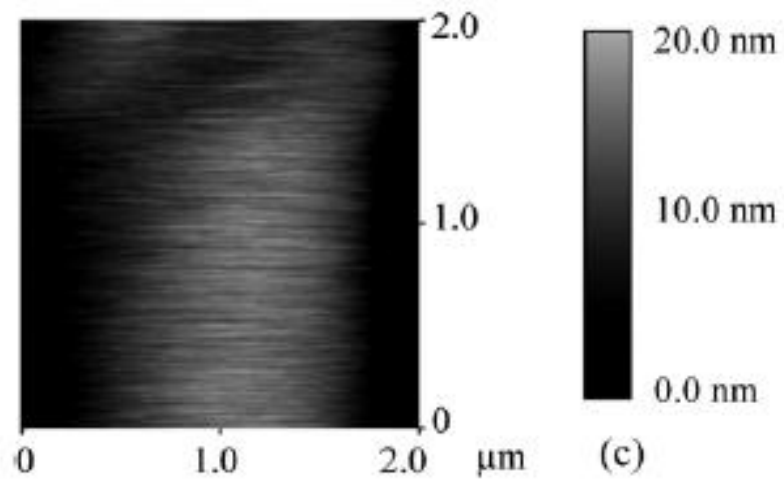

Figure 1.18: $\mathrm{AFM}$ scans of $\mathrm{Alq}_{3}$ layer (a) without heat treatment, (b)deposited at $65^{\circ} \mathrm{C}$, (c)annealed at $65^{\circ} \mathrm{C}$. The $\mathrm{Alq}_{3}$ layer here is deposited on top of a pre-existing room temperature deposited NPB layer[45].(Reprinted with permission of Elsevier) 


\subsection{Dark Spot Growth Processes}

In order to further understand dark spot degradation mechanism, the processes behind dark spot growth need to be identified. The propagation of dark spots is the primary cause behind the loss of emissive area in the OLED. Liew et al showed that the growth of dark spots occurs primarily due to cathode delamination[46]. Regular bi-layer OLEDs with NPB and $\mathrm{Alq}_{3}$ as HTL and ETL, respectively, were fabricated with a $\mathrm{Mg}: \mathrm{Ag} / \mathrm{Ag}$ cathode and then exposed to ambient conditions. After 24 hours storage in ambient conditions, dark spots were visible. The cathode was then peeled off with scotch tape and a new cathode of $\mathrm{Mg}: \mathrm{Ag} / \mathrm{Ag}$ was deposited on the device. As a result, the sites of previous dark spots due to ambient exposure were now emissive, except for a tiny non-emissive central spot[46]. This seems to provide direct proof that growth of dark spots is due to cathode delamination- since by depositing a new cathode (which is not yet delaminated) $\mathrm{Alq}_{3}$ electroluminescence could be obtained. Furthermore, when the cathode was re-deposited, the OLED showed bright circular features where the old dark spots used to exist[46]. This observation suggests that the growth of dark spots is associated with some changes in the underlying layers beneath the cathode. In addition, new dark spots that were not present in the device with the original cathode are now observed, suggesting that these new dark spots originate at the cathode or the $\mathrm{Alq}_{3}$ /cathode interface[46]. Fig 1.19 shows an optical micrograph of the OLED with the second cathode. After observing dark spot growth with the redeposited cathode, the second cathode was peeled off and another new $\mathrm{Mg}: \mathrm{Ag} / \mathrm{Ag}$ cathode was deposited. Similar trends of previous dark spot areas being emissive, as well as the occurrence of bright circular features in the area of the older dark spots were observed. This suggests that the cathode plays a role in the nucleation of dark spots and that the growth of dark spots and the associated bright circular features start at the $\mathrm{Alq}_{3} /$ cathode interface[46]. To make sure that these results were not only applicable to $\mathrm{Alq}_{3}$ and $\mathrm{Mg}: \mathrm{Ag} / \mathrm{Ag}$, different ETLs like Triphenyl Triazine (TPT) and different cathode like Ag were utilized, yielding the same results[46]. These experimental observations prove that cathode delamination is the primary cause for dark spot growth in OLEDs.

\footnotetext{
- Content from reference [46] is reproduced in this section with the permission of AIP.
} 


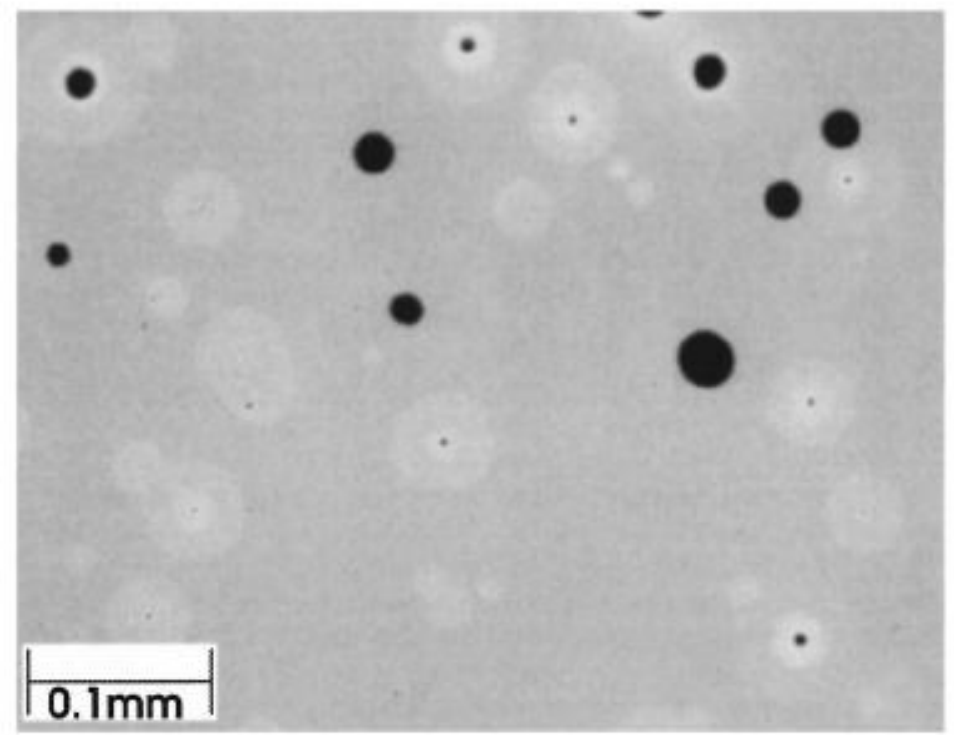

Figure 1.19: Dark spot and bright spots seen after cathode peel off and redeposition[46].(Reprinted with permission of AIP)

Now that the primary cause of dark spot growth has been determined to be cathode delamination, ways of controlling dark spot growth are discussed.

In the commercial market, the issue of dark spot growth is combated with the use of encapsulation to protect the OLEDs from ambient conditions. As mentioned before, encapsulation for flexible OLEDs is still considered challenging. In the next section, the issues with rigid and thin film encapsulation for flexible OLEDs are discussed.

\subsection{Encapsulation/Barrier Layers}

Traditionally, many organic electronic devices utilized glass/metal lids as barrier layers to protect against moisture/oxygen. An inert metal or glass layer was used as a "lid" which was sealed with an UV-cured epoxy resin. Getter materials were utilized in such structures. In some configurations, an inert gas has been mentioned to have been filled inside the voids to make the device extremely stable. Even though this approach does provide protection against 
moisture/oxygen permeability, it has flexibility issues as well as robustness issues. A device encapsulated with glass is prone to accidental breakage which can compromise the barrier structure leading to overall device deterioration. Glass encapsulation is also impossible to integrate into roll to roll OLED fabrication. A typical glass encapsulation structure can be seen in Fig 1.20.

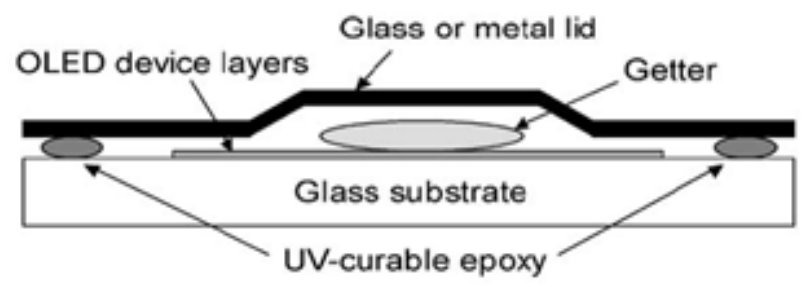

Figure 1.20: Traditional glass/metal rigid encapsulation[47].

In today's market, flexible OLEDs have great appeal due to the opportunities that they can enable. Rollable display screens, foldable maps, flexible lighting options are just few of the attractive market applications for OLEDs. Flexible OLEDs also have the potential to be thin and light weight. However, flexible OLEDs require flexible encapsulation structures. Furthermore, flexible OLEDs also require low temperature encapsulation procedures, so as not to damage the flexible substrate and the organic layers. The potential for flexible OLED fabrication via roll-toroll processing also stipulates another requirement for flexible OLED encapsulation - it should be applicable to large surface integration, making glass encapsulation obsolete.

Using flexible polymer lids might seem to be incrementally better than glass in the aspect of flexibility. However, polymer lids can lead to delamination while being folded. This can lead to device compromise. Hence, the only option for reliable encapsulation for flexible OLEDs is thin film encapsulation.

Thin film encapsulation layers provide easy integration into flexible OLEDs. If thin film encapsulation utilizes low temperature deposition processes, it can overcome all the obstacles that glass encapsulation encounters for flexible OLEDs. Moreover, thin film barrier layers are extremely thin and have the potential to be transparent unlike metal lids, and they are not as bulky as glass substrates and are easy to integrate into roll-to-roll production. In spite of their 
many advantages, thin film encapsulation layers have to maintain a high level of impermeability (to match glass encapsulation) to be considered viable due to the sensitive nature of OLEDs. Certain multi-layer thin film encapsulations utilizing different inorganic and/or organic layers have been shown to possess high impermeability to moisture/oxygen. Barix Encapsulation Technology by Vitex Systems is one of the most promising thin film encapsulation methodologies seen in today's industry. Barix multi-layer thin film encapsulation is discussed in the section below.

\subsubsection{Barix Encapsulation Technology-Thin Film Barrier Layers}

Even with good attributes such as flexibility and possible transparency, thin film barriers still need to meet the stringent requirements for water vapor and oxygen impermeability needed for organic electronics. A water vapor transmission rate (WVTR) of $10^{-6} \mathrm{~g} / \mathrm{m}^{2} /$ day and oxygen transmission rate (OTR) in the range of $10^{-3}-10^{-5} \mathrm{~cm}^{3} / \mathrm{m}^{2} /$ day is desirable for OLEDs. Figure 1.21 gives some WVTRs and OTRs requirements for various electronics to highlight the stringent needs of organic electronics.

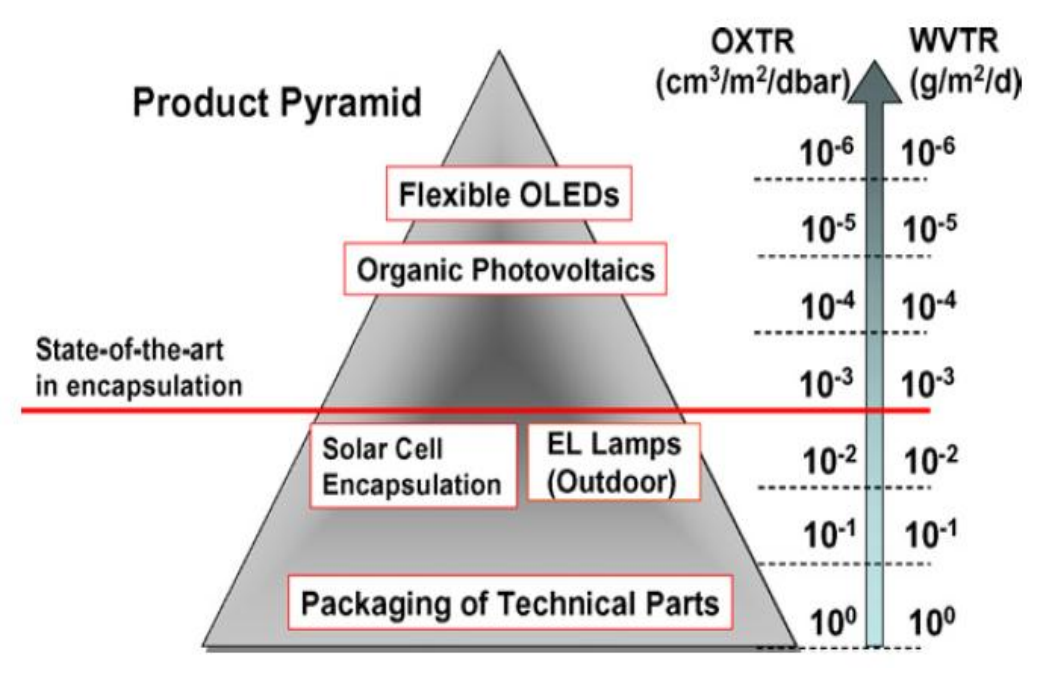

Figure 1.21: WVTR/OTR requirements for various electronics.

Barix Multilayer encapsulation represents a promising technique for protection from ambient conditions for flexible OLEDs. Oxygen permeation rates of $10^{-6} \mathrm{~cm}^{3} / \mathrm{m}^{2} / \mathrm{day}$ have been 
achieved via Barix encapsulation on a plastic substrate[48]. Barix multilayer encapsulation takes advantage of the redundancy and tortuosity provided by a multi-layer structure prolonging the permeation path of oxygen and/or moisture through the barrier layers. The multilayer stack consists of alternating organic and inorganic layers. The organic layers act as a planarization layer which helps in a smoother surface, whereas the inorganic layers(aluminum oxide) act as a permeation barrier to moisture and oxygen[48]. Barix encapsulation is also transparent and hence is suitable for top-emitter OLEDs too. Most importantly, the encapsulation deposition process is a low temperature one, making the process extremely suitable for flexible OLEDs.

Although the organic materials used in Barix are proprietary, the general deposition process is illustrated as seen in Fig 1.22. The organic materials are deposited using a nonconformal deposition technique - the organic material starts as a liquid which is then vaporized and cured(UV cure) to form a solid layer[48]. The inorganic aluminum oxide is deposited via DC sputtering. 4-5 polymer(organic)/inorganic dyads are used for encapsulation[48].

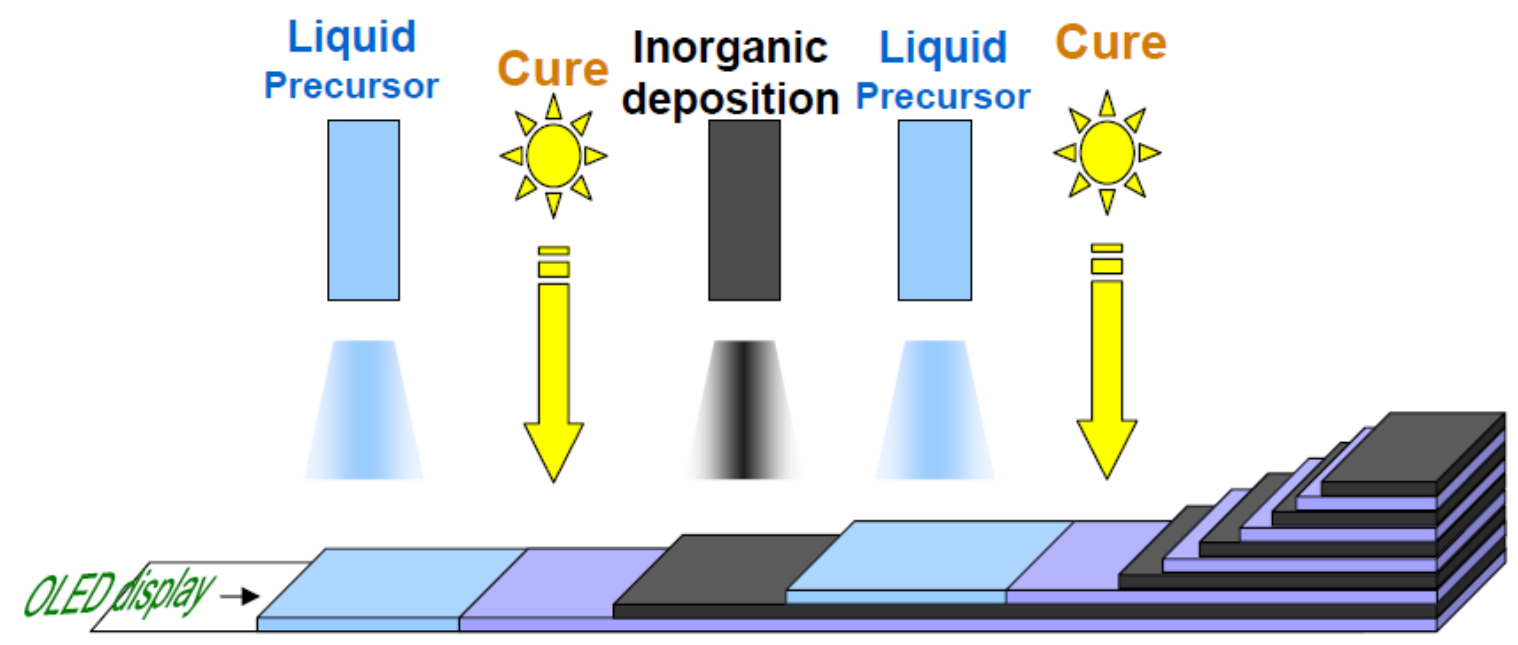

Figure 1.22: Barix Encapsulation Technique[48].

Drawbacks of multilayers structures like Barix are that they require complex post-OLED fabrication deposition with the need to have separate deposition techniques for different layers. Multilayer thin film encapsulation also results in long processing times which always introduces the potential for complications/contamination. Multiple constituent materials may also increase 
the cost-index for fabrication and thus make the fabrication of the encapsulated OLED potentially expensive.

Hence we can see that even the best thin film encapsulation introduces complexity and long processing times. Furthermore, Chwang et al. showed that Barix thin film encapsulation does not achieve the encapsulation integrity of glass barrier layers for flexible or rigid substrate OLEDs[49]. Therefore, a need exists to find alternative means of suppressing dark spot growth means that would not introduce complexity or potential for cost increase. We know that the cathode-organic interface is the main dark-spot formation site as a result of moisture $/ \mathrm{O}_{2}$ propagation through the pinholes in the metal cathode in the OLED. If the interfacial adhesion between the cathode and the organic layers was strong enough, the facile formation of metal hydroxide sites at the interface could potentially be hindered. By finding ways to strengthen the cathode-organic interface, and consequently the inherent resistance of the OLED structure to moisture and oxygen, suppression of dark spots can potentially be achieved.

\subsection{Alternative approaches to control dark spot growth}

As seen in section 1.7, encapsulation using rigid/glass structures has been shown to suppress dark spot growth. However, encapsulation of flexible OLEDs still presents a challenge due to the lack of high integrity, flex-integrable encapsulation . Even superior thin film encapsulation techniques like Barix by Vitex systems, produce OLEDs with higher dark spot growth when compared to glass encapsulated OLEDs[48]. Chwang et al[49], have demonstrated that the use of Barix encapsulation on plastic substrates results in quarter the half life of a glass encapsulated glass substrate OLED(half life: $2500 \mathrm{~h}$ vs. 10,000h respectively). For comparison, Barix encapsulation results in $3700 \mathrm{~h}$ half life for a glass substrate OLED vs. $2500 \mathrm{~h}$ for a plastic(flexible) substrate OLED[49]. Therefore, even superior commercial thin film barrier layers do not achieve the high integrity encapsulation which is seen with glass barriers. Due to this difficulty, alternative approaches for the suppression of dark spot growth need to be investigated. By investigating non-encapsulation approaches for dark spot suppression, the stringency of flexible encapsulation can be potentially eased. Literature shows the advantageous 
effects of annealing/heating OLEDs on the device lifetime and thereby their advantageous effects on controlling dark spot growth[40,42,43,50 ]. Furthermore the use of a graded cathode - a mixed organic metal layer(MOML)- by Aziz et al has also been shown to prolong device lifetime[53].

Hence to facilitate further dialogue on non-encapsulation related approaches for controlling dark spot growth, literature findings on the effect of substrate heating, postdeposition annealing, and the use of a cathode-organic interfacial layer on device performance and/or dark spot retardation are discussed below.

\subsubsection{Effect of Elevated Temperature Processing}

\subsubsection{Substrate Heating While Deposition}

Annealing of OLEDs has been shown to improve device lifetimes under carefully selected temperature ranges. In 1999, Gao et al ${ }^{\star}$ [40] showed the positive effect of elevated temperature processing on device lifetime of an OLED by heating individual layers of the OLED. Gao deposited the HTL (NPB) at $140^{\circ} \mathrm{C}$, the ETL $\left(\mathrm{Alq}_{3}\right)$ at $140^{\circ} \mathrm{C}$, and the cathode $(\mathrm{Al})$ at $60^{\circ} \mathrm{C}$. The resulting OLEDs showed $30 \%$ better luminance at $20 \mathrm{~mA} / \mathrm{cm}^{2}$ current density when compared to a non-heat treated OLEDs with the same organic and cathode layers[40]. This improvement in performance was mainly attributed to the HTL already being crystallized in the fabricated device, so further operation and/or storage would not affect the device negatively. Furthermore, when the devices were stored in $40 \%$ RH environment for 2 weeks, the heattreated devices continued to possess electroluminescence at $9 \mathrm{~V}$ [40]. On the other hand the nonheat treated devices exhibited no emission at all. Another set of OLEDs with only the HTL (NPB) deposited at $140^{\circ} \mathrm{C}$ were also fabricated. These devices were shown to possess electroluminescence at $9 \mathrm{~V}$ after 2 weeks exposure to $40 \% \mathrm{RH}$ conditions but they only possessed a slight increase in luminance over the non-heat treated OLEDs when compared to the luminance increase seen with the individually heated layer OLEDs[40]. In conclusion, the study by Gao et $\mathrm{al}[40]$, on the effect of heating the substrate while organic and metal deposition shows that NPB crystallization helps in improving device performance and heating $\mathrm{Alq}_{3}$ and metal might lead to a

\footnotetext{
^ Content from reference [40] is reproduced in this section with the permission of AIP.
} 
better adherent cathode interface(better electron injection). However, Gao et al[40], focused more on the crystallization of the NPB and its advantages on device performance rather than on the strengthening of adhesion between interfaces. No dark spot micrographs were presented.

In 2003, Chan et al ${ }^{\mathrm{r}}$ [41] showed that by depositing organic layers (NPB and $\mathrm{Alq}_{3}$ ) at higher temperatures like $160^{\circ} \mathrm{C}$, retarded dark spot growth was observed. After 1200min storage in air, almost $80 \%$ of the emissive area of a non-heat treated device became non-emissive. On the other hand, dark spots on the $160^{\circ} \mathrm{C}$ deposited organic layer OLEDs were still small in diameter (most $<0.07 \mathrm{~mm}$ ) as shown in Fig 1.23[41]. No direct reasoning for the dark spot retardation in relation to the heating of the ETL $\left(\mathrm{Alq}_{3}\right)$ was given. The focus was primarily on heat-induced crystallization of NPB as seen in the work by Gao et al[40] in 1999.

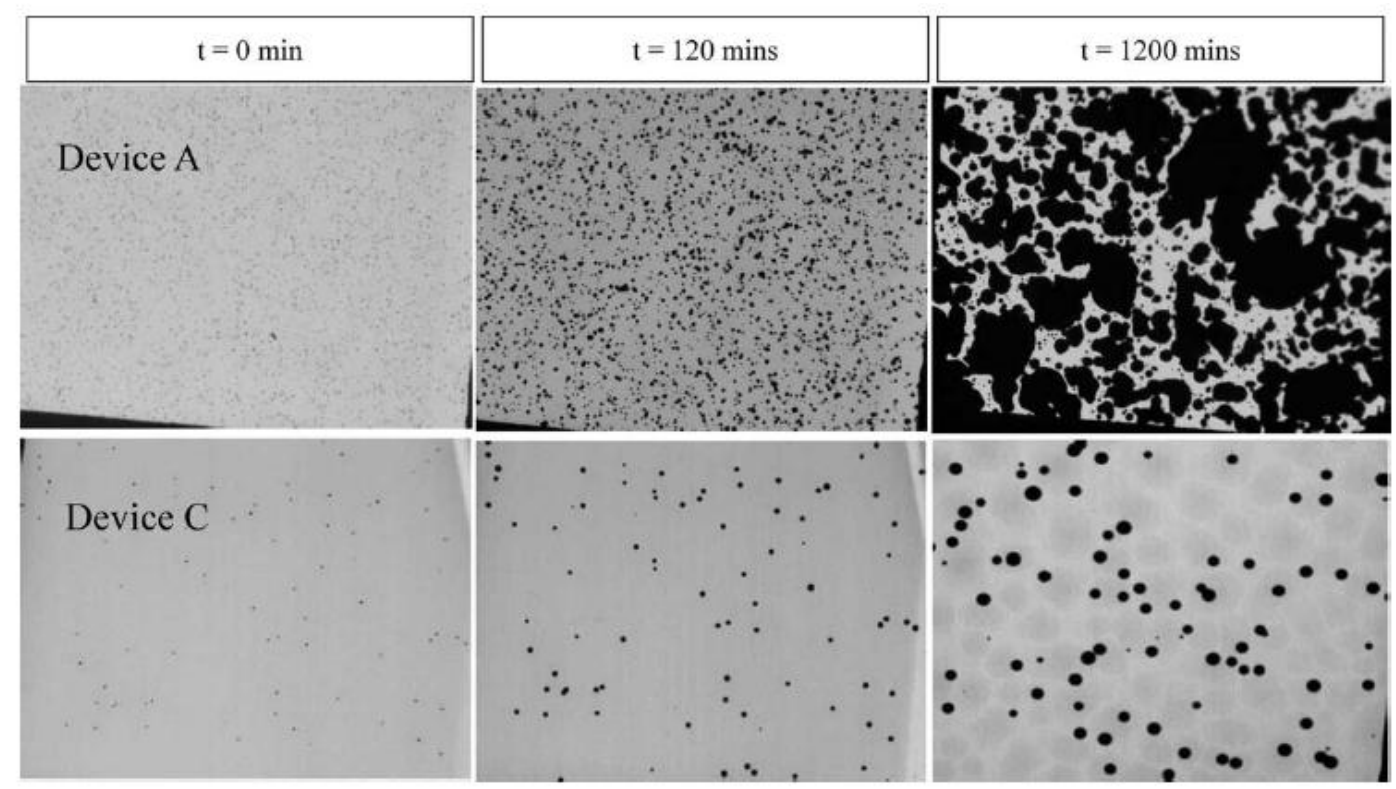

Figure 1.23: Dark spot growth at $0,120,1200$ mins exposure to $40 \% \mathrm{RH}$.Device $\mathrm{A}$ is not heated treated, Device $\mathrm{C}$ has its HTL and ETL deposited at $160^{\circ} \mathrm{C}[41]$. (Reprinted with permission of Elsevier)

\footnotetext{
${ }^{\Upsilon}$ Content from reference [41] is reproduced in this section with the permission of Elsevier.
} 


\subsubsection{Post-deposition annealing}

Another way to utilize the effect of heat on OLEDs is to anneal the OLED postfabrication for a specific amount of time. In 2005, Sun et $\mathrm{al}^{\Lambda}$ [50]showed that by annealing an OLED (structure: ITO/NPB/ $\mathrm{Alq}_{3} / \mathrm{Al}$ ) at $120^{\circ} \mathrm{C}$ for one hour lead to marked improvement in luminescent efficiency, brightness, and operating stability when compared to a non-annealed device. The maximum luminance of a $120^{\circ} \mathrm{C}$ annealed device was $6240 \mathrm{Cd} / \mathrm{m} 2$ as compared to a mere $3650 \mathrm{~cd} / \mathrm{m}^{2}$ for a un-annealed device[50]. Furthermore OLEDs were selectively annealed layer by layer to focus on the effect of heating on progressively deposited layers. Table 1.1 shows that by annealing(i.e. post deposition heating) the device -which includes the Al cathodeat $120^{\circ} \mathrm{C}$, the best luminance $\left(670 \mathrm{~cd} / \mathrm{m}^{2}\right)$ and efficiency $(3.5 \mathrm{~cd} / \mathrm{A})$ values were obtained[50].

Table 1.1 OLED characteristics for various annealing strategies[50]. (Reprinted with permission of Elsevier)

\begin{tabular}{|c|c|c|c|c|c|}
\hline & Layer(s) annealed & $\begin{array}{l}\text { Voltage (V) at } \\
100 \mathrm{~cd} / \mathrm{m}^{2}\end{array}$ & $\begin{array}{l}\text { Luminance }\left(\mathrm{cd} / \mathrm{m}^{2}\right) \text { at } \\
20 \mathrm{~mA} / \mathrm{cm}^{2}\end{array}$ & $\begin{array}{l}\text { Maximum efficiency } \\
(\mathrm{cd} / \mathrm{A})\end{array}$ & $\begin{array}{l}\text { Maximum luminance } \\
\left(\mathrm{cd} / \mathrm{m}^{2}\right)\end{array}$ \\
\hline A & None & 8.8 & 280 & 1.9 & 3650 \\
\hline B & NPB & 9.8 & 570 & 3.0 & 4730 \\
\hline $\mathrm{C}$ & $\mathrm{NPB} / \mathrm{Alq}_{3}$ & 9.7 & 560 & 2.8 & 4790 \\
\hline D & $\mathrm{NPB} / \mathrm{Alq}_{3} / \mathrm{Al}$ & 9.5 & 670 & 3.5 & 6240 \\
\hline
\end{tabular}

The chosen temperature was $120^{\circ} \mathrm{C}$.

Furthermore, new OLEDs with the structure ITO/NPB/ $\mathrm{Alq}_{3} / \mathrm{LiF} / \mathrm{Al}$ were fabricated with individual layers selectively annealed just like done before[50]. Once again, luminance and luminance efficiency improvements were observed for the OLEDs with all the layers annealed(i.e. post deposition heating). Moreover, half-life improvement was also observed (52 hours for the device with all the layers annealed as opposed to just 14hours for the un-annealed device) as seen in Fig 1.24. These results shows that annealing the cathode leads to enhanced electron injection. AFM scans of the top of the Al cathode for the device with all the layers annealed showed that annealing leads evening out of the undulation of the Al film and hence leads to smoother morphology[50]. This could be the reason behind better operational stability.

\footnotetext{
${ }^{\Lambda}$ Content from reference [50] is reproduced in this section with the permission of Elsevier.
} 
Although storage stability and dark spot growth as a result of ambient exposure were not discussed, one can see how the heating of the cathode could be beneficial to ambient stability of the OLED. It can be speculated that better electron injection could be a result of a better adherent interface between the cathode and the organic layer as a result of heat.

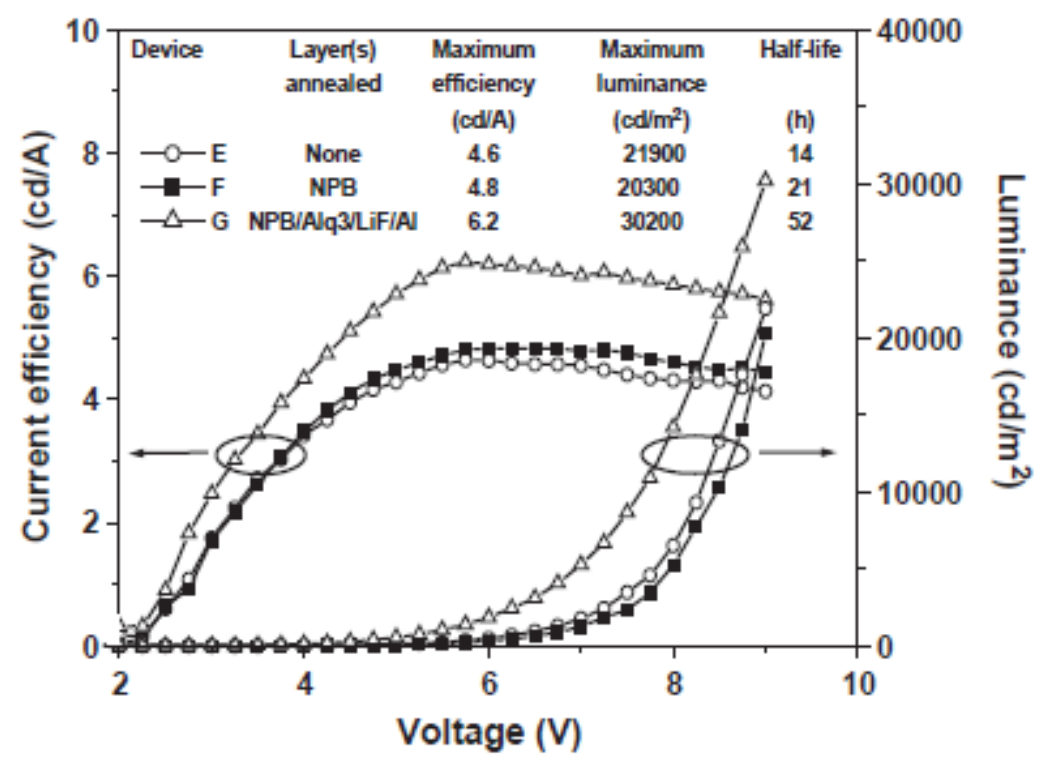

Figure 1.24: Current efficiency and Luminance characteristics for OLEDs with various layers being annealed . The general OLED structure is: ITO/NPB/ $\mathrm{Alq}_{3} / \mathrm{LiF} / \mathrm{Al}[50]$. (Reprinted with permission of Elsevier)

Investigations into the effects of annealing(i.e. post-deposition heating) on OLED operation conducted by several other groups also show positive results[51-52]. Chen et al [51] showed that by annealing OLEDs (ITO/NPB/ $\mathrm{Alq}_{3} / \mathrm{Mg}: \mathrm{Ag} / \mathrm{Ag}$ ) at $100^{\circ} \mathrm{C}$ which is much below the $T_{g}$ of NPB, high current density and lower driving voltages could be achieved. The power efficiency could be improved by more than $40 \%$ by annealing at $100^{\circ} \mathrm{C}$ [51]. The reasoning behind the improved performance was attributed to improved interface structures between the HTL and ETL organic materials[51]. However, since the entire OLED was annealed at $100^{\circ} \mathrm{C}$, one may yet again presume some improvement in interface structure of the cathode and $\mathrm{Alq}_{3}$. Unfortunately this study did not focus on dark spot retardation as a result of annealing(i.e. post deposition heating). 
This uninvestigated issue was explored by Wong et $\mathrm{al}^{\vartheta}$ in 2006[52]. OLEDs (ITO/CFx ${ }^{+} / \mathrm{NPB} / \mathrm{Alq}_{3} / \mathrm{Mg}: \mathrm{Ag}$ ) were subject to mild annealing (i.e. post deposition heating) at $70^{\circ} \mathrm{C}$ (well below the $\mathrm{T}_{\mathrm{g}}$ of NPB) for $7.5 \mathrm{mins}$ and $15 \mathrm{mins}$. Dark spot measurements were recorded at 0mins,120mins, and 1200mins to ambient exposure. As seen in Fig 1.25, after 1200 mins of ambient exposure, almost $40 \%$ of the emissive area of the untreated device became non-emissive[52]. On the other hand, the dark-spot in the 7.5 and 15-min heated devices were still small in diameter (most less than $0.08 \mathrm{~mm}$ )[52]. The 7.5-min heated device gave the slowest dark spot growth. It is considered that in the heat treated devices, the mild heating might have modified and enhance the bonding at the various interfaces in the devices. In particular, there would be intermixing of molecules at the $\mathrm{NPB} / \mathrm{Alq}_{3}$ interface. Yet again, in this study, the cathode-organic interface was not the focus of the rationale behind retarded dark spot growth.
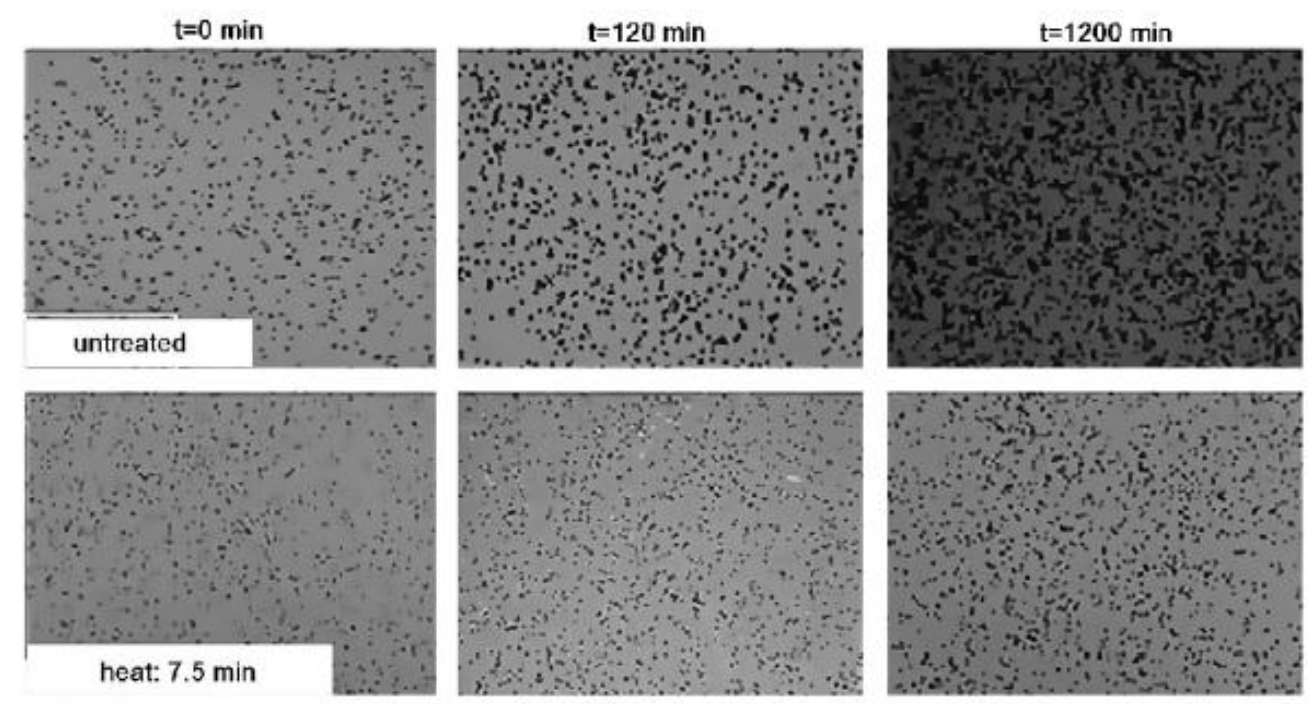

Figure 1.25: Dark spot growth at $0,120,1200$ mins exposure to $40 \% \mathrm{RH}$. The heated device is subject to a temperature of $70^{\circ} \mathrm{C}$ [52]. (Reprinted with permission of Elsevier)

A comparison of annealing vs. substrate heating while $\mathrm{Alq}_{3}$ deposition was conducted by Kwong et $\mathrm{al}^{*}[45]$. This study showed that substrate heating while deposition of $\mathrm{Alq}_{3}$ leads to superior OLED device performance[45]. It was found that the deposition at elevated substrate temperature improves both the maximum luminance and the stability of $\mathrm{Alq}_{3}$ based OLEDs.

\footnotetext{
${ }^{9}$ Content from reference [52] is reproduced in this section with the permission of Elsevier.

${ }^{+}$CFx: Fluorocarbon layer.

* Content from reference [45] is reproduced in this section with the permission of AIP.
} 
Annealing at $100^{\circ} \mathrm{C}$ resulted in inferior device performance. The differences in the performance of devices subjected to different temperature treatments were attributed to the changes in the film morphology, possible changes in the molecular packing, and different charge transport properties[45]. The morphology of $\mathrm{Alq}_{3}$ when it is deposited at $100^{\circ} \mathrm{C}$ is shown to be rougher than when $\mathrm{Alq}_{3}$ is annealed at $100^{\circ} \mathrm{C}$ as seen in Fig 1.18[45]. The rougher morphology of $\mathrm{Alq}_{3}$ might affect the cathode- $\mathrm{Alq}_{3}$ interface in a positive manner by improving current injection. Even though this study focuses on the morphology of $\mathrm{Alq}_{3}$ as a effect of annealing and substrate heating while deposition, it does not correlate this to dark spot retardation.

Hence after a survey of literature, we see widespread observations on the advantages of annealing and/or heating OLEDs but very little attention is focused on the direct improvement of cathode-organic interfacial characteristics - especially adhesion - as a result of annealing or heating the OLED/OLED layers.

\subsubsection{Effect of Cathode-organic interfacial Mixed Organic Metal Layer}

Aziz et al [53] have showed that the use of a Mixed Organic Metal Layer (MOML) deposited between the ETL and the cathode can result in dark spot retardation. Structures of OLEDs with a MOML interfacial layer(A) and without the MOML(B) are shown in Fig 1.26.

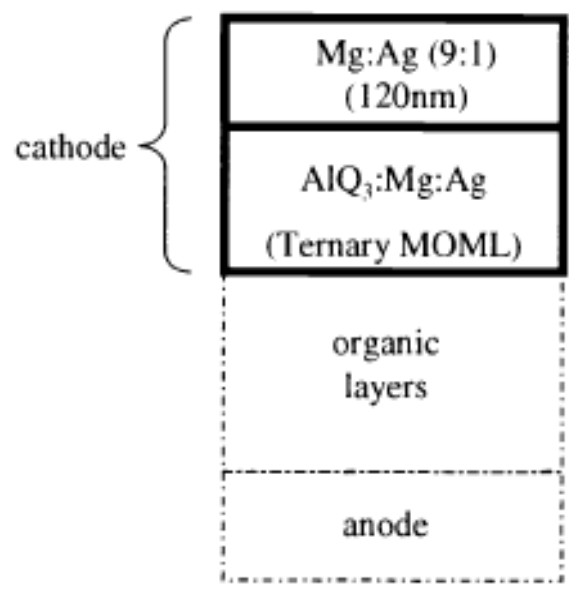

Device A

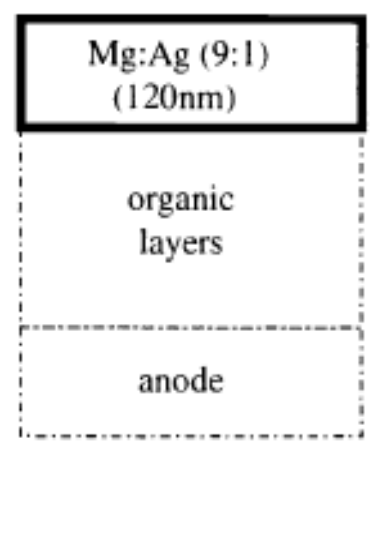

Device C

Figure 1.26: Device A shows the structure of an OLED with a MOML cathode-organic interfacial layer. Device B represents the general non-MOML OLED structure[53]. 
Fig. 1.27 shows micrographs obtained from device A and device $\mathrm{C}$ after 24 hours storage in the ambient $\left(22^{\circ} \mathrm{C}\right.$, RH: 55\%-60\%) without encapsulation[53]. Clearly, the use of MOML retards the growth of dark spots. It is interesting to point out that some of the studied MOML OLEDs were found to be still operable after being left, without encapsulation, in the ambient for a year; a feature that conventional devices with standard metal cathodes fail to show[53]. The causes behind the slower dark spot growth in MOML OLEDs were not discussed in this study. However, since delamination at the cathode/organic interface is believed to cause dark spot growth, the increased dark spot retardation in MOML OLEDs may be an indication of better interfacial adhesion at MOML-organic interface.

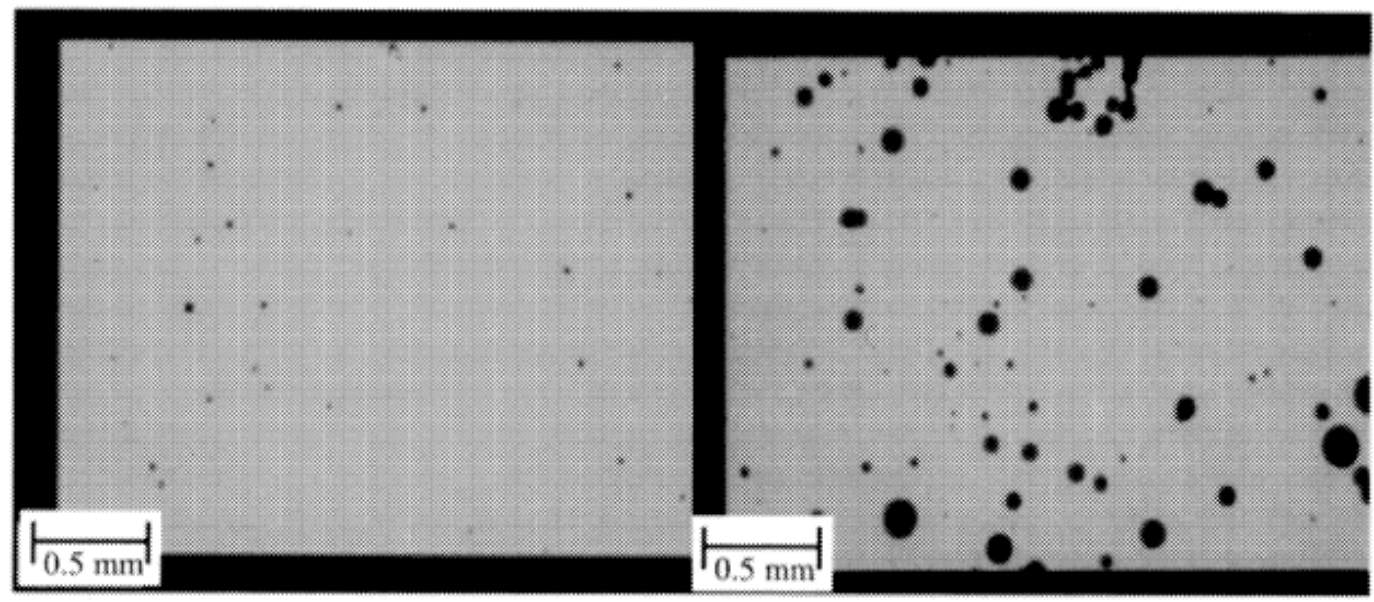

Figure 1.27: Dark spot growth after 24 hours ambient exposure for an OLED with a MOML layer (left) and an OLED without a MOML layer (right)[53].

Hence we have discussed the literature findings on the effects of heat and interfacial MOML layers on OLED performance improvement and/or dark spot retardation. Overall the effect of controlled and judicial heating on dark spot retardation is shown to be promising. However no substantiated studies exist on the direct effect of elevated temperature cathode deposition and/or the effect of using cathode-organic interfacial layers on improving dark spot retardation by focusing on the cathode-organic interfacial adhesion. Therefore, this work focuses on the role of cathode-organic interfacial adhesion in dark spot retardation. 


\subsection{This Work}

\subsubsection{Objectives}

This work will focus on the following objectives:

1) Studying adhesion between the cathode and organic layers - especially the effect of elevated temperature cathode deposition ${ }^{\oplus}$ and the effect of using a graded MOML interfacial layer on cathode-organic interfacial integrity.

2)Improving the ambient stability of the OLED by modifying the cathode-organic interface with the use of cathode deposition at elevated temperature and interfacial layers.

\subsubsection{Approach}

This work utilizes small-molecule OLEDs for all experiments. As SM-OLEDs employ thermal evaporation procedures which yield well controlled and homogeneous films, high efficiency OLEDs can be fabricated. As thermal deposition equipment is accessible for this thesis research work, the choice of using SM-OLEDs is very appropriate N,N'-di(naphthalene-1yl)-N,N'-diphenyl-benzidine (NPB), Tris(8-hydroxyquinoline) aluminum $\left(\mathrm{AlQ}_{3}\right), \mathrm{Mg}: \mathrm{Ag} / \mathrm{Ag}$ are used as the HTL, ETL, and cathode materials, respectively. These constituent materials are chosen as they are established in literature as materials capable of producing efficient OLEDs and have been extensively used in OLED research. The investigations in this work consist of two major thrusts, listed below.

\footnotetext{
${ }^{\oplus}$ The organic layers are not subject to deposition substrate heating as crystallization of the organic layers is not the focus of this thesis. The focus is on studying changes at the cathode-organic interface. By isolating the effect of substrate heating while cathode deposition on the cathodeorganic interface, the interfacial changes can be carefully analyzed. Hence, the ETL and the HTL are not subjected to deposition-substrate heating.
} 


\subsubsection{Use of Elevated temperature cathode deposition}

The effect of elevated temperature cathode deposition on OLED dark spot suppression is investigated. The findings of the aforementioned technique are compared to results obtained from post-fabrication annealed OLEDs. Any dark spot suppression improvements as a result of elevated temperature cathode deposition are analyzed for possible causes including cathodeorganic interfacial adhesion strengthening. The methodology is seen in section 1.9.3.

\subsubsection{Use of cathode-organic interfacial layers}

The effect of using various cathode-organic interfacial layers on OLED dark spot suppression are investigated. Any dark spot suppression improvements as a result of using cathode-organic interfacial layers are analyzed for possible causes including cathode-organic interfacial adhesion strengthening. The methodology is seen in section 1.9.3.

\subsubsection{Methodology}

i. Fabrication: OLEDs are fabricated with different temperature treatments: elevated temperature cathode deposition, post-fabrication annealing, and with different cathodeorganic interfacial layers. A control set of OLEDs is also fabricated without the use of any elevated temperature processing or cathode-organic interfacial layers.

ii. Moisture exposure: The fabricated OLEDs are stored inside a controlled environment chamber with RH maintained at $100 \%$ in air.

iii. Dark spot growth Micrographs: Dark spot growth is monitored by recording micrographs of the emissive side of the OLEDs at different moisture exposure times. This is done to observe improvements in dark spot suppression as a result of heating.

iv. Voltage Measurements: Changes in voltage needed to drive a constant current density when compared to the initial voltage needed to drive a similar current density are recorded for different moisture exposure periods. Larger Voltage changes $(\Delta V)$ are related to devices with faster dark spot growth which have larger non-emissive area and hence, larger resistivity. 
v. Atomic Force Microscopy (AFM) measurements: AFM morphology scans of the top of the $\mathrm{Mg}: \mathrm{Ag} / \mathrm{Ag}$ cathode and the top of the organic layers (the cathode is peeled off with scotch tape) are conducted for OLEDs with elevated temperature cathode deposition and for the control OLEDs. This is conducted to verify if morphological changes at the cathode-organic interface are the primary reason behind dark spot suppression as a result of a elevated temperature deposited cathode.

vi. Organic-Cathode Interfacial Adhesion Testing: The interfacial adhesion between the organic electron transport layer(ETL) and cathode is tested for the various OLEDs. A four point bending tester is used to obtain the energy release rate between the $\mathrm{Alq}_{3}$ and the cathode layers. Further details of experimental setup can be found in section 2.2. Adhesion testing can provide proof regarding the strengthening of cathode-organic interfacial adhesion.

\subsection{Thesis Organization}

This thesis consists of six chapters.

Chapter one consists of an introduction to OLEDs, historical background of organic electronics, structure and operational physics of OLEDs, followed by degradation mechanisms found in OLEDs with a focus on dark-spot degradation, concluding with research objectives and thesis organization.

Chapter two is related to the experimental aspect of OLEDs including substrate preparation, device fabrication and device testing.

Chapter three discusses the effect of cathode deposition temperature on dark spot growth and cathode-organic interfacial adhesion. Various ways of performance analysis are chosen including dark spot growth micrographs, electrical measurements, AFM surface morphology measurements, as well as interfacial adhesion force measurements. 
Chapter four discusses the effect of using cathode-organic interfacial layers on dark spot growth and cathode-organic interfacial adhesion. Adopting some of the performance analysis techniques seen in chapter three, the advantages of using a cathode-organic interfacial layer on dark spot suppression are analyzed.

Chapter five describes novel thin-film encapsulation layers using Titanium and Molybdenum Oxide. The viability of such an encapsulation layer is briefly analyzed.

Chapter six provides conclusions of the experimental works seen in chapters three and four, and recommendations for future work. 


\section{Chapter 2 Experimental Methods}

Experimental methods involve the fabrication and testing of OLEDs. In this chapter, the fabrication of OLEDs is first introduced starting with substrate cleaning and ending with material deposition. After that, measurement equipment and techniques used to investigation of the characteristics of OLEDs are introduced.

\subsection{Fabrication of OLEDs}

Device fabrication includes substrate cleaning and thermal evaporation of the organic and metal materials. Each of these steps substantially determines the quality and performance of the operating OLEDs.

\subsubsection{ITO Substrate Cleaning}

The glass substrates used for fabricating OLEDs have pre-patterned $\operatorname{ITO}(15 \Omega / \mathrm{sq})$ on it as seen in Fig 2.1.

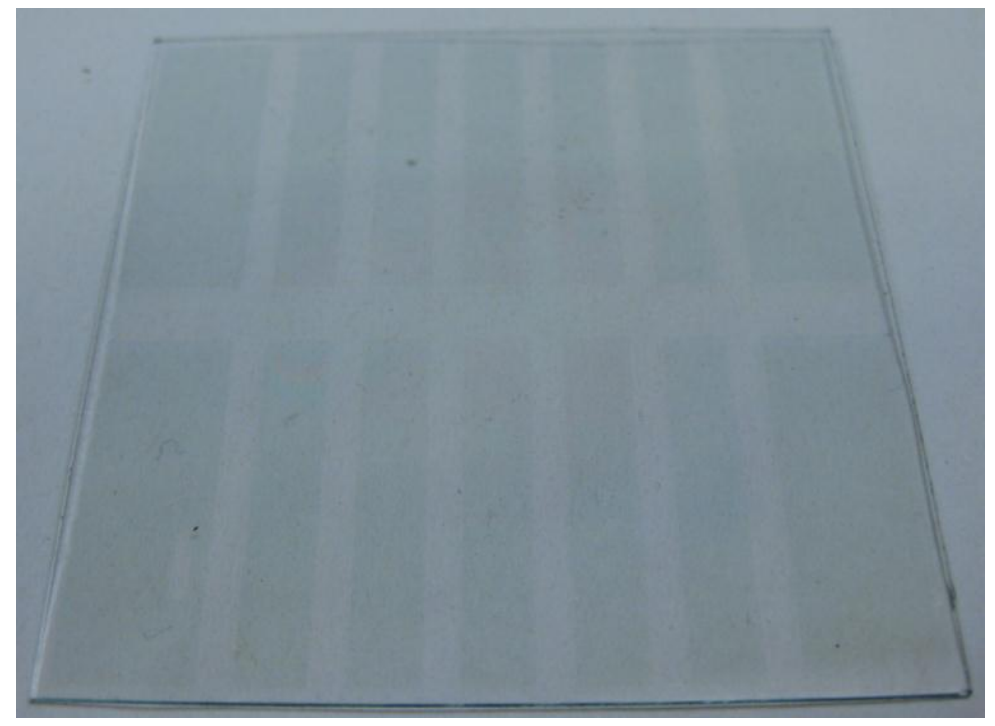

Figure 2.1: Patterned ITO substrate. 
The ITO substrate is submerged into a $1 \mathrm{~L}$ beaker with $500 \mathrm{~mL}$ of acetone and washed in an ultrasonic wash for 15 mins. Then the ITO substrates are taken away from solution and gently agitated with Q-tips for about 1 minute to further clean the ITO surface.

The substrate is then submerged in $500 \mathrm{~mL}$ of isopropanol and is subjected to another $15 \mathrm{~min}$ ultrasonic wash. A nitrogen gun is used to dry the substrate after the ultrasonic washing and blow away any extra solution on the substrate surface. Next the substrate is baked in an oven for $125^{\circ} \mathrm{C}$ for $30 \mathrm{mins}$ for further drying.

\subsubsection{Plasma Treatment}

Before the device fabrication, further cleaning of the ITO substrate by using reactive ion etching (RIE) technology is conducted. This is because RIE applies plasma treatment to the ITO surface in order to improve the work function of ITO, which greatly improves the charge balance and device lifetime. The optimal parameters of RIE for substrate treatment are listed in Table 2.1 .

Table 2.1 Optimal parameters of RIE for substrate treatment.

\begin{tabular}{|c|c|}
\hline Parameter & Value \\
\hline Pressure & 20 mTorr \\
\hline Inductively Coupled Plasma Power & 100 watts \\
\hline Duration & $2 \mathrm{~min}$ \\
\hline Gas proportion & $\mathrm{CF}_{4}(75 \%): \mathrm{O}_{2}(25 \%)$ \\
\hline Gas rates & $\mathrm{CF}_{4}(15 \mathrm{sccm}): \mathrm{O}_{2}(5 \mathrm{sccm})$ \\
\hline
\end{tabular}

\subsubsection{Deposition Parameters}

In all the devices, ITO and Mg:Ag are used as hole-injecting anode and electron-injecting cathode , respectively. N,N'-di(naphthalene-1-yl)-N, $\mathrm{N}^{\prime}$-diphenyl-benzidine (NPB) is used as a hole transport layer (HTL). Tris(8-hydroxyquinoline) aluminum $\left(\mathrm{AlQ}_{3}\right)$ is used as electron transport layers (ETL). Specific device structures are described in the corresponding chapters of this thesis. All devices are fabricated by deposition of the organic materials and the cathode 
metals using thermal evaporation at vacuum base pressure of about $5 \times 10^{-6}$ Torr on UV ozonecleaned ITO-coated glass substrates. In general, the deposition rates for HTL, ETL, and metal layers are listed in Table 2.2.

Table 2.2 Deposition rates.

\begin{tabular}{|c|c|}
\hline Material & Deposition Rate(s) $\AA$ /s \\
\hline $\mathrm{NPB}$ & 1 \\
\hline $\mathrm{Alq}$ & 1 \\
\hline $\mathrm{Mg}: \mathrm{Ag}$ & $1.8: 0.2$ \\
\hline $\mathrm{Ag}$ & 1 \\
\hline
\end{tabular}

\subsubsection{Cathode deposition at elevated temperature}

In certain device structures, the cathode is deposited at elevated temperatures inside the OLED fabrication chamber. A radiative heater inbuilt with the substrate holder is used to elevate the temperature to the desired value during cathode deposition. Heating ramp time (which includes temperature overshoot and temperature stabilization at set temperature) and overshoot above the target temperature for heating the substrate to $65^{\circ} \mathrm{C}$ are listed in Table 2.3. Overshoot above the target temperature should be minimum as excess heating above the target temperature is not desirable. Since the heater used in this work stabilizes at the set temperature within 60 seconds with minimal overshoot, excess heating above set temperature is avoided.

Table 2.3 Heating Parameters.

\begin{tabular}{|c|c|}
\hline Parameter & Value \\
\hline Average Ramp Time from $22^{\circ} \mathrm{C}$ to $65^{\circ} \mathrm{C}$ & 60 seconds \\
\hline Temperature Overshoot above $65^{\circ} \mathrm{C}$ & $5-7^{\circ} \mathrm{C}$ \\
\hline
\end{tabular}

\subsubsection{MOML layer deposition}

Certain OLED structures discussed in the subsequent chapters utilize a Mixed Organic Metal Layer (MOML) as an interfacial layer between the cathode and the ETL $\left(\mathrm{Alq}_{3}\right)$ layer. The layer composition and deposition rates of the MOML are listed in Table 2.4. 
Table 2.4 MOML composition information

\begin{tabular}{|c|c|}
\hline Parameter & Value \\
\hline Composition & $\mathrm{Alq}_{3}: \mathrm{Mg}: \mathrm{Ag} 42.5 \%: 42.5 \%: 5 \%$ \\
\hline Rate(s) & $\mathrm{Alq}_{3}: \mathrm{Mg}: \mathrm{Ag} 1.8: 1.8: 0.2 \AA / \mathrm{s}$ \\
\hline
\end{tabular}

This MOML composition is the same as seen in the works by Aziz et al in their development of the Black Cathode[53].

\subsection{Testing Methods}

i. Moisture-exposure degradation: The fabricated OLEDs are subjected to a100\% RH conditions in air. This is done by placing the OLEDs inside a controlled environment chamber maintained at $100 \% \mathrm{RH}$. The chamber is filled with ambient air, thereby subjecting the OLEDs to $100 \% \mathrm{RH}$ conditions in air.

ii. Voltage measurements: Voltage measurements ( $\Delta \mathrm{V}$ as mentioned in section1.9.3) are carried out in a nitrogen atmosphere with the use of a Fluke 114 True RMS Multimeter to monitor voltage differentials over time.

iii. Micrographs: Micrographs are taken for time-lapse (2,17/20,27hrs moisture soak) dark spot growth measurements- as seen on the light emitting side of the OLED with the help of Panasonic DMC-TZ3 and Minolta Chromameter CS-100. The OLEDs are photographed inside a nitrogen atmosphere so as to not induce additional ambient degradation during testing. Image analysis of the micrographs is conducted using GIMP 2.0 image authoring software. This software is used to analyze the dark spot coverage using its histogram tool.

iv. AFM Morphology Scans: AFM scans are carried out with the help of Dimension 3100 Scanning Probe Microscope. AFM morphology scans of the top side of the 
cathode and the organic layer are performed. The organic layer is accessed by peeling away the top cathode layer with the help of $3 \mathrm{M}$ scotch tape.

v. Bending tester- interfacial adhesion testing: Cathode-organic interfacial adhesion testing is carried out with the use of a home-built bending tester. The tester utilizes the four-point bending method to characterize adhesive fracture strengths at the cathode-organic interface. The tester consists of a linear actuator which provides vertical downward force for the four point bend setup. The four point bend setup requires a sample structure such that the layers on either side of the interface in question are sandwiched between two substrates with the help of fast sealing epoxy glue (Lepage Speed Set epoxy glue - 15minutes setting time). A pre-crack is cut into glass substrate 2 to a depth of half of the substrate's thickness. The sample is loaded into a four-point bend tester setup as illustrated in Fig 2.2.

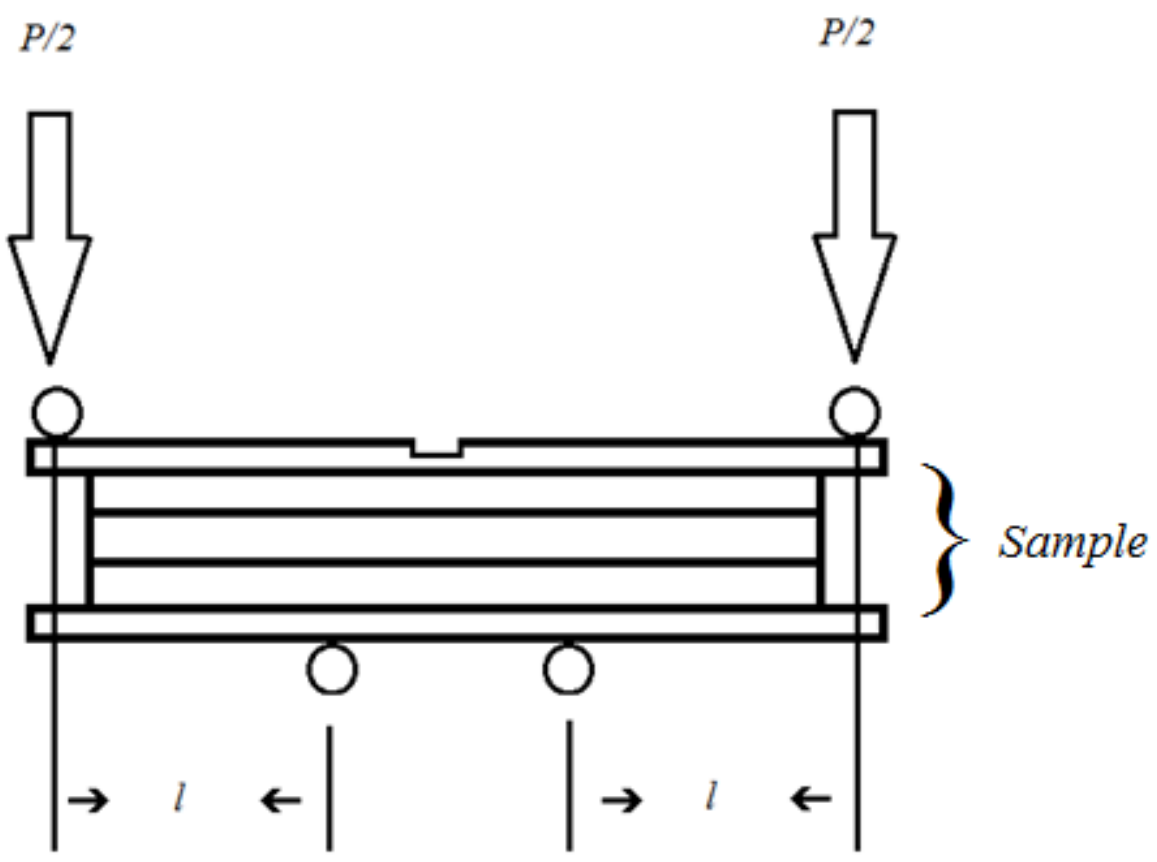

Figure 2.2: Four point Bend Configuration. $P$ is the total force exerted onto the sample, $l$ is the moment arm between the inner and outer pins. 
Once the sample is loaded onto the bending apparatus, the vertical distance between the top and bottom pins is gradually decreased by setting a displacement rate of $250 \mathrm{~nm}$ per second. As force is applied to the sample, a linear load region is seen as seen in Fig 2.3. As the load is increased, the pre-crack propagates down through the substrate until it propagates to the weakest interface (which is the interface of $\mathrm{Alq}_{3}$ and $\mathrm{Mg}: \mathrm{Ag}$ ). This event results in a sharp load drop also seen in Fig 2.3. Once the $\mathrm{Alq}_{3}-\mathrm{Mg}: \mathrm{Ag}$ interface has been compromised via the crack, the crack follows the interface in a horizontal direction. When the crack front propagates far enough from the initial pre-crack (distance $>$ two times wafer thickness)[54], stress intensity becomes constant. This is when a plateau is observed in the force vs. displacement curve. The value of load at this plateau can be used to calculate the energy release rate as seen in the equation below[55]:

$$
G=\frac{21 *\left(1-v^{2}\right) * P^{2} * l^{2}}{16 * E * b^{2} * h^{2}}
$$

where $v$ is the Poisson's ratio of the bulk substrate, $\mathrm{E}$ is the elastic modulus of the bulk substrate, $\mathrm{P}$ is the total force exerted onto the sample, $\mathrm{b}$ is the width of the sample, $\mathrm{h}$ is the wafer's thickness, and $\mathrm{l}$ is the moment arm that is created between the inner and the outer pins. 


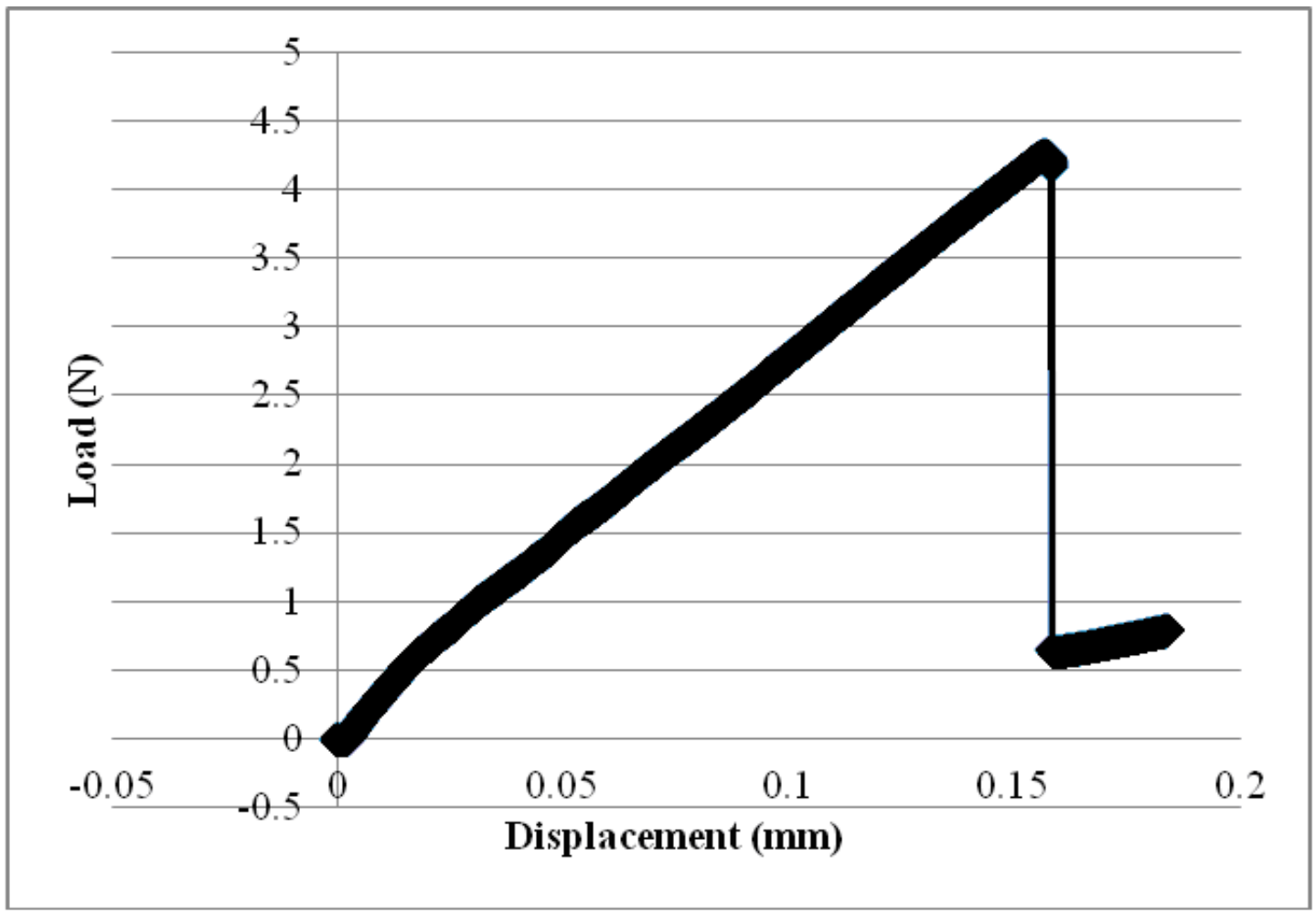

Figure 2.3: Typical Force vs. Displacement curve. This particular example uses a compromised $\mathrm{Alq}_{3} / \mathrm{Mg}$ :Ag interfacial stack showing a poor $(<1 \mathrm{~N})$ load at the interface.

The higher the energy release rate $(\mathrm{G})$, the stronger the interfacial adhesion is at the interface in question. This setup is utilized to record energy release rates at the cathode-organic interface for $65^{\circ} \mathrm{C}$ deposited cathode samples, $65^{\circ} \mathrm{C}$ annealed(i.e. post-deposition heating) samples, room temperature (RT) samples, samples with MOML layers between the $\mathrm{Alq}_{3}$ and cathode layers, and also samples with $\mathrm{LiF}$ and $\mathrm{Cs}_{2} \mathrm{CO}_{3}$ (electron injection layers-EILs) between the $\mathrm{Alq}_{3}$ and cathode layers. 


\section{Chapter 3 Effect of cathode deposition temperature on dark spot growth and cathode-organic interfacial adhesion}

A number of studies have shown the benefit of substrate heating/annealing on OLED lifetimes and characteristics. In their studies, Gao et al showed that better efficiency and stability can be achieved by high temperature deposition of the organic layers and the cathode[40].The study by Gao et al, focused more on the crystallization of the NPB and its advantages on device performance rather than on the strengthening of adhesion between interfaces. Increase in storage lifetime was mentioned, but dark spot growth was neither analyzed nor presented. More importantly, the effect of heating the cathode on device lifetime was not individually analyzed.

Another study by Wong et al [52] showed the advantageous effect of annealing the entire device, i.e. after deposition, at $70^{\circ} \mathrm{C}$ for 7.5 mins. Dark spot growth retardation was observed by this mild heat treatment. Hence, the judicious use of heat has been shown to be beneficial for prolonging device lifetime and efficiency. However the direct effect of heat on the cathodeorganic interface has not been investigated and still remains unclear. As the cathode-organic interface is a critical interface for cathode delamination and dark spot growth, the issue of heat on cathode-organic interfacial adhesion is investigated in this thesis.

In previous studies on the effect of subjecting the organic layers to high temperatures, it has been reported that depositing HTL and ETL at higher temperature resulted in a fused HTLETL interface[56]. The interface was mentioned to have lost its abrupt heterojunction property as a result of heat. Following this rationale, one can speculate that deposition of a metal onto an organic layer may improve the adherent forces between the layers as a result of a fused interface. This speculation will be the primary assumption in the series of experiments to follow.

As the primary focus of this research is to analyze cathode-organic interface adhesion and their correlation with dark spot growth, we are not interested in crystallization of organic layers and/or strong interdiffusion at the HTL/ETL interface as a result of heating. To ensure this, the cathode deposition temperature should be chosen below the $\mathrm{T}_{\mathrm{g}}$ of both $\mathrm{NPB}\left(95-96^{\circ} \mathrm{C}\right)[40]$ and $\mathrm{Alq}_{3}\left(129-139^{\circ} \mathrm{C}\right)[44]$, so as to not induce any unwanted crystallization. In addition, metal 
deposition temperature above $100^{\circ} \mathrm{C}$ in a study by Gao [42] was seen to cause shorting of the device. Due to these aspects, and also to keep the deposition parameters suitable for flexible OLEDs which need lower fabrication temperatures, the metal deposition temperatures are chosen to be $65^{\circ} \mathrm{C}$ and $80^{\circ} \mathrm{C}$. Initial experimental observations showed that heating the substrate to $65^{\circ} \mathrm{C}$ and $80^{\circ} \mathrm{C}$ produced near identical dark spot retardation results. Due to this, the lower temperature, i.e. $65^{\circ} \mathrm{C}$ is chosen as the definitive experimental temperature for metal deposition.

\subsection{Effect of elevated temperature cathode deposition on dark spot growth}

A group of OLEDs fabricated entirely at room temperature(RT) are used as a control group(GRP_1) and have the following structure: ITO/NPB(70nm)/ $\mathrm{Alq}_{3}$

(70nm)/Mg:Ag(100nm)/Ag(100nm). A subsequent group of OLEDs (GRP_2) are fabricated with a similar structure as GRP_1 with the difference being that for GRP_2 devices, cathode deposition is carried out at a substrate temperature of $65^{\circ} \mathrm{C}$. Fig 3.1 shows the general structure of GRP_1 and GRP_2 OLEDs. As explained in section 2.1.4, the substrate heater stabilizes at $65^{\circ} \mathrm{C}$ within 60 seconds after being switched on. The substrate heater is maintained at $65^{\circ} \mathrm{C}$ for a "soak" time of 15 minutes prior to cathode deposition. This is to ensure that the OLED temperature is uniform over the entire substrate. The cathode deposition takes a total deposition time of 25 minutes. Hence, the heater stays on for a total of 40 minutes. The heater is promptly switched off after metal deposition.

\begin{tabular}{|c|}
\hline Ag (100nm) \\
\hline Mg:Ag (100nm) \\
\hline Alq3 (70nm) \\
\hline NPB (70nm) \\
\hline ITO on glass \\
\hline
\end{tabular}

a)

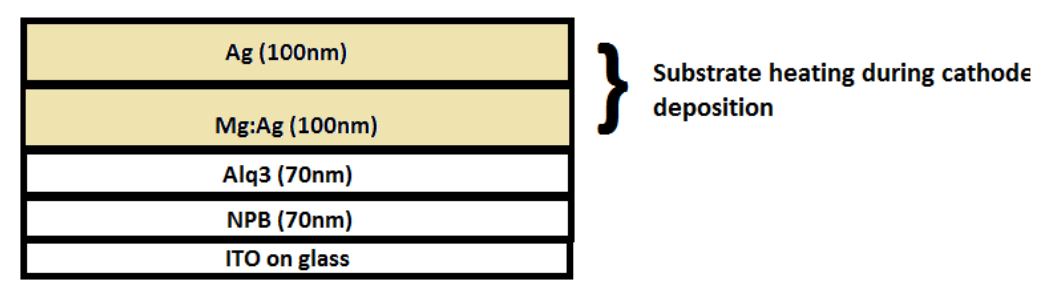

b)

Figure 3.1: OLED structure of GRP_1 (a) and GRP_2(b) devices 
Figure 3.2 shows micrographs of dark spot growth for GRP_1 versus GRP_2 OLEDs demonstrating growth of dark spots with respect to time. Micrographs are recorded at 2, 17, 27hrs after placing the OLED devices inside a controlled environment chamber, with relative humidity (RH) maintained at $100 \%$. It can be seen from Figure1 that the dark spot growth in GRP_2 is slower than the dark spot growth seen in group1 devices. 


\section{Room Temperature(RT) deposited OLEDs}
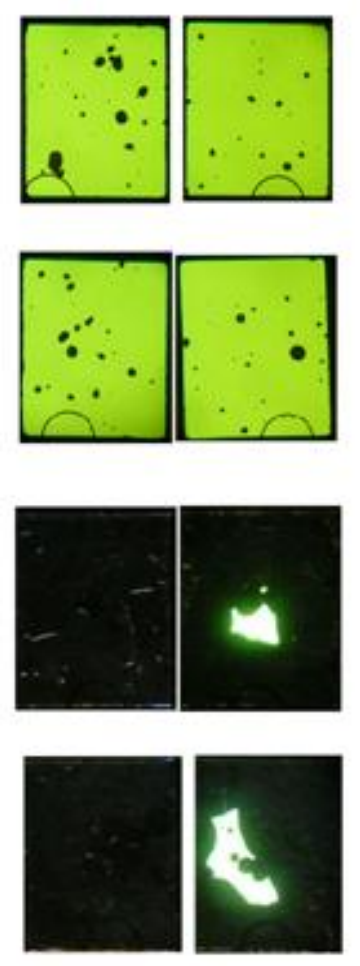

$65^{\circ} \mathrm{C}$ deposited cathode OLEDs
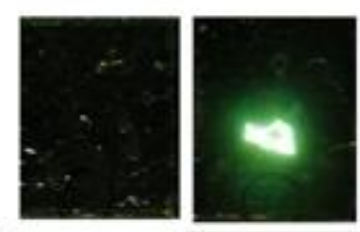

a)
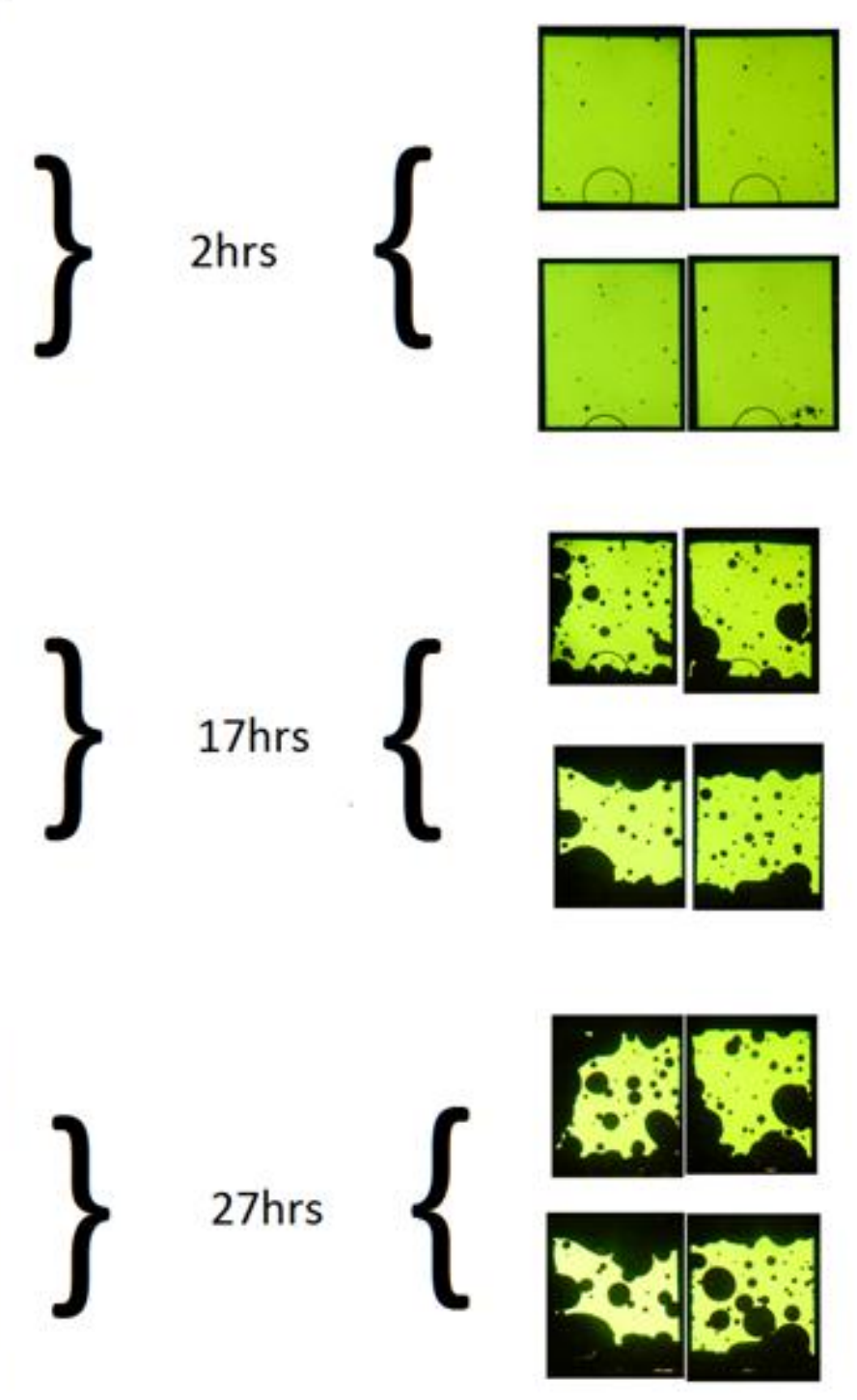

b)

Figure 3.2: Dark spot growth after 2,17,27hrs exposure to $100 \% \mathrm{RH}$ conditions for (a) GRP_1 OLEDs, and (b) GRP_2 OLEDs 
Image analysis of the micrographs using GIMP 2.0 shows that after 27 hours of exposure to $100 \%$ RH, GRP_2 OLEDs show dark spot coverage of only 43-54\% of total surface emissive area. On the other hand, the GRP_1 devices show severe device degradation with dark spot coverage of $95-100 \%$ of total surface emissive area. On average, $52.75 \%$ of the total OLED surface area still remains emissive when the cathode is deposited at $65^{\circ} \mathrm{C}$ as compared to only 2.0-2.5\% emissive surface area for room temperature deposited cathode OLEDs. This indicates that the deposition of cathode at $65^{\circ} \mathrm{C}$ helps in retarding the growth of dark spots. (Note: The black regions at the top and bottom of the devices are due to edge effects. Also, the black circular ring seen across all the images is the focus ring of the microscope, and can be ignored.)

When emissive surface area shrinks, the OLED becomes more resistive since the dark spots are essentially non-conductive regions. As a result, the voltage needed to drive a current density of $20 \mathrm{~mA} / \mathrm{cm}^{2}$ also increases with dark spots. Such increases in voltage should be expected to correlate with the extent of dark spot growth as seen in Fig 3.2. Henceforth, for all voltage increase discussions, we define $\Delta \mathrm{V}$ as the increase in voltage needed to drive a current density of $20 \mathrm{~mA} / \mathrm{cm}^{2}$ when compared to the initial voltage needed to drive a similar current density, recorded immediately after fabrication of the OLED. Fig 3.3 shows $\Delta \mathrm{V}$ values for GRP_1 and GRP_2 OLEDs after 0,2,17,27hrs exposure to 100\% RH. It can be seen from Fig 3.3 that $\Delta \mathrm{V}$ values for GRP_1 are larger when compared to GRP_2 devices. After 27hours of $100 \% \mathrm{RH}$ exposure, GRP_1 devices show an average $\Delta \mathrm{V}$ of nearly $7.568 \mathrm{~V}$ as opposed to $1.288 \mathrm{~V}$ $\Delta \mathrm{V}$ for GRP_2 devices. These results agree with the time-lapse micrographs of the OLED emissive surface. Hence it can be concluded that depositing the cathode at $65^{\circ} \mathrm{C}$ substrate temperature leads to definite dark spot suppression. 


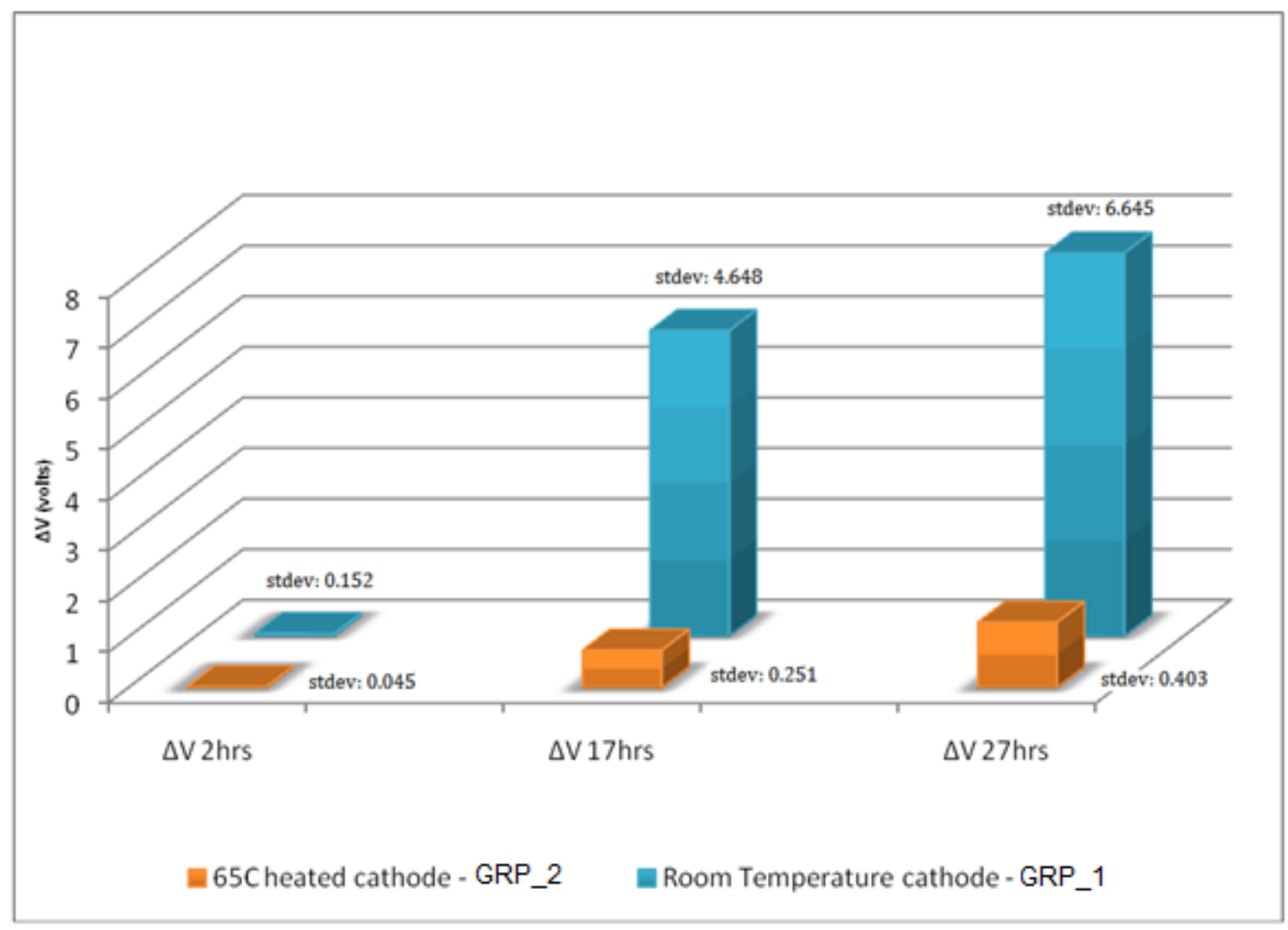

Figure 3.3: $\Delta \mathrm{V}$ graph showing voltage changes after $2,17,27 \mathrm{hrs}$ exposure to $100 \% \mathrm{RH}$ conditions for GRP_2 and GRP_1 devices

Before further analysis of the reason behind the suppression of dark spots in case of $65^{\circ} \mathrm{C}$ deposited cathode devices, a comparison of annealing(i.e. post-deposition OLED heating) and high temperature deposition is conducted to isolate any improvements or possible similarities. As stated before, prior research has provided evidence of dark spot retardation by mild annealing(i.e. post deposition heating) of the entire OLED at $70^{\circ} \mathrm{C}$ for $7.5 \mathrm{mins}$ [52]. Since we have selected metal deposition temperature to be $65^{\circ} \mathrm{C}$, the annealing temperature is also set at $65^{\circ} \mathrm{C}$ for the comparative investigation. A new set of OLEDs are fabricated (GRP_3) with the exact structure of GRP_1 OLEDs. However, after deposition, the OLEDs are annealed at $65^{\circ} \mathrm{C}$ for $40 \mathrm{mins}$ ( \pm 5 mins) inside the fabrication chamber itself, under a base pressure of 5xe-6 Torr. After annealing(i.e. post-deposition OLED heating), these OLEDs are subjected to the same controlled moisture conditions (100\%RH in air) as GRP_1 and GRP_2 devices. 
65C deposited cathode OLEDs
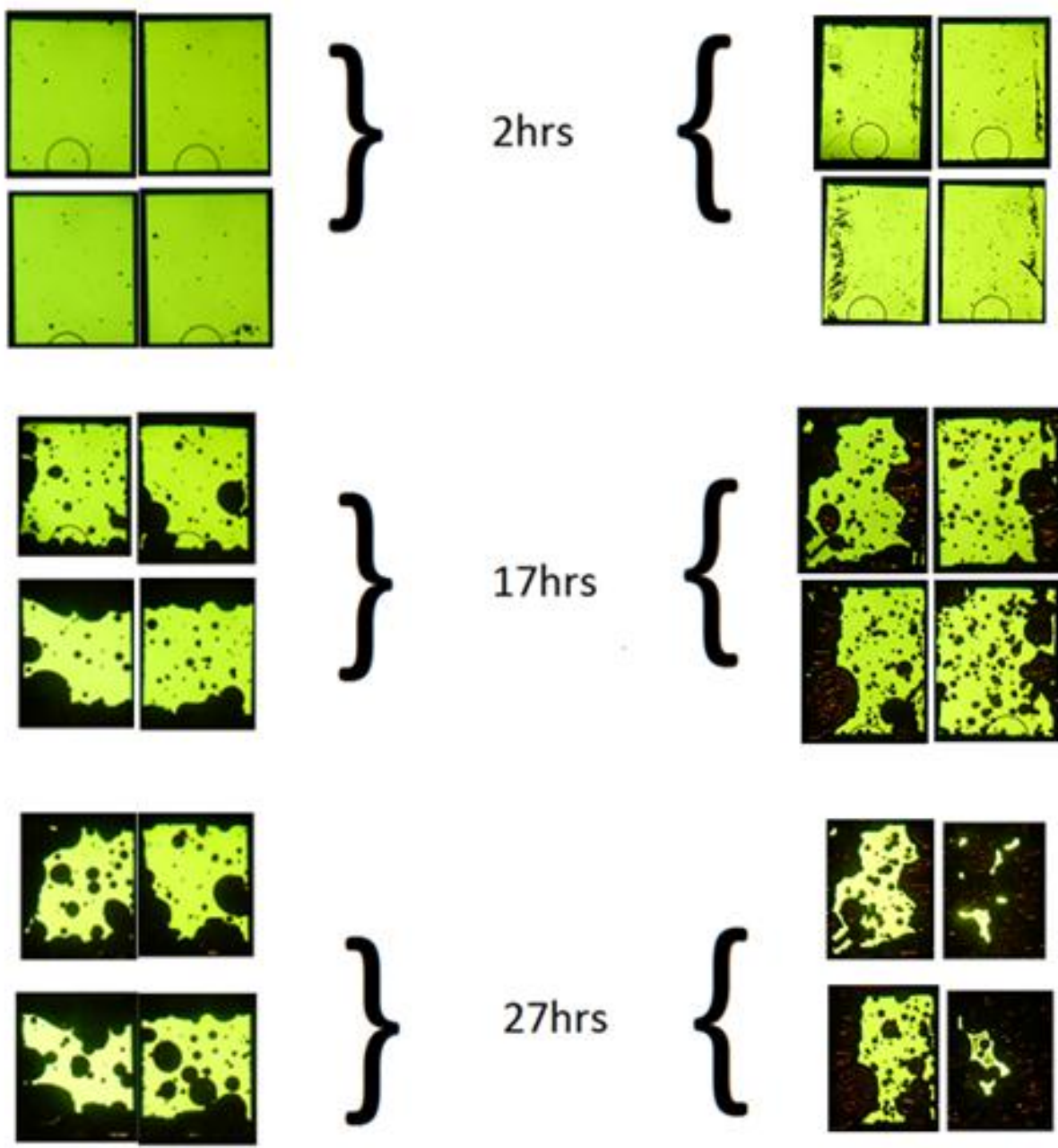

$2 \mathrm{hrs}$

$17 \mathrm{hrs}$
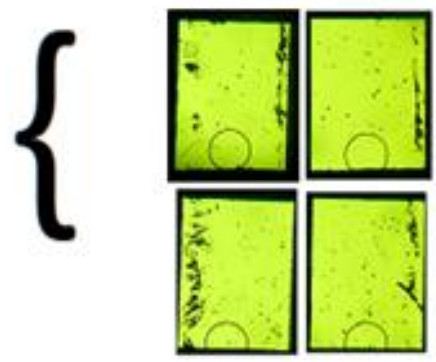

\section{C post-}

deposition

annealed OLEDs
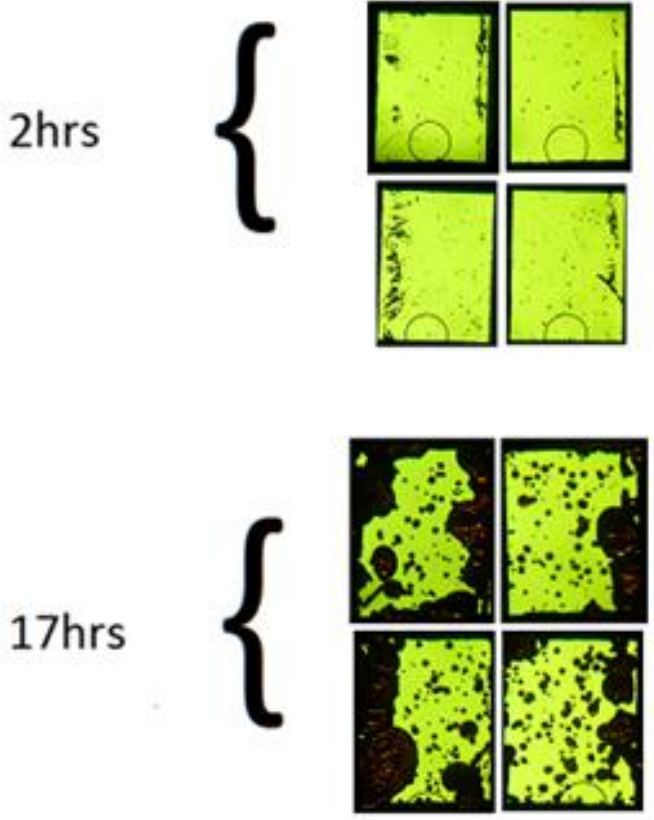

b)

a)

Figure 3.4: Dark spot growth for GRP_2 (a) vs. GRP_3 (b) at 2,17, and 27hours exposure to $100 \%$ RH conditions.

As can be seen in Fig 3.4, dark spot growth in GRP_3 annealed devices is slightly worse than the $65^{\circ} \mathrm{C}$ deposited cathode devices. Image analysis of GRP_3 micrographs after 27 hours of $100 \% \mathrm{RH}$ exposure shows that dark spot coverage ranges from $48 \%-85 \%$ of the total OLED 
surface area. On average, the OLED emissive area still functional after 27 hours of moisture exposure for GRP_3 devices is only 35\% compared to $52.75 \%$ for GRP_2 devices. Therefore a marginal improvement is observed in regards to dark spot growth suppression for $65^{\circ} \mathrm{C}$ deposited cathode OLEDs when compared to $65^{\circ} \mathrm{C}$ annealed OLEDs. $\triangle \mathrm{V}$ values are recorded for GRP_3 devices at 0,2,17,27hrs of moisture exposure as done with GRP_1 and GRP_2 devices. Figure 3.5 shows the $\Delta \mathrm{V}$ comparison for GRP_2 and GRP_3 devices, and it is seen that GRP_3 devices have a larger $\Delta \mathrm{V}$ as moisture exposure time progresses. At 27 hours of $100 \%$ moisture exposure, GRP_3 devices show an average $\Delta \mathrm{V}$ of $2.757 \mathrm{~V}$ which is a larger voltage change compared to the average GRP_2 $\Delta \mathrm{V}$ of 1.288V. This agrees with the slightly faster dark spot growth seen in Fig 3.4. This could suggest that the cathode-organic interfacial adhesion may be weaker in case of annealing the OLEDs(i.e. post-deposition OLED heating) relative to OLEDs with $65^{\circ} \mathrm{C}$ deposited cathode. It can be speculated that interfacial changes induced by annealing result in an interfacial structure with lesser adhesion that one achieved by elevated temperature cathode deposition. This assumption will be explored further by observing morphological and adhesion changes at the cathode-organic interface in the upcoming sections. 


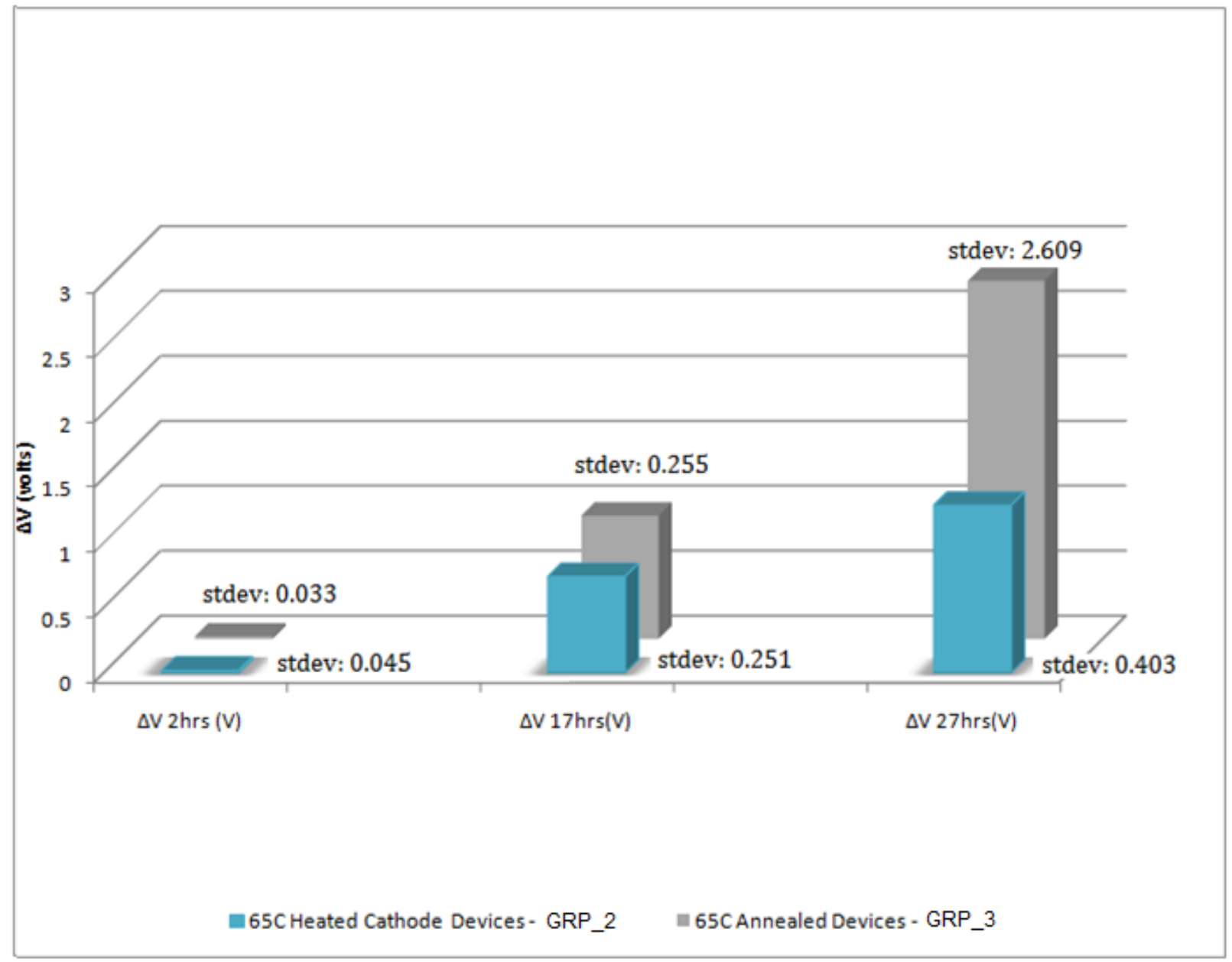

Figure 3.5: $\Delta \mathrm{V}$ graph showing voltage changes after $2,17,27 \mathrm{hrs}$ exposure to $100 \% \mathrm{RH}$ conditions for GRP_2 and GRP_3 devices

\subsection{Reasons for dark-spot suppression}

In general, slower dark spot growth can be attributed to one of the following reasons: 1)lower adsorbed residual moisture in the substrate as a result of heating, 2)morphological changes in the organic layers and/or cathode layer as a result of heating, 3)stronger adhesion between the cathode/organic layers. Therefore, we conduct a series of experiments to see which of these possibly dominates in dark spot growth suppression. 


\subsubsection{Testing for possible residual moisture loss effects}

The effect of heating the bare ITO substrate is investigated due to the potential consequence of lowering residual moisture in the substrate. In this work, the OLEDs are deposited on top of a pre-patterned ITO substrate. This substrate is heated to a temperature of $65^{\circ} \mathrm{C}$ for $30 \mathrm{mins}$ after which the organic and cathode layers are deposited via thermal deposition at room temperature. Comparing the voltage changes across time and dark spot growth of this device as compared to GRP_1 devices, there is no effective change seen. Dark spot growth and $\Delta \mathrm{V}$ changes prove to be near identical for the pre-heated ITO substrate devices and GRP_1 devices(not shown in this thesis). Hence, it can be concluded that dryness of the substrate is not a significant factor in these series of experiments relating with dark spot retardation.

\subsubsection{Testing for possible heat induced bare-ITO morphological changes}

Since the main focus of this thesis is on the cathode-organic interface, it becomes imperative to eliminate any possibility of heat-induced changes at the anode-organic interface. Even though the temperatures in this research are maintained far below the $T_{\mathrm{g}}$ of the HTL (NPB), it has yet to be established whether the anode(ITO) could potentially be the locus for heat induced change. Thus, the effect of heat on ITO(anode) is monitored to establish its role, if any, in dark spot retardation. Atomic Force Microscopy(AFM) measurements of the bare ITO substrate are taken and compared to AFM measurements of a $65^{\circ} \mathrm{C}$ heated (30min soak time) ITO substrate. These measurements are conducted to detect any superficial morphological changes as a result of direct heating of the ITO substrate. There are no effective changes in morphology observed - the RMS roughness values are similar ( $\sim 3.7 \mathrm{~nm}$ RMS roughness with comparable Z-ranges) for both the non-heat treated ITO substrate and the $65^{\circ} \mathrm{C}$ heated ITO substrate.

Hence, the slower dark spot growth in the heat treated OLEDs in this thesis cannot be attributed to morphological changes in the ITO surface. Therefore, by ruling out the role of the anode in dark spot retardation, we can focus on the heat induced changes seen on the cathode/at the cathode-organic interface in the OLEDs and their role in dark spot suppression. 


\subsubsection{Testing for possible morphological changes in the cathode, and at the cathode/organic interface}

Since residual moisture loss and ITO morphological changes have been ruled out as causative agents in dark spot suppression, further examination of the cathode-organic interfacial changes is required. Initially, the top of the cathode layer (top of the Ag layer) is scanned by AFM. This is to analyze if the changes in dark spot growth as seen in $65^{\circ} \mathrm{C}$ deposited cathode OLEDs are associated with changes in metal film morphology as a result of high temperature deposition. Fig 3.6 shows the AFM scans on top of the cathode for GRP_1 and GRP_2 devices. As can be seen from the figure, there are no morphological changes between GRP_1 and GRP_2 devices. The morphological roughness measurements give comparable RMS roughness values in the range of $3.54 \mathrm{~nm}-3.75 \mathrm{~nm}$ for GRP_1, and 3.643.91nm for GRP_2. These comparable roughness values along with the comparable Z-range values are listed in Table 3.1.

Although the above results reveal that the slower growth of dark spots in case of OLEDs with cathodes deposited at $65^{\circ} \mathrm{C}$ is not due to morphological changes in the metal cathode, it is possible that the underlying organic layers undergo some heat-induced morphological changes. In this case it can be potentially possible that the cathode is masking any morphological changes in the underlying organic layer. This "masking" behavior was observed in literature with the case of $\mathrm{Alq}_{3}$ and $\mathrm{NPB}$, where $\mathrm{Alq}_{3}$ was shown to mask the morphological changes in NPB under certain conditions[57]. Therefore, any morphological change in the underlying organic layers as a result of depositing a high temperature cathode cannot be ruled out. To investigate any heat-induced morphological changes at the cathode-organic interface, access to the interface is essential. Hence, the cathode is peeled off with 3M scotch tape, and AFM scans are conducted on the underlying organic $\left(\mathrm{Alq}_{3}\right)$ layer. Figure 3.7 shows the AFM morphological scans of top of the organic layer $\left(\mathrm{Alq}_{3}\right)$ for GRP_1 and GRP_2 devices. This figure indicates that there is no substantial morphological difference seen on top of the organic layer for GRP_1 and GRP_2 devices. GRP_1 $\mathrm{Alq}_{3} \mathrm{RMS}$ roughness ranges around 2.70$2.90 \mathrm{~nm}$ which is comparable to GRP_2 $\mathrm{Alq}_{3}$ RMS roughness of $2.66-2.79 \mathrm{~nm}$. These comparable roughness along with the comparable Z-range values are listed in Table 3.2. 
These observations do not reflect any improvement in morphology at the cathodeorganic interface due to substrate heating while cathode deposition. Hence, interfacial morphological change cannot be the primary reason behind dark spot retardation as a result of depositing the cathode at an elevated temperature.

RT cathode

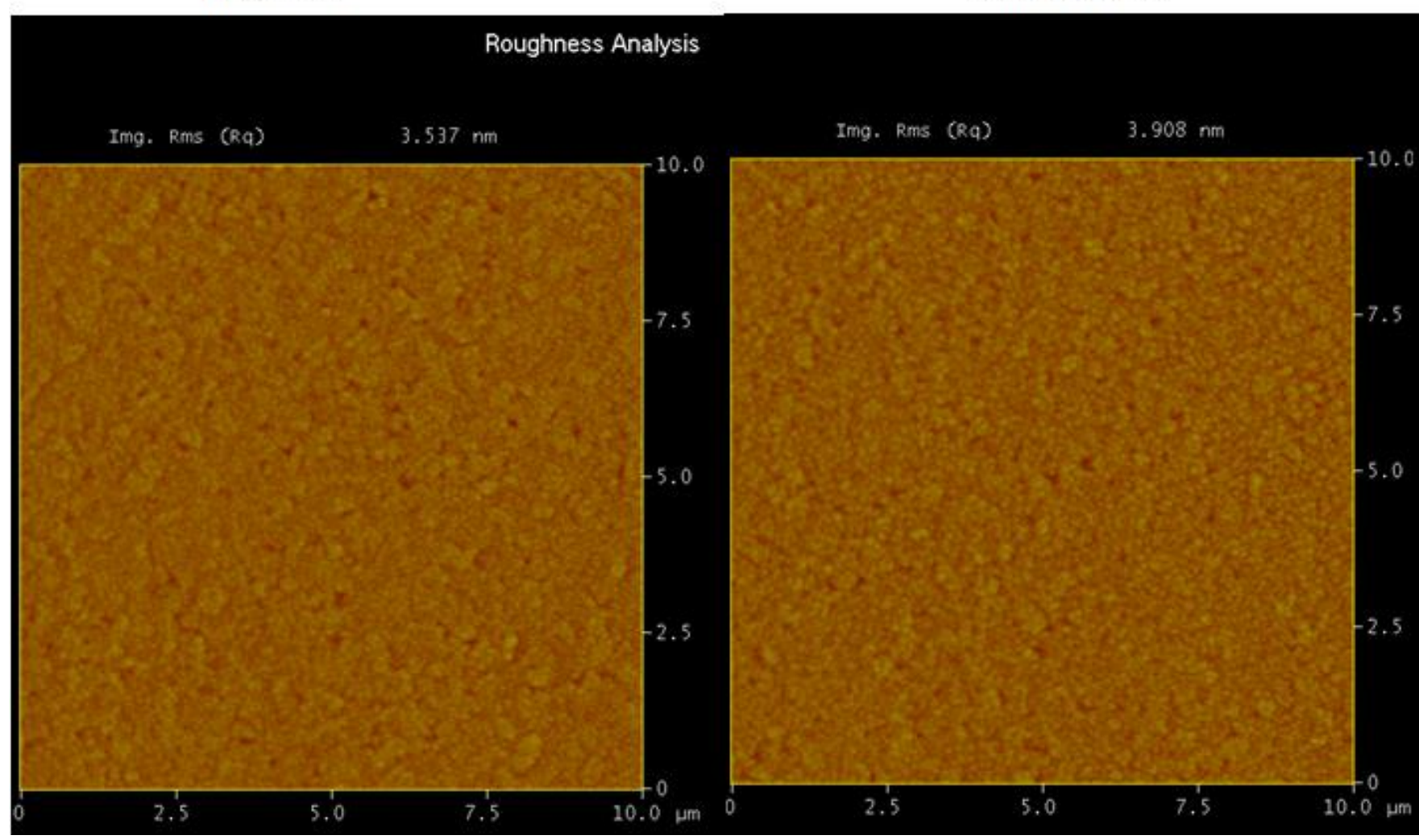

Figure 3.6: AFM Scans of the top of the cathode for GRP_1 (RT cathode) and GRP_2 $\left(65^{\circ} \mathrm{C}\right.$ deposited cathode) devices

Table 3.1 AFM roughness measurements for the top of the cathode layer

\begin{tabular}{|c|c|c|}
\hline \multicolumn{3}{|c|}{ Top of cathode (Ag) scans } \\
\hline & RMS(nm) & Z-range $(\mathrm{nm})$ \\
\hline RT cathode OLED & $3.537-3.754$ & $37.231-42.417$ \\
\hline $65^{\circ} \mathrm{C}$ deposited cathode OLED & $3.643-3.908$ & $38.794-41.877$ \\
\hline
\end{tabular}




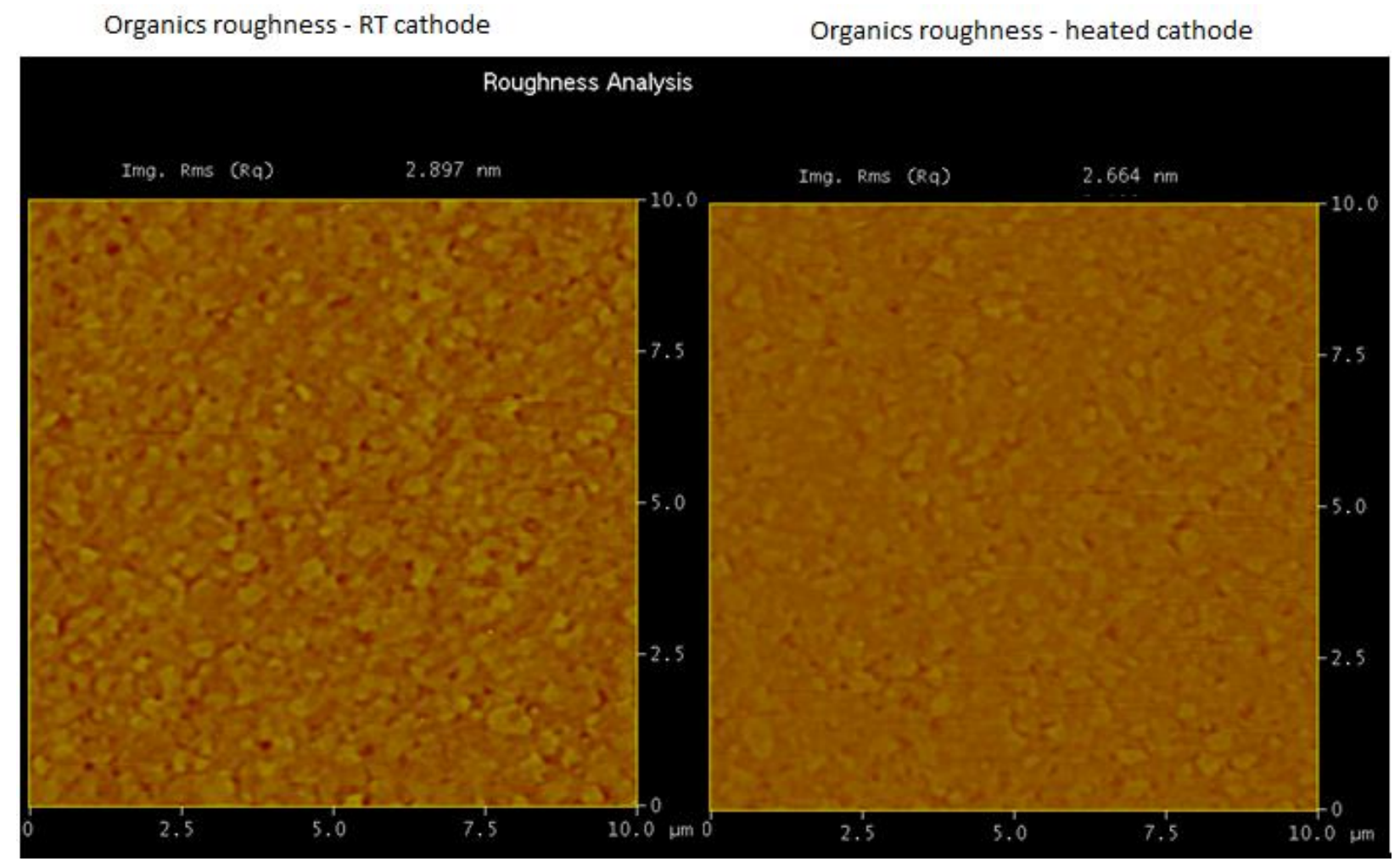

Figure 3.7: AFM Scans of the top of the organic $\left(\mathrm{Alq}_{3}\right)$ layer for GRP_1 (RT cathode) and GRP_2 $\left(65^{\circ} \mathrm{C}\right.$ deposited cathode $)$ devices

Table 3.2 AFM roughness measurements for the top of the organic layer underlying the cathode

\begin{tabular}{|c|c|c|}
\hline \multicolumn{3}{|c|}{ Top of organic material $\left(\mathrm{Alq}_{3}\right)$ scans } \\
\hline & RMS(nm) & Z-range(nm) \\
\hline RT cathode OLED & $2.699-2.897$ & $34.158-38.666$ \\
\hline $65^{\circ} \mathrm{C}$ deposited cathode OLED & $2.664-2.787$ & $33.587-39.659$ \\
\hline
\end{tabular}




\subsection{Adhesion forces at the cathode-organic interface}

Since residual moisture loss from the substrate, morphological changes at the anode, the cathode, and cathode-organic interface have been eliminated as primary reasons behind dark spot suppression in $65^{\circ} \mathrm{C}$ deposited cathode OLEDs, the speculation that $65^{\circ} \mathrm{C}$ deposited metal could result in stronger adhesion between the cathode and organic layers becomes more substantiated.

To investigate cathode-organic interfacial adhesion, three types of samples are fabricated under the same experimental conditions as the GRP_1 OLEDs. The first set of samples (S_1) consist of $\mathrm{Alq}_{3}(70 \mathrm{~nm}) / \mathrm{Mg}: \mathrm{Ag}(100 \mathrm{~nm}) / \mathrm{Mg}(100 \mathrm{~nm})$ all deposited at room temperature. The second set of samples (S_2) consist of $\mathrm{Alq}_{3}(70 \mathrm{~nm}) / 65^{\circ} \mathrm{C}$ deposited $\mathrm{Mg}: \mathrm{Ag}(100 \mathrm{~nm}) / 65^{\circ} \mathrm{C}$ deposited Ag (100nm). The third set (S_3)have the same structure as the first set, but they are annealed(i.e. post-deposition heating) at $65^{\circ} \mathrm{C}$ for 40 minutes. Once the organic and metal layers are deposited, epoxy glue (Lepage Speed Set epoxy) is applied on top of the $\mathrm{Mg}: \mathrm{Ag} / \mathrm{Ag}$ surface and a clean glass substrate is glued on top of the cathode. This is done for all the samples (S_1, S_2, S_3). The general structure of the samples after the epoxy application can be seen in Fig 3.8.

The samples are subjected to a four-point bend test in a home-built tester as described in section 2.2. The fracture energy release $\operatorname{rates}(\mathrm{G})$ are calculated from the bending tests for crack propagation at the weakest interface - the $\mathrm{Alq}_{3}-\mathrm{Mg}: \mathrm{Ag}$ interface. This is known to be the weakest interface, because when the OLED is subject to a scotch-tape peel-off test, the cathode lifts off easily, leaving behind the $\mathrm{Alq}_{3}$ layer on the substrate. From the $\mathrm{G}$ values, the degree of interfacial adhesion can be analyzed. The higher the $\mathrm{G}$ value, the stronger the interfacial adhesion.

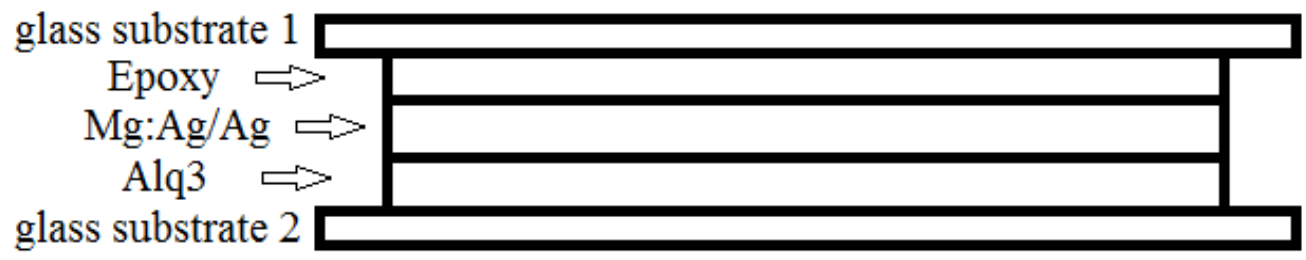

Figure 3.8: General structure of $S \_1, S \_2, S \_3$ samples 
Table 3.3 shows the $G$ values for $S \_1, S \_2$, and $S \_3$ samples. S_2 samples show an average $G$ value of $0.84 \mathrm{~J} / \mathrm{m}^{2}$ which is twice the $G$ value of $S \_1$ samples(average: $0.39 \mathrm{~J} / \mathrm{m}^{2}$ ). This suggests that cathode-organic interfacial adhesion is twice as strong for $65^{\circ} \mathrm{C}$ deposited cathode OLEDs. This could be speculated to be a result of "fusing" of the metal at the cathodeorganic interface resulting in a more adherent interface. This could be the reason behind the suppressed dark spot growth, as stronger interfacial adhesion could combat cathode delamination.

It becomes interesting now to compare the adhesion strengths of the cathode organic interface as a result of a $65^{\circ} \mathrm{C}$ deposited cathode versus $65^{\circ} \mathrm{C}$ annealing. It can be seen from Table 3.3 that the average G-value for $\mathrm{S} \_3$ structures (annealed samples at $65^{\circ} \mathrm{C}$ ) is $0.74 \mathrm{~J} / \mathrm{m}^{2}$ which is slightly lesser than that for $\mathrm{S} \_2 \operatorname{samples}\left(0.84 \mathrm{~J} / \mathrm{m}^{2}\right)$, however the difference is not big. This observation agrees with prior dark spot growth results and $\Delta \mathrm{V}$ results for GRP_2 and GRP_3 OLEDs - GRP_3 OLEDs which were the annealed OLEDs showed slightly stronger dark spot growth and slightly larger $\Delta \mathrm{V}$ values. This indicates that cathode-organic interfacial adhesion may be slightly weaker for the $65^{\circ} \mathrm{C}$ annealed OLEDs when compared to $65^{\circ} \mathrm{C}$ deposited cathode OLEDs. Perhaps cathode-organic interfacial adhesion increases differently in annealed OLEDs due to the fact that primary heating takes place after the basic interface has already been formed and the primary adhesion mechanisms have already been established. It is important to observe that $\mathrm{S} \_3$ samples are still approximately 1.8 times as adherent at the cathode-organic interface as S_1 samples. So the positive effect of annealing on the cathodeorganic interfacial adhesion certainly exists albeit to a slightly lesser degree than seen with heated-cathode deposition. Therefore, it can be stated that $65^{\circ} \mathrm{C}$ cathode deposition in an OLED results in stronger cathode-organic interfacial adhesion between the organic and cathode layers than $65^{\circ} \mathrm{C}$ annealed OLED or room temperature fabricated OLEDs. Hence, it can be concluded that cathode delamination and dark spot growth can be suppressed via strengthening the cathode-organic interfacial adhesion. 
Table 3.3 Energy release rates at the cathode-organic interface for S_1,S_2,S_3 samples

\begin{tabular}{|c|c|c|c|c|}
\hline Label & $\begin{array}{c}\text { Temperature } \\
\text { when running } \\
\text { test }\left({ }^{\circ} \mathbf{C}\right)\end{array}$ & $\begin{array}{c}\text { Humidity when } \\
\text { running } \\
\text { test }(\% \mathbf{R H})\end{array}$ & $\begin{array}{c}\text { Structure and Processing } \\
\text { Temperature }\end{array}$ & G-value $(\mathbf{J} / \mathbf{m} 2)$ \\
\hline $\mathrm{S} \_1$ & $21-22$ & $33-35$ & {$[\mathrm{Alq} / \mathrm{Mg}: \mathrm{Ag} / \mathrm{Ag}]$ at $\mathrm{RT}$} & $0.3940-0.3942$ \\
\hline $\mathrm{S} \_2$ & $22-23$ & $33-36$ & {$\left[\mathrm{Alq}_{3} / 65^{\circ} \mathrm{C}\right.$ deposited $\left.\mathrm{Mg}: \mathrm{Ag} / \mathrm{Ag}\right]$} & $0.8339-0.8467$ \\
\hline S_3 & 24 & 40 & {$\left[\mathrm{Alq}_{3} / \mathrm{Mg}: \mathrm{Ag} / \mathrm{Ag}\right]$ annealed at $65^{\circ} \mathrm{C}$} & $0.7189-0.7610$ \\
\hline
\end{tabular}




\section{Chapter 4 Effect of using cathode-organic interfacial layers on dark spot growth and cathode-organic interfacial adhesion}

Finding that cathode-organic interfacial adhesion improves via $65^{\circ} \mathrm{C}$ cathode deposition and results in dark spot suppression, it becomes interesting to see if the use of interfacial layers between the cathode and organic layers could also enhance adhesion, and hence slow down the growth of dark spots. The use of intermediate layers to "grade" the interface presents a way of having a gradual cathode/organic interface instead of an abrupt interface. Grading the interface may have the potential to increase the adhesive forces at the interface by increasing the effective surface area. The mixed organic metal interfacial layer(MOML) used by Aziz et al [53] has showed potential for dark spot suppression at 24 hours in ambient conditions. In this work, the exact MOML composition as used by Aziz et al[53] is utilized for the cathode-organic interfacial layer.

\subsection{Effect of using cathode-organic interfacial MOML on dark spot growth}

A new group of OLEDs(GRP_4) are fabricated with the structure seen in Fig 4.1. A mixture of $\mathrm{Alq}_{3}: \mathrm{Mg}: \mathrm{Ag}(47.5: 47.5: 5 \%$ by volume) [53] is used as the interfacial layer between the strict $\mathrm{Alq}_{3}$ layer and the $\mathrm{Mg}: \mathrm{Ag}$ cathode layer. The deposition rates for this mixed layer are 1.8,1.8,0.2 A /s respectively. The MOML thickness is approximately 310nm. After 310nm of MOML deposition, the MOML is tapered off into the cathode $\left(\mathrm{Alq}_{3}\right.$ deposition is stopped, while the Mg:Ag deposition is continued).GRP_4 OLEDs are fabricated under a base pressure of $5 \times 10^{-6}$ Torr and via thermal deposition just like GRP_1 and GRP_2 devices. The fabrication is carried out entirely at room temperature(RT) without substrate heating or post-deposition heating. GRP_4 devices are subjected to similar moisture exposure conditions as seen with GRP_1 and GRP_2 devices. 


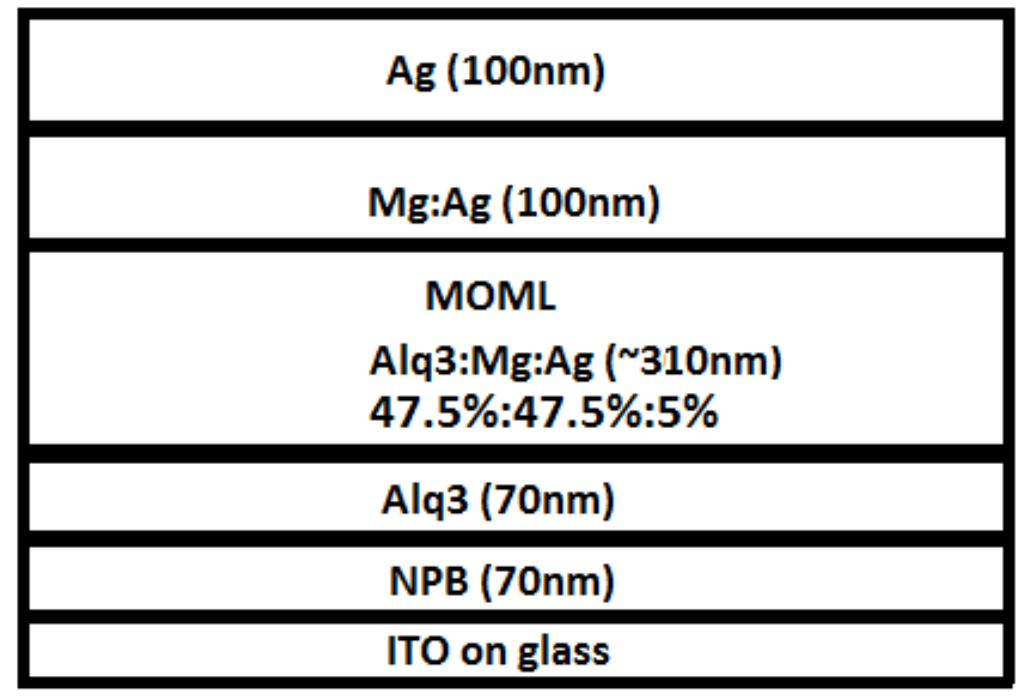

Figure 4.1: Structure of an OLED with a cathode-organic interfacial MOML

As expected, dark spot growth suppression is observed with GRP_4 OLEDs. Fig 4.2 shows dark spot growth micrographs at 2,17/20,27hrs of exposure to100\%RH for GRP_1 and GRP_4 devices respectively. As observed with GRP_2 devices, dark spot growth at 2 hours 100\% RH exposure for GRP_4 devices is lesser than seen win GRP_1. At 27 hours of moisture exposure, the dark spot suppression is apparent $-77.7 \%$ of the total surface area of the OLED is still emissive for GRP_4 devices compared to only $2 \%$ functional emissive area for GRP_1 OLEDs. In general, GRP_4 devices should be less resistive than GRP_1 devices as they have minimal dark spot emissive area coverage. $\Delta \mathrm{V}$ measurements recorded at 2,20/17,27 hours for GRP_4 and GRP_1 devices, as seen in Fig 4.3, agree with the observations seen in the dark spot micrographs. As expected, the average $\Delta \mathrm{V}$ for GRP_4 is much lower than GRP_1 devices - at 27 hrs exposure to $100 \% \mathrm{RH}, \Delta \mathrm{V}$ for GRP_4 and GRP_1 are $2.726 \mathrm{~V}$ and $7.568 \mathrm{~V}$ and respectively. These results reveal that using a graded interfacial layer may result in stronger cathode-organic interfacial adhesion. Further examination of the cathode-organic interface is necessary to investigate whether interfacial adhesion increases as a result of using a MOML. 
Room

Temperature(RT) deposited OLEDs
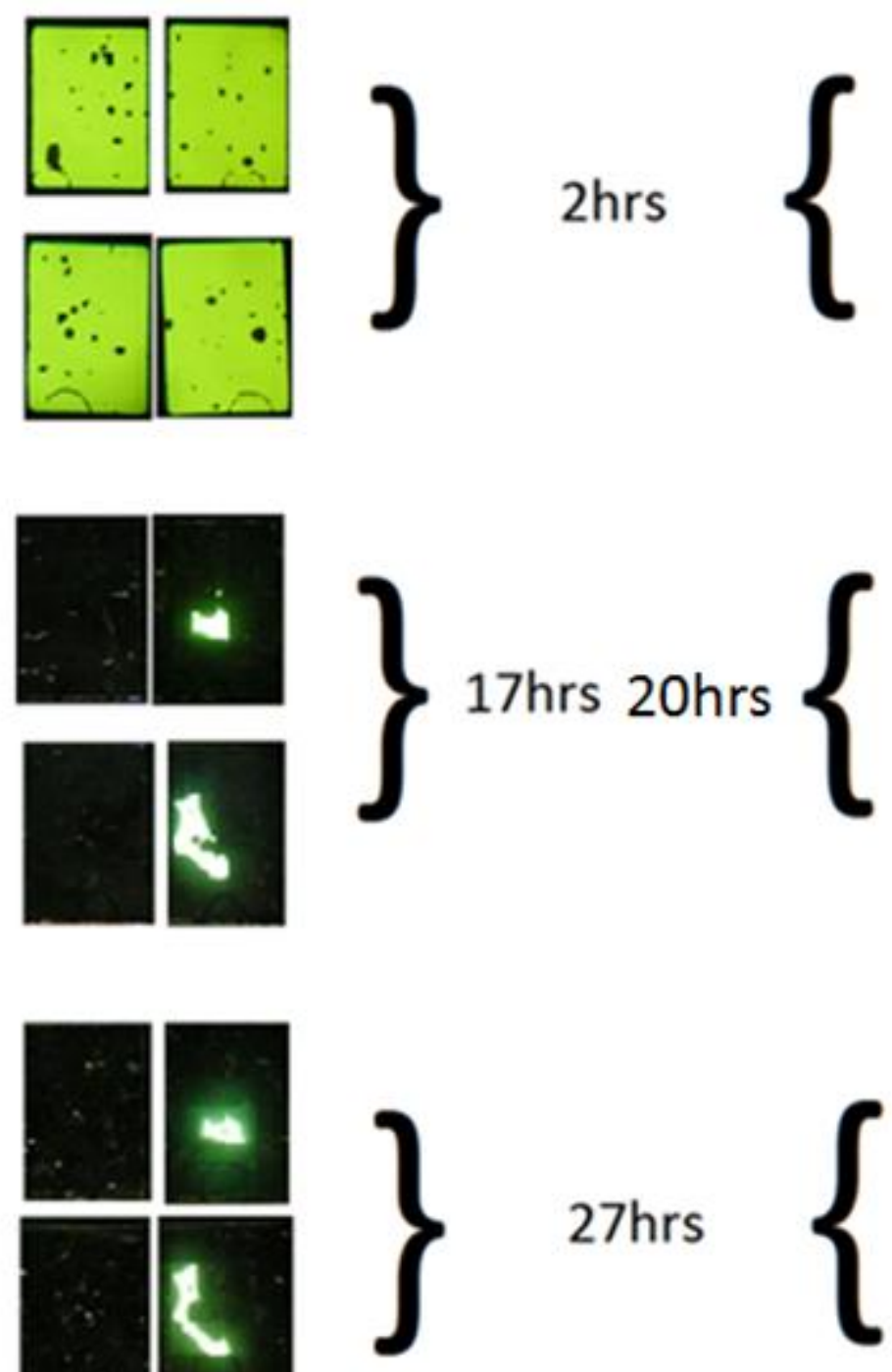

27hrs a)

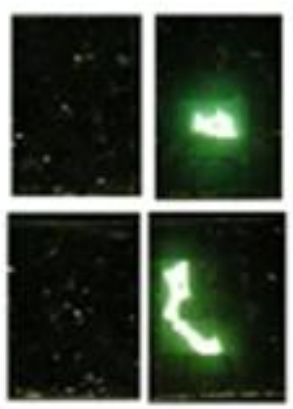

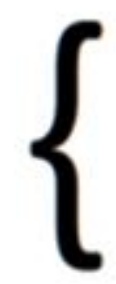

b)
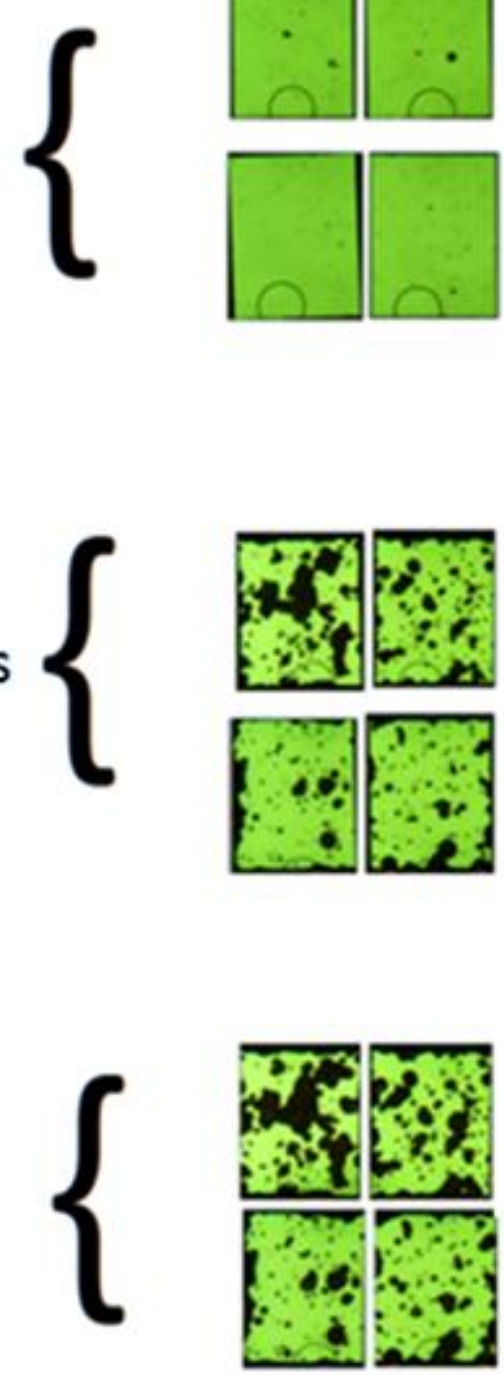

OLEDs with

cathode-organic

MOML
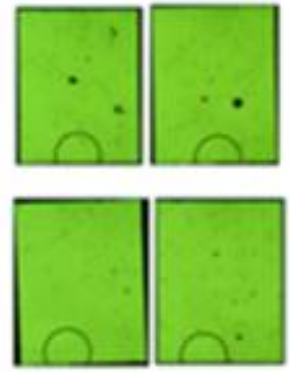

Figure 4.2: Dark spot growth for GRP_1 (a) vs. GRP_4 (b) at 2,17, and 27hours exposure to $100 \% \mathrm{RH}$ conditions. 


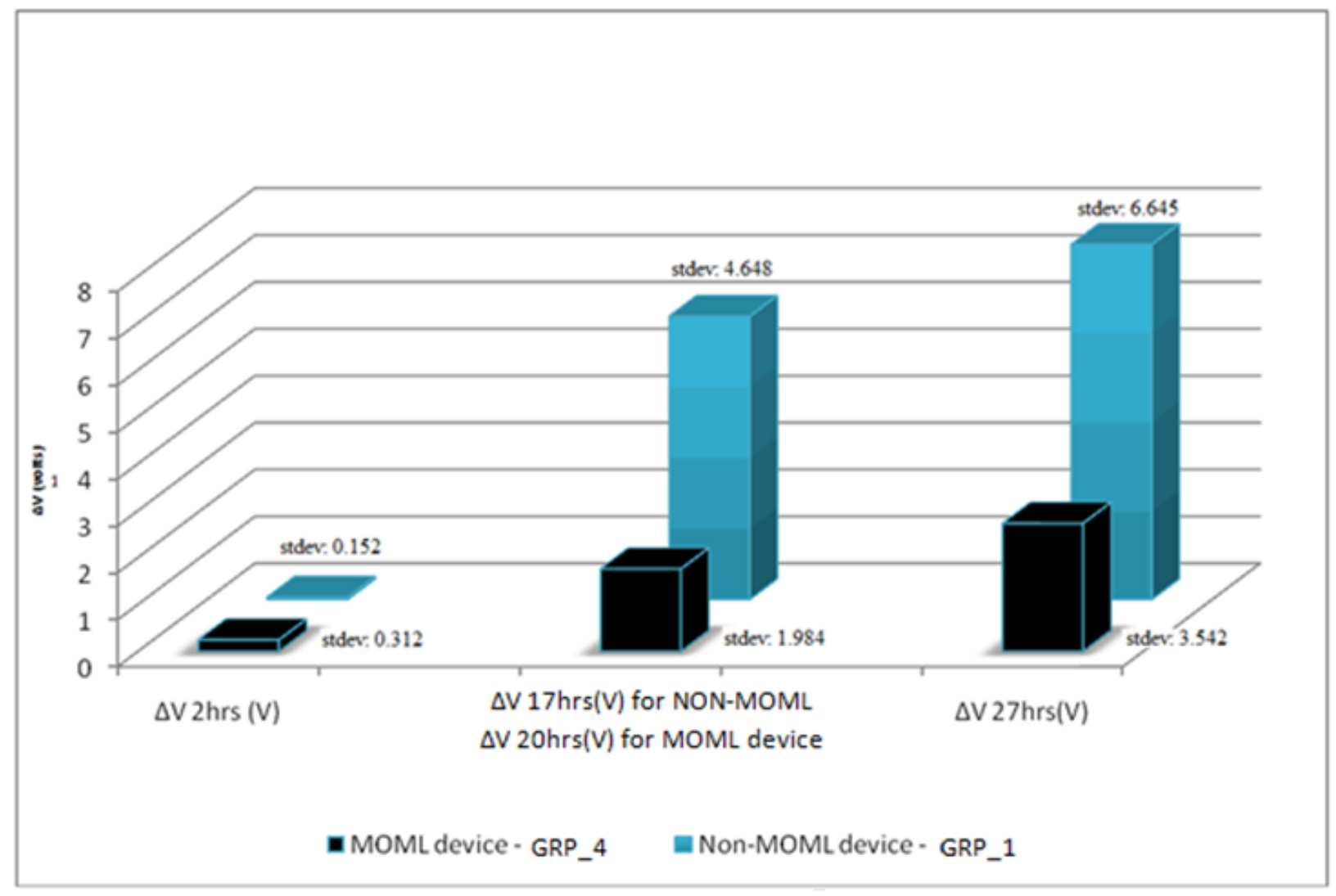

Figure 4.3: $\Delta \mathrm{V}$ graph showing voltage changes after $2,17,27 \mathrm{hrs}$ exposure to $100 \% \mathrm{RH}$ conditions for GRP_1 and GRP_4 devices

\subsection{Effect of using cathode-organic interfacial MOML on cathode-organic interfacial adhesion}

To further investigate cathode-organic interfacial adhesion with the use of a MOML interfacial layer, samples with the structure : $\mathrm{Alq}_{3}(70 \mathrm{~nm}) / \mathrm{MOML}(310 \mathrm{~nm}) / \mathrm{Mg}: \mathrm{Ag}(100 \mathrm{~nm}) / \mathrm{Ag}(100 \mathrm{~nm})$ are fabricated on top of a glass substrate. The fabrication conditions are the same as with GRP_4 OLEDs. As seen in samples S_1, S_2, and S_3, epoxy glue (Lepage Speed Set epoxy) is applied on top of the cathode and another glass substrate is glued onto the cathode forming a sandwiched structure. This structure is illustrated in Fig 4.4. S_4 samples are subjected to the same four-point bend test as S_1 
samples. The load values for fracture propagation at the weakest interface ( $\left.\mathrm{Alq}_{3}-\mathrm{MOML}\right)$ are collected and $\mathrm{G}$ values are calculated. Comparison of the $\mathrm{G}$ values for S_4 and S_1 samples, as seen in Table 4.1, indicates that cathode-organic interfacial adhesion is approximately two and a half times stronger for samples with the MOML interfacial layer(average: $0.97 \mathrm{~J} / \mathrm{m}^{2}$ ) when compared to non-MOML samples(average: $0.39 \mathrm{~J} / \mathrm{m}^{2}$ ) as seen in Table 4.1 .

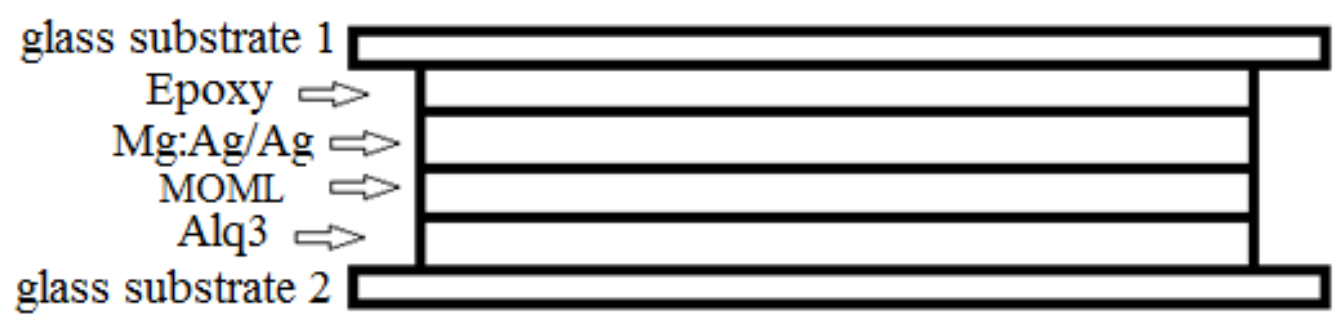

Figure 4.4: S_4 sample structure

Table 4.1 Energy release rates at the cathode-organic interface for $S \_1$ and $S \_4$ samples

\begin{tabular}{|c|c|c|c|c|}
\hline Label & $\begin{array}{c}\text { Temperature } \\
\text { when running } \\
\text { test }\left({ }^{\circ} \mathbf{C}\right)\end{array}$ & $\begin{array}{c}\text { Humidity when } \\
\text { running } \\
\text { test }(\% \mathbf{R H})\end{array}$ & $\begin{array}{c}\text { Structure and Processing } \\
\text { Temperature }\end{array}$ & $\begin{array}{c}\text { G-value } \\
\mathbf{( J / m 2})\end{array}$ \\
\hline $\mathrm{S} \_1$ & $21-22$ & $33-35$ & {$\left[\mathrm{Alq}_{3} / \mathrm{Mg}: \mathrm{Ag} / \mathrm{Ag}\right]$ at $\mathrm{RT}$} & $0.3940-0.3942$ \\
\hline $\mathrm{S} \_4$ & $23-24$ & $35-40$ & {$\left[\mathrm{Alq}_{3} / \mathrm{Alq}_{3}: \mathrm{Mg}: \mathrm{Ag} / \mathrm{Mg}: \mathrm{Ag} / \mathrm{Ag}\right]$ at $\mathrm{RT}$} & $0.9199-1.0151$ \\
\hline
\end{tabular}

These observations reveal that interfacial adhesion between the cathode and the organic layers is definitely increased as a result of using a graded interfacial layer. It is interesting to notice that a higher energy release rate is observed for S_4 samples (MOML structures) when compared to the $65^{\circ} \mathrm{C}$ heated cathode samples (S_2). This observation is in agreement with the stronger dark spot suppression seen in GRP_4 OLEDs which use the MOML. Furthermore, GRP_4 OLEDs show better dark spot suppression when compared to GRP_2 $\left(65^{\circ} \mathrm{C}\right.$ heated cathode) OLEDs, which can now be attributed to stronger interfacial adhesion between the cathode and organic layers for GRP_4 devices. These findings establish the role of interfacial adhesion in suppression of dark spot growth. 


\subsection{Effect of using abrupt vs. seamless MOML-cathode transition on dark spot growth}

As a graded interface has been shown to strengthen adhesion at the cathode/organic interface, it becomes interesting to investigate the advantageous effect, if any, of tapering the MOML into the cathode. GRP_4 OLEDs were fabricated in such a way that the MOML was tapered off into the cathode. This means that the $\mathrm{Alq}_{3}$ deposition rate was decreased from $1.8 \AA \mathrm{A} / \mathrm{s}$ to $0 \AA / \mathrm{s}$, while letting the Mg:Ag deposit at its ongoing rate of 1.8:0.2 $\mathrm{A} / \mathrm{s}$. Any advantages of this tapering effect can be isolated by comparing dark spot growth with devices fabricated with an abrupt MOML to cathode transition. An abrupt MOML would mean that the deposition of $\mathrm{Alq}_{3}$, and $\mathrm{Mg}: \mathrm{Ag}$ is stopped at the end of MOML deposition completely. After this abrupt stop, the $\mathrm{Mg}: \mathrm{Ag}$ cathode is deposited- thereby potentially inducing an abrupt MOML-Mg:Ag interface. Hence, OLEDs(GRP_5) with an abrupt MOML-Mg:Ag interface are fabricated under the same deposition conditions as GRP_4 OLEDs. Another group of OLEDs is fabricated (GRP_6) with similar deposition conditions as associated with GRP_4 OLEDs. After 27 hour 100\% moisture exposure, GRP_5 OLEDs show comparable dark spot growth as seen in GRP_6 OLEDs. Micrographs as seen in Fig 4.5 reveal that after 27 hours of 100\%RH exposure, $74.8 \%$ of GRP_5 OLED surface area is still emissive, which is comparable with the 77.0-79\% active emissive area seen with GRP_6 OLEDs. These observations are in agreement with the voltage changes seen across GRP_5 and GRP_6 OLEDs as shown in Fig 4.6. Table 4.2 shows that GRP_5 OLEDs have an average $\Delta \mathrm{V}$ of $2.55 \mathrm{~V}$ which is marginally worse than $1.39 \mathrm{~V}$ seen for GRP_6 devices. The marginal difference can be attributed to the 3-5\% increase in dark spot coverage seen in GRP_5 devices. No definite improvements are seen as a result of tapering the MOML into the cathode. This suggests that tapering of the MOML into the metal may not have a strong interfacial strengthening effect.

It should be noted that the tapering of the MOML in GRP_5 devices was carried out by significantly reducing the $\mathrm{Alq}_{3}$ rate from $1.8 \AA / \mathrm{s}$ to $0 \AA / \mathrm{s}$. So, in spite of this initial attempt to taper the MOML, the sudden decrease in the $\mathrm{Alq}_{3}$ rate might have resulted in similar interfacial effects as seen in the abrupt MOML-Mg:Ag devices(GRP_5). Further investigations into grading the MOML by decrementing the $\mathrm{Alq}_{3}$ rate from $1.8 \AA / \mathrm{A}$ gradually to $0 \AA \mathrm{A} / \mathrm{s}$ may be beneficial. 


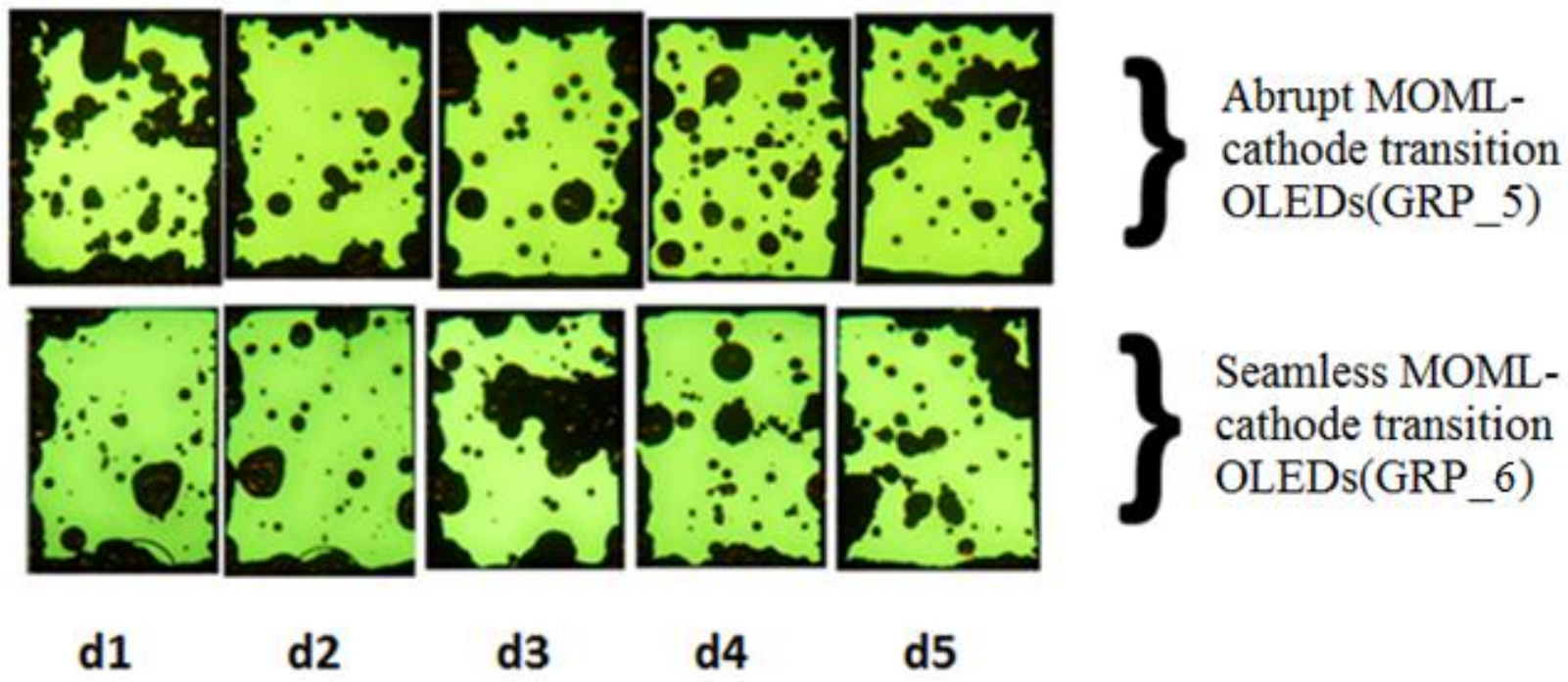

Figure 4.5: Dark spot growth after 27 hour exposure to 100\% RH conditions for abrupt(GRP_5) vs. seamless MOML $\rightarrow$ cathode transition (GRP_6) OLEDs. 


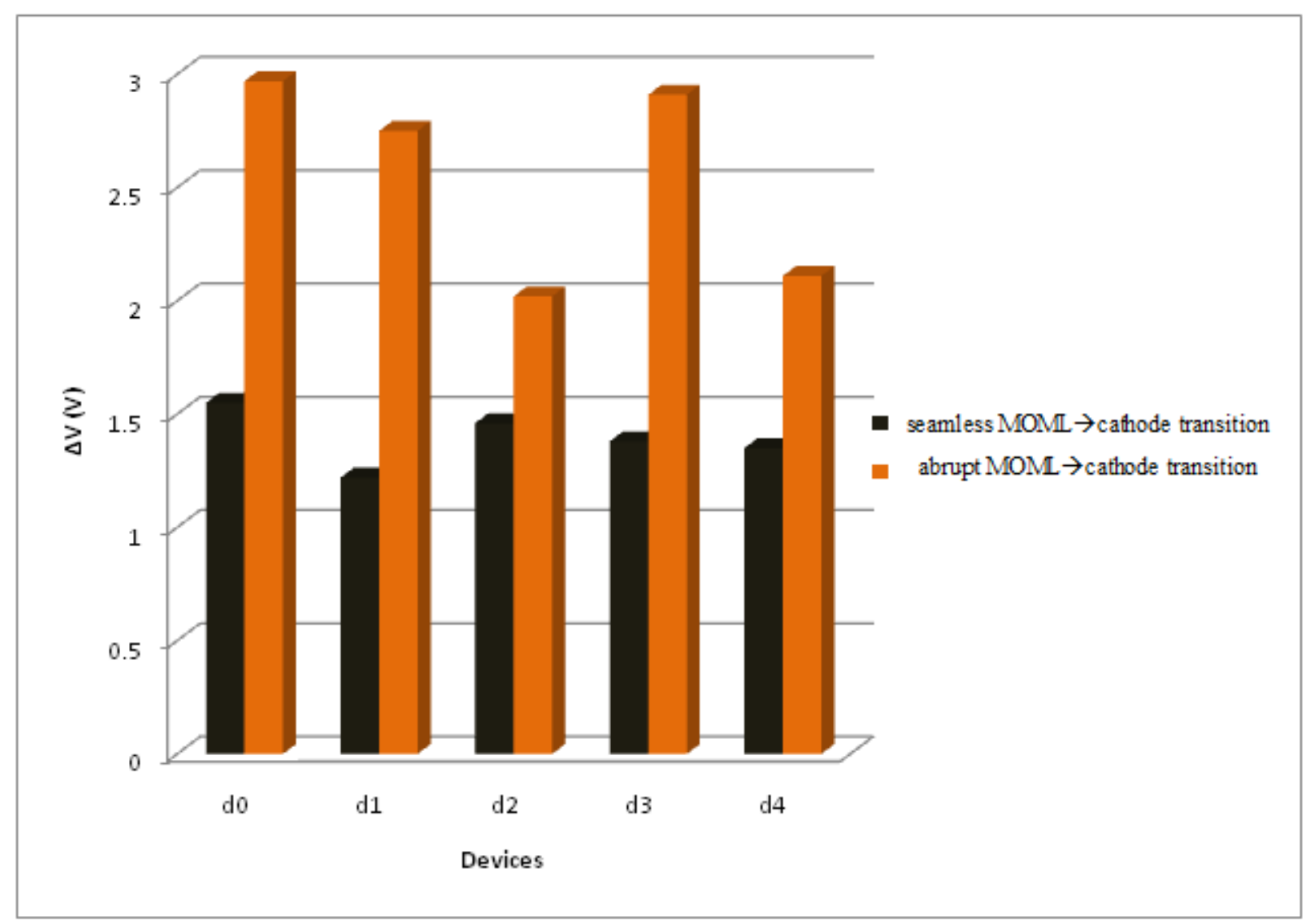

Figure 4.6: $\Delta \mathrm{V}$ graph for GRP_5 and GRP_6 OLEDs shown in Fig 3.15.

Table 4.2 Average $\Delta \mathrm{V}$ after $27 \mathrm{hrs}$ exposure to $100 \% \mathrm{RH}$ conditions for seamless(GRP_6) and abrupt(GRP_5) MOML $\rightarrow$ cathode transition OLEDs

\begin{tabular}{|c|c|}
\hline Deposition & $\begin{array}{c}\text { Average } \Delta \mathrm{V} \text { after 27hrs exposure to } \\
100 \% \mathrm{RH}(\mathrm{V})\end{array}$ \\
\hline GRP_6 : seamless MOML $\rightarrow$ cathode transition & 1.392 \\
\hline GRP_5: abrupt MOML $\rightarrow$ cathode transition & 2.552 \\
\hline
\end{tabular}




\subsection{Effect of using $65^{\circ} \mathrm{C}$ deposited MOML $+65^{\circ} \mathrm{C}$ deposited cathode on dark spot growth}

Since heating the cathode while deposition, and using an interfacial MOML at the cathode-organic interface have been shown to improve interfacial adhesion separately, further examination to investigate the cumulative effect of heat and MOML on dark spot suppression is needed.

For this purpose, OLEDs(GRP_7) are fabricated in which both the MOML and the cathode are deposited at $65^{\circ} \mathrm{C}$. GRP_7 devices are subject to the same moisture conditions as used for GRP_4 OLEDs (room temperature MOML+cathode OLEDs). As seen from Fig 4.7, 27 hour exposure to $100 \%$ RH results in comparable dark spot growth for GRP_4 and GRP_7 devices. Apparently, heating the MOML and the cathode while deposition does not result in any dark spot suppression improvements when compared to the lone effect of using a MOML interfacial layer. These results suggest that the use of the MOML and the separate use of heating while cathode deposition results in improved cathode-organic interfacial adhesion, however the result is not cumulative. Any interfacial strengthening effects as a result of using a graded layer cannot be further improved by the effect of cathode or MOML heating. 


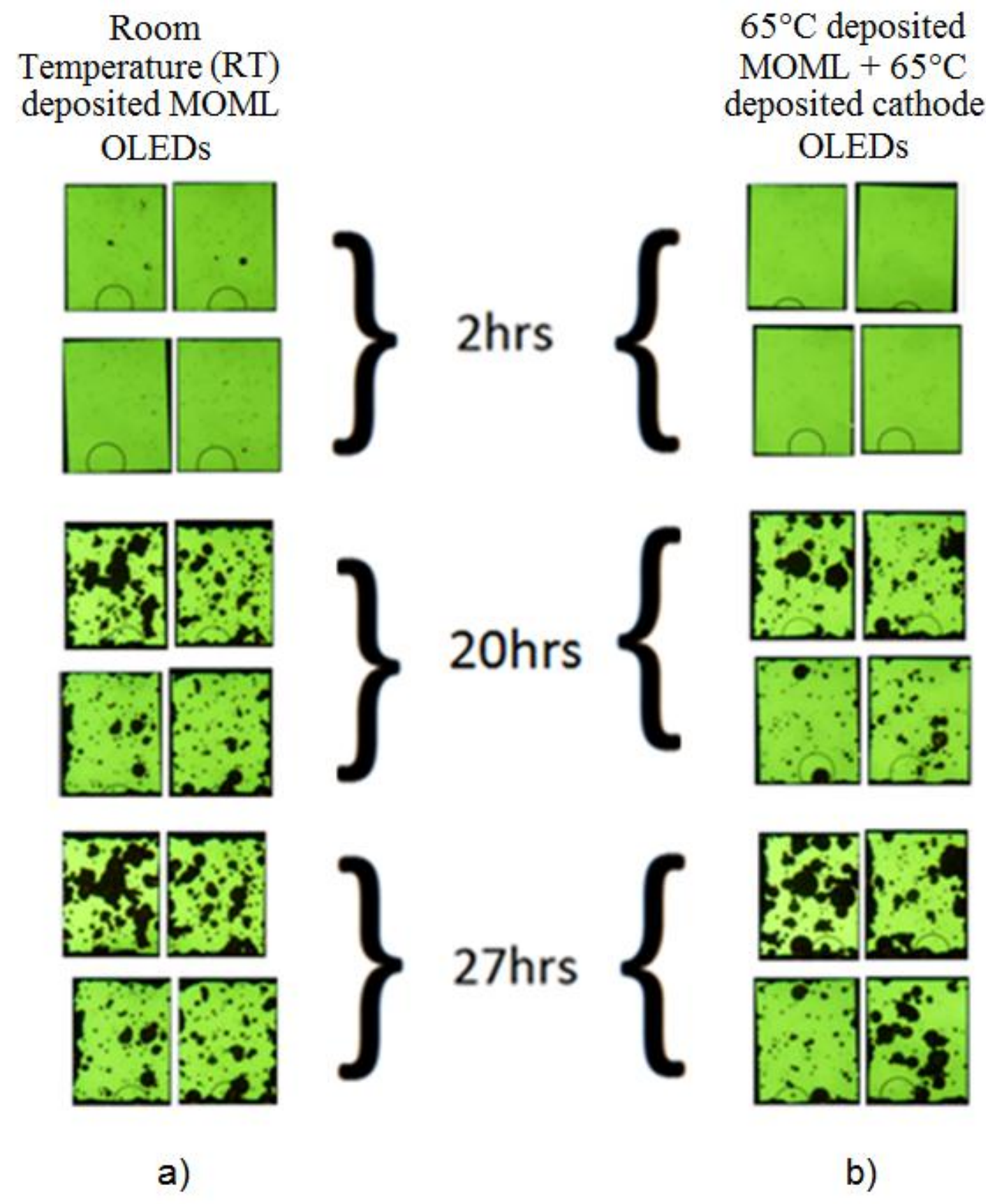

Figure 4.7: Dark spot growth for GRP_4 (a) vs. GRP_7(b) at 2,17, and 27hours exposure to $100 \%$ RH conditions. 


\subsection{Effect of using electron injection layers on cathode-organic interfacial adhesion}

Interfacial layers such as electron injection layers have been widely used in literature to improve electron injection at the cathode-organic interface. Now that we have established the advantages of an interfacial layer - such as the MOML - for dark spot retardation, the effect of conventional electron-injection layers(EIL)-like $\mathrm{LiF}$ and $\mathrm{Cs}_{2} \mathrm{CO}_{3}$ on interfacial adhesion is further investigated. OLEDs with the following structure are fabricated: ITO/NPB(70nm)/ $\mathrm{Alq}_{3}$ $(70 \mathrm{~nm}) / \mathrm{LiF}(1 \mathrm{~nm})$-or- $\mathrm{Cs}_{2} \mathrm{CO}_{3}(1 \mathrm{~nm}) / \mathrm{Mg}: \mathrm{Ag}(100 \mathrm{~nm}) / \mathrm{Ag}(100 \mathrm{~nm})$. After 22 days of storage in nitrogen, $\Delta \mathrm{V}$ for OLEDs with $\mathrm{LiF}(1.70 \mathrm{~V})$ or $\mathrm{Cs}_{2} \mathrm{CO}_{3}(1.1 \mathrm{~V})$ as EILs did not exhibit any definite $\Delta \mathrm{V}$ improvements over GRP_1 OLEDs $\Delta \mathrm{V}$ value (1.6V). In this case, it can be speculated that the use of an EIL such as $\mathrm{LiF}$ or $\mathrm{Cs}_{2} \mathrm{CO}_{3}$ leads to no significant interfacial strengthening at the cathode-organic interface .

To prove the aforementioned speculation, structures with organic layers/EIL(LiF or $\mathrm{Cs}_{2} \mathrm{CO}_{3}$ )/cathode layer (S_5 - $\mathrm{LiF}, \mathrm{S} \_6-\mathrm{Cs}_{2} \mathrm{CO}_{3}$ ) are fabricated (as seen in Fig 4.8) and are subjected to the same four point bend test as previously reported. Table 4.3 shows the various Gvalues for structures with EILs and without an EIL. As can be seen from the table, the G-values for $\mathrm{LiF}\left(\mathrm{S} \_5\right)$ and $\mathrm{Cs}_{2} \mathrm{CO}_{3}$ (S_6) are comparable and are in the range of 0.51 to $0.60 \mathrm{~J} / \mathrm{m}^{2}$ (on average). In addition, the G-values for S_5 and S_6 are marginally higher than for S_1 samples, which have a $\mathrm{G}$ value of $0.39 \mathrm{~J} / \mathrm{m}^{2}$. As the difference in G-values between the EIL and non-EIL samples is very small, it can be said that there is no definite improvement in interfacial adhesion as a result of using a $1 \mathrm{~nm}$ thick EIL at the cathode-organic interface. This lack of interfacial adhesion improvement is in accordance with the comparable $\Delta \mathrm{V}$ changes seen for the EIL and non-EIL OLEDs. However, referring to Tables 4.1 and 4.3, it is important to note that the G values for S_5 and S_6 (0.51 to $\left.0.60 \mathrm{~J} / \mathrm{m}^{2}\right)$ are much smaller than those recorded for S_4 MOML samples $\left(0.92-1.02 \mathrm{~J} / \mathrm{m}^{2}\right)$. Hence, unlike thick $(\sim 310 \mathrm{~nm})$ cathode-organic interfacial layers like a MOML, traditional $1 \mathrm{~nm}$ thick EILs such as $\mathrm{LiF}$ and $\mathrm{Cs}_{2} \mathrm{CO}_{3}$ do not induce interfacial strengthening. Even though $\mathrm{LiF}$ and $\mathrm{Cs}_{2} \mathrm{CO}_{3}$ may help with electron injection and lowering initial voltage responses for OLEDs, they might not assist in adhesion-strengthening at the cathode-organic interface. Vollmer-Weber like island structures may result with thin 1nm EIL films- thereby not forming a continuous film at the cathode-organic interface. This may be the 
reason why the $1 \mathrm{~nm}$ EILs do not induce interfacial adhesion-strengthening. Therefore, it can be concluded that the type of cathode-organic interface plays an important role in dark spot suppression. This discussion concludes the investigation of interfacial adhesion and the study of improving ambient stability by modifying the cathode-organic interface.

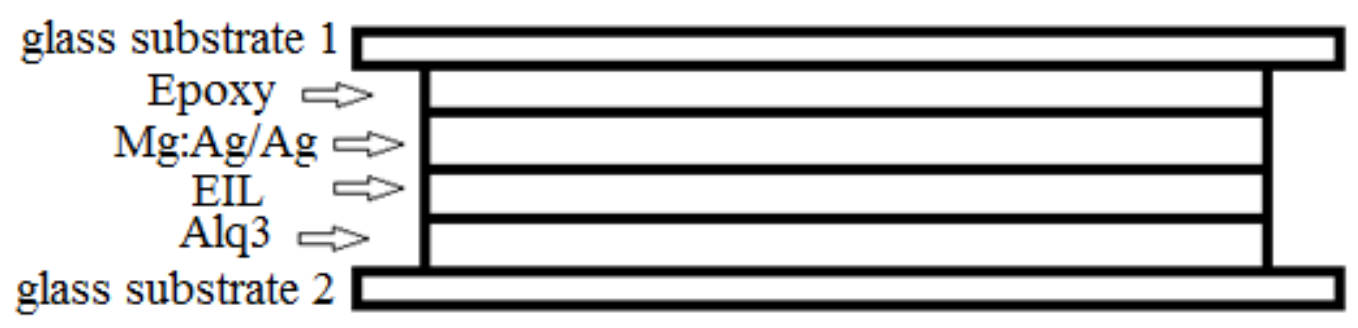

Figure 4.8: S_5/6 sample structure

Table 4.3 Energy release rates at the cathode-organic interface for samples S_1,S_5, and S_6

\begin{tabular}{|c|c|c|c|c|}
\hline Label & $\begin{array}{c}\text { Temperature range } \\
\text { when running } \\
\text { test }\left({ }^{\circ} \mathbf{C}\right)\end{array}$ & $\begin{array}{c}\text { Humidity when } \\
\text { running } \\
\text { test }(\% \mathbf{R H})\end{array}$ & $\begin{array}{c}\text { Structure and Processing } \\
\text { Temperature }\end{array}$ & G-value $(\mathbf{J} / \mathbf{m}$ 2) \\
\hline S_1 & $21-22$ & $33-35$ & {$\left[\mathrm{Alq}_{3} / \mathrm{Mg}: \mathrm{Ag} / \mathrm{Ag}\right]$ at $\mathrm{RT}$} & $0.3940-0.3942$ \\
\hline S_5 & $23-24$ & $39-41$ & {$\left[\mathrm{Alq}_{3} / \mathrm{LiF} / \mathrm{Mg}: \mathrm{Ag} / \mathrm{Ag}\right]$ at $\mathrm{RT}$} & $0.5116-0.5134$ \\
\hline S_6 & $23-24$ & $39-41$ & {$\left[\mathrm{Alq}_{3} / \mathrm{Cs}_{2} \mathrm{CO}_{3} / \mathrm{Mg}: \mathrm{Ag} / \mathrm{Ag}\right]$ at } & $0.5769-0.6167$ \\
\hline
\end{tabular}




\section{Chapter 5 Exploring novel thin-film encapsulation layers}

As mentioned before, encapsulation of OLEDs is a popular industry solution for dark spot suppression. As stated in the section 1.7, thin film encapsulation is the most effective encapsulation technique for flexible OLEDs. In this work, brief investigations into the use of Titanium (Ti) and Molybdenum Oxide $\left(\mathrm{MoO}_{3}\right)$ as part of thin film OLED encapsulation structures are discussed.

Titanium is chosen as a viable encapsulant due to its uniform film forming properties and its resistance to mechanical damage. Literature also shows that $1 \mathrm{~nm}$ thin Ti films exhibit superior corrosion resistance to salt water when subjected to a calcium test[58].

OLEDs with the structure $\mathrm{NPB}(70 \mathrm{~nm}) / \mathrm{Alq}_{3}(70 \mathrm{~nm}) / \mathrm{Mg}: \mathrm{Ag}($ varying$) / \mathrm{Ag}(100 \mathrm{~nm})$ are fabricated under the same deposition parameters as seen in GRP_1 OLEDs. A 200nm Ti layer is deposited via electron-beam(e-beam) deposition at a rate of $4 \AA / s$. The Mg:Ag layer thickness is varied across five OLEDs with thickness values of 5,10,25,50,100nm. Luminance and voltage responses recorded for an input current density of $20 \mathrm{~mA} / \mathrm{cm}^{2}$ are shown in Table 5.1. As can be seen from the table, luminous intensity decreases as the thickness of the Mg:Ag cathode decreases. The best brightness $\left(294 \mathrm{~cd} / \mathrm{m}^{2}\right)$ is seen for Mg: Ag layer thickness of $100 \mathrm{~nm}$. For comparison, GRP_1 OLEDs with a 200nm Mg:Ag/Ag cathode have an average luminance output of 350-400 cd/ $/ \mathrm{m}^{2}$. Furthermore, the Ti encapsulated devices show very high voltage responses to an input current density of $20 \mathrm{~mA} / \mathrm{cm}^{2}$. Worst voltage response is seen for the $5 \mathrm{~nm}$ cathode at $21.96 \mathrm{~V}$, and the best voltage response is seen for the $100 \mathrm{~nm}$ cathode at $13.5 \mathrm{~V}$. For comparison, GRP_1 OLEDs show a post-fabrication voltage response of 8-10V. These results reveal that Ti deposited via e-beam penetrates through thinner cathode layer, diminishing the device performance. 
Table 5.1 Average brightness and voltage measurements for OLEDs with a varying cathode thicknesses. The titanium layer thickness is maintained at 200nm.

\begin{tabular}{|c|c|c|}
\hline $\begin{array}{c}\text { Mg:Ag } \\
\text { thickness(nm) }\end{array}$ & $\begin{array}{c}\text { Average } \\
\text { Luminance }\left(\mathrm{cd} / \mathrm{m}^{2}\right)\end{array}$ & $\begin{array}{c}\text { Average } \\
\text { Voltage(V) }\end{array}$ \\
\hline 5 & 158 & 21.96 \\
\hline 10 & 193 & 20.03 \\
\hline 25 & 220 & 18.41 \\
\hline 50 & 296 & 16.44 \\
\hline 100 & 321 & 13.5 \\
\hline
\end{tabular}

Hence a thick cathode layer is required so as to shield the device from any deleterious effects seen as a result of Ti e-beam deposition. For this purpose a series of OLEDs are fabricated with 100nm Mg: Ag cathode and varying thicknesses of the Ti encapsulation layer. The Ti layer thickness is varied across five OLEDs with thickness values of $0,1,10,50,100 \mathrm{~nm}$. Table 5.2 shows brightness and voltage measurements for these five OLEDs, recorded postfabrication in a nitrogen environment. As can be seen from the table, increasing the Ti thickness from $10 \mathrm{~nm}$ to $50 \mathrm{~nm}$ results in non-functioning devices in spite of the 100nm Mg:Ag cathode. These findings suggest that if $\mathrm{Ti}$ is to be used as an encapsulation layer, a thick (>100nm) buffer or sacrificial layer needs to be deposited on top of the cathode before Ti deposition, to protect the OLED from Ti diffusion.

Table 5.2 Average brightness and voltage measurements for OLEDs with a varying titanium thicknesses. The Mg:Ag cathode layer thickness is maintained at 100nm.

\begin{tabular}{|c|c|c|}
\hline Ti thickness(nm) & $\begin{array}{c}\text { Average } \\
\text { Luminance }\left(\mathrm{cd} / \mathrm{m}^{2}\right)\end{array}$ & Average Voltage(V) \\
\hline 0 & 447 & 8.64 \\
\hline 1 & 483 & 8.9 \\
\hline 10 & 535 & 9.6 \\
\hline 50 & not functional & not functional \\
\hline 100 & not functional & not functional \\
\hline
\end{tabular}


To further investigate the need for buffer layer under $\mathrm{Ti}, \mathrm{MoO}_{3}$ is chosen as possible buffer layer material. $\mathrm{MoO}_{3}$ is chosen due to its good film forming qualities, and also due to the fact that it can be deposited via thermal evaporation, thereby avoiding any electron-beam induced device damage. OLEDs with the following device structure are fabricated: NPB(70nm)/ $\mathrm{Alq}_{3}(70 \mathrm{~nm}) / \mathrm{Mg}: \mathrm{Ag}(100 \mathrm{~nm}) / \mathrm{Ag}(100 \mathrm{~nm}) / \mathrm{MoO} 3(100 \mathrm{~nm}) / \mathrm{Ti}(100 \mathrm{~nm})$.

Post fabrication measurements reveal average brightness values of $415 \mathrm{~cd} / \mathrm{m}^{2}$ with an average voltage response of $8.56 \mathrm{~V}$ (for a current density of $20 \mathrm{~mA} / \mathrm{cm}^{2}$ ) for the $\mathrm{MoO} 3 / \mathrm{Ti}$ encapsulated devices. In comparison, unencapsulated OLEDs show comparable brightness and voltage values of $410 \mathrm{~cd} / \mathrm{m}^{2}$ and $8.24 \mathrm{~V}$ respectively. This observation suggests that the $\mathrm{MoO}_{3}$ buffer layer may inhibit initial Ti diffusion into the OLED, thereby improving initial performance. However, 27 hours of $100 \% \mathrm{RH}$ exposure for $\mathrm{MoO}_{3} / \mathrm{Ti}$ encapsulated OLEDs is shown to result in compromised emissive area ( $<1 \%$ functional emissive area). Even though $\mathrm{MoO} 3$ provides initial protection from Ti diffusion, MoO3/Ti layer might not help in the overall protection of the

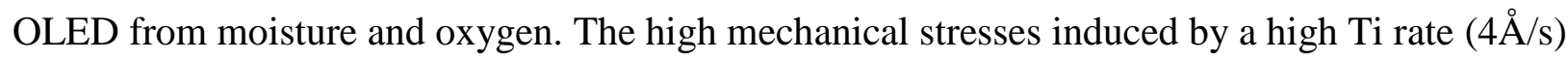
via electron beam deposition may lead to irregular Ti deposition and Ti diffusion into the OLED. Even with the use of a buffer layer, Ti may be diffusing into the organic layers - compromising the cathode-organic interface which leads to rapid dark spot growth.

From the above findings, it can be concluded that $\mathrm{Ti}$ is not a suitable choice for a OLED encapsulation layer without the use of a buffer layer to mitigate possible Ti diffusion into the OLED. In addition, less-disruptive and higher density deposition techniques like ion-beam assisted deposition need to be explored for possible Ti encapsulation strategies. 


\section{Chapter 6 Conclusions and Future Work}

\subsection{Conclusions}

Overall, it can be summarized that interface adhesion between cathode and organic layers is strengthened by heating the substrate while depositing the cathode and also by using an interfacial MOML between the cathode and the organic layers. The effect of modifying the cathode-organic interface via substrate heating or via the use of a MOML results in interfacial adhesion improvements by a factor of 2.0-2.5 on average, when compared to regular room temperature non-MOML OLEDs. Better adhesion between the cathode and organic layer via the aforementioned methods, leads to a marked retardation in the growth of dark spots in the OLED, as stronger interfacial adhesion makes cathode delamination less facile. It is interesting to note that heating the substrate while cathode deposition leads to marginally stronger cathode-organic interfacial adhesion when compared to post-deposition annealed devices(adhesion is stronger in the substrate heated cathode devices by a factor of 1.14). It is also important to note that not all interfacial layers result in increased interfacial adhesion. Structures with ultra-thin electron injection layers, such as $1 \mathrm{~nm}$ films of $\mathrm{LiF}$ or $\mathrm{Cs}_{2} \mathrm{CO}_{3}$, do not show a marked increase in interfacial adhesion.

Both of the techniques: substrate heating during cathode deposition, and MOML deposition are easily incorporated in the fabrication of an OLED and do not introduce any noticeable degree of difficulty . Both cathode deposition at elevated substrate temperatures and MOML deposition can be carried forth inside the OLED fabrication chamber itself, introducing no demanding fabrication techniques. This makes these strategies of controlling the growth of dark spots promising.

In conclusion, we have shown that the use of heating the substrate during cathode deposition as well as the use of a cathode-organic interfacial MOML is beneficial for the retardation of dark spot growth. By eliminating the roles of adsorbed residual moisture, ITO morphological changes, cathode-organic morphological changes, we speculated that increased adhesion at the cathode-organic interface may be the reason for dark spot retardation. Using a 
four-point bend tester, interfacial adhesion was shown to be stronger for elevated temperature deposited $\left(65^{\circ} \mathrm{C}\right)$ cathode OLEDs and MOML OLEDs, when compared to room temperature nonMOML OLEDs. These results indicate that increased interfacial adhesion is the reason behind dark spot suppression. Hence, by increasing cathode-organic interfacial adhesion, dark spot suppression can be achieved which can ease the need for stringent encapsulation requirements for OLEDs in today's market.

\subsection{Recommendations for Future Work}

\subsubsection{MOML -cathode transition}

As concluded in section 4.2, a MOML layer has shown to strengthen the cathode-organic interfacial adhesion. If the MOML layer can be deposited such that both the layer edges(at the ETL and the pure cathode interface) have a tapered composition, interfacial adhesion may further be improved. The effect of a completely graduated MOML layer on cathode-organic adhesion requires further investigation. A bi-directionally seamless MOML would include:

i. Gradual integration of the MOML onto the ETL surface by introducing metal onto the substrate when the ETL organic material is still being deposited. Careful control of the deposition rates is important for a smooth transition from ETL to a MOML layer. A fabrication chamber which allows for seamless metal integration into an ongoing deposition process would make this process feasible.

ii. Gradually decreasing the organic material from the MOML mixture while deposition, should result in a tapered end for the MOML and a highly graded initiation for the cathode layer. Although this step was attempted in section 4.3, the ETL rate was abruptly reduced to $0 \AA / \mathrm{s}$, possibly not achieving the desired tapered MOML effect.

\subsubsection{Adhesion Testing}

As seen in section 3.2.3, the cathode can be easily peeled off with $3 \mathrm{M}$ scotch tape. The ETL layer does not get peeled off, as the peeled device still exhibits ETL photoluminescence 
when subjected to UV radiation. Hence the cathode-organic interface was stated to be the weakest. When conducting the four point bend test, the crack propagates along the weakest interface. Due to the evidence from the cathode peel off test, we assume that the weakest interface is the cathode-organic interface. To further prove this assumption, X-ray photoelectron spectroscopy(XPS) of the fractured interface is required. The chemical composition along the fractured interface can provide details on the exact interface where the crack propagates in the bending test.

\subsubsection{Encapsulation}

Although Titanium has not proved to be an ideal encapsulant for an OLED, investigations into appropriate buffer layers to enable Titanium encapsulation are required. Electron-beam (ebeam) deposition of Titanium using a high deposition rate may have resulted in a) rougher Titanium film morphology, and b) Titanium diffusion into the organic layers in spite of a 100nm $\mathrm{MoO} 3$ buffer layer. Therefore, the following experiments need to be conducted:

i. AFM morphology scans of the Ti layer: This will help analyze the roughness of the electron-beam deposited Ti layer.

ii. XPS at the cathode-organic interface of a MoO3/Ti encapsulated OLED : This can provide details on the degree of Ti diffusion into the organic layers.

iii. Ion-Beam Assisted Deposition(IBAD) of Ti for OLED encapsulation: IBAD has the potential of forming high density films nearly devoid of pinholes which may aid in forming high integrity encapsulation structures for OLEDs. 


\section{References}

1 Koninklijke Philips Electronics, Philips Lumiblade Shop from https://www. lumibladeshop.com/index.php/

2 Koninklijke Philips Electronics, Philips Lighting from http://www.lighting.philips.cal

3 Sony, OLED TV XEL-1 from http:// store.sony.com/

4 Fraunhofer ISE, Organic Solar Cell from http://www.sciencedaily.com/releases/2008/02/080206154631.htm

5 Pacific Northwest National Laboratory, US Department of Energy, Blue Light Specials from http://www.pnl.gov/news/release.aspx?id=358

6 World of Photovoltaics, The Promise of Organic Electronics from http://www.worldofphotovoltaics.com/equipment_and_materials/substrates/the_promise_ of_organic_electronics.html

7 C. W. Tang, S. A. VanSlyke, Appl. Phys. Lett. 51, 913 (1987).

8 W. Helfrich, W. G. Schneider, Phys. Rev. Lett. 14, 229 (1965).

9 S. Schrader, Organic Photonic Materials and Devices V 4991, 45 (2003).

10 P. L. Burn, A. B. Holmes, A. Kraft, A. R. Brown, D. D. C. Bradley and R. H. Friend, Light-Emitting-Diodes Based on Conjugated Polymers - Control of Color and Efficiency, 1992), p. 654.

11 F. Cacialli, R. H. Friend, N. Haylett, R. Daik, W. J. Feast, D. A. dosSantos and J. L. Bredas, Appl. Phys. Lett. 69, 3794 (1996).

12 D. G. Ma, Z. Y. Hong, X. J. Zhao, X. B. Jing, F. S. Wang, J. W. Shi, M. D. Liu, E. S. Jin and S. W. Li, Chinese Physics Letters 13, 940 (1996).

13 H. Antoniadis, D. Roitman, B. Hsieh and W. A. Feld, Polym. Adv. Technol. 8, 392 (1997).

14 W. Riess, Polym. Adv. Technol. 8, 381 (1997).

15 S. Schrader, W. Riess, H. Vestweber and N. Koch, Organic Light-Emitting Materials and Devices Ii 3476, 188 (1998).

16 W. Li, J. E. Malinsky, H. Chou, W. Ma, L. Geng, T. J. Marks, G. E. Jabbour, S. E. Shaheen, B. Kippelen, N. Peyghambarian, J. Anderson, P. Lee and N. Armstrong, 
Electrical, Optical, and Magnetic Properties of Organic Solid-State Materials Iv 488, 385 (1998).

17 D. O'Brien, A. Bleyer, D. G. Lidzey, D. D. C. Bradley and T. Tsutsui, Optical Materials 9, 173 (1998).

18 L. C. Picciolo, H. Murata and Z. H. Kafafi, Appl. Phys. Lett. 78, 2378 (2001).

19 University of Washington, Organic Light Emitting Diodes from http://depts.washington.edu/cmditr/modules/oled/organicorganic_heterojunctions_in_ole ds.html

20 Wikipedia, Organic Light Emitting Diodes from http://en.wikipedia.org/wiki/Organic_light-emitting_diode

21 W. Schottky, Phys. Z. 41, 570 (1940).

22 Rensselaer Polytechnic Institute, ECE 2210 course tutorial.

23 H. Ishii, K. Sugiyama, E. Ito, and K. Seki, Adv. Mater. 11, 605 (1999).

24 University of Waterloo, ECE730 Class Tutorial- Winter 2009 -Prof. H. Aziz.

25 W. Brutting, ICEL2 Tutorial on OLEDs.

26 W. Y. Liang 1970 Phys. Educ. 5226.

27 Israel Institue of Technology-Technion, Electrical Engineering Course Material from http://webee.technion.ac.il/orgelect/excitons.pdf

28 H. Aziz and ZD Popovic, Chem. Mat. 16, 4522 (2004).

29 Z. D. Popovic, H. Aziz, N. X. Hu, A. M. Hor and G. Xu, Organic Light-Emitting Materials and Devices Iii 3797, 310 (1999).

30 J. McElvain, H. Antoniadis, M. R. Hueschen, J. N. Miller, D. M. Roitman, J. R. Sheats and R. L. Moon, J. Appl. Phys. 80, 6002 (1996).

31 S. F. Lim, L. Ke, W. Wang and S. J. Chua, Appl. Phys. Lett. 78, 2116 (2001).

32 V.N. Savvateev, A.H. Yakimov,D. Davidov, R.M. Pogreb, R. Neumann, Y. Avny, Appl. Phys. Lett., 71, 3344(1997).

33 L.M. Do, K. Kim,T. Zyung, H.K. Shim, J.J. Kim, Appl. Phys. Lett. 70, 3470(1997)

34 M. Kawaharada, M. Ooishi, T.Saito, E. Hasegawa, Synth. Met. 91,113(1997).

35 H. Aziz, Z. Popovic, C. P. Tripp, N. X. Hu, A. M. Hor and G. Xu, Appl. Phys. Lett. 72, 2642 (1998). 
36 L. S. Liao, J. He, X. Zhou, M. Lu, Z.H. Xiong, Z.B. Deng, X.Y. Hou, S.T. Lee, J. Appl. Phys. 88, 2386(2000).

37 M. Schaer, F. Nuesch, D. Berner,W. Leo, L. Zuppiroli, Adv. Funct. Mater. 11, 116(2001).

38 C. Y. Li, B. Wei and J. H. Zhang, Encapsulation of Organic Light-emitting Devices for the Application of Display,_(IEEE, New York; 345 E 47th St, New York, NY 10017 USA, 2008), p. 522.

39 H. Aziz, Z. Popovic, S. Xie, A. M. Hor, N. X. Hu, C. Tripp and G. Xu, Appl. Phys. Lett. 72, 756 (1998).

40 Z.Q. Gao, W.Y. Lai, T.C. Wong, C.S. Lee, I. Bello, S.T. Lee, Appl. Phys. Lett. 74, 3269(1999).

41 M.Y. Chan, S.L. Lai, F.L. Wong, O. Lengyel, C.S. Lee, S.T. Lee, Chem. Phys.Lett.371 ,700 (2003).

42 Z.Q. Gao, W.Y. Lai, T.C. Wong, C.S. Lee, I. Bello, S.T. Lee, Appl.Phys.Lett. 74 , 3269(1999).

43 Z.Q. Gao, W.Y. Lai, T.C. Wong, C.S. Lee, I. Bello, S.T. Lee,Appl.Phys.Lett. 77 , 3113(2000).

44 M. S. Xu and J. B. Xu, Thin Solid Films 491, 317 (2005).

45 C. Y. Kwong, A. B. Djurisic, V. L. Roy, P. Lai and W. K. Chan, Thin Solid Films 458, 281 (2004).

46 Y. F. Liew, H. Aziz, N. X. Hu, H. S. O. Chan, G. Xu and Z. Popovic, Appl. Phys. Lett. 77,2650 (2000).

47 J.S. Lewis, M.S. Weaver, IEEE J. Sel. Top. Quantum Electron. 10, 45 (2004).

48 Vitex Systems, Barix thin film encapsulation from http://www.vitexsys.com/index.html

49 A.B. Chwang, M.A. Rothman, Appl. Phys. Lett. 83 (3), 413(2003).

50 M. C. Sun, J. H. Jou, W. K. Weng and Y. S. Huang, Thin Solid Films 491, 260 (2005).

51 B. J. Chen, X. W. Sun, T. K. S. Wong, X. Hu and A. Uddin, Appl. Phys. Lett. 87, 063505 (2005).

52 F. L. Wong, H. Y. Sun, S. W. Tong, M. Y. Chan, C. S. Lee and S. T. Lee, J. Cryst. Growth 288, 110 (2006). 
53 H. Aziz, YF Liew and Z. Popovic, Organic Light-Emitting Materials and Devices Vii 5214, 277 (2004).

54 Q. Ma, J. Mater. Res. 12, 840 (1997).

55 R. Dauskardt, M. Lane, Q. Ma and N. Krishna, Eng. Fract. Mech. 61, 141 (1998).

56 C. W. Chen, T. Y. Cho, C. C. Wu, H. L. Yu and T. Y. Luh, Appl. Phys. Lett. 81, 1570 (2002).

57 M. S. Xu and J. B. Xu, J. Phys. D 37, 1603 (2004).

58 K. Koike, F. Yamazaki, T. Okamura and S. Fukuda, J. Vac. Science \& Tech. a 25, 527 (2007). 